
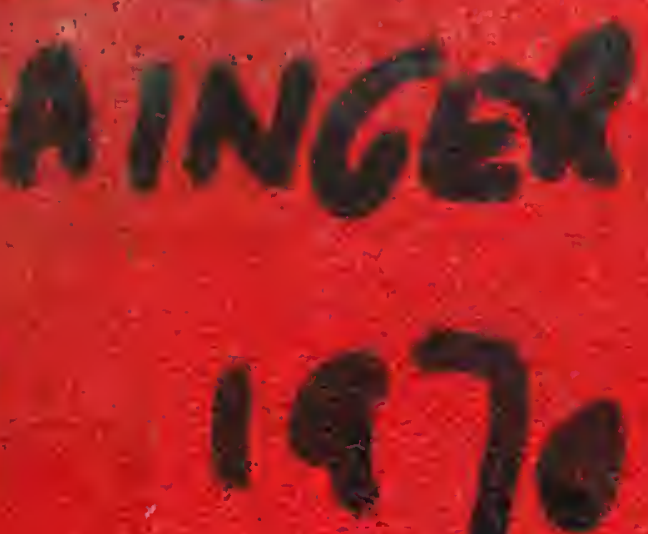

\title{
0010
}
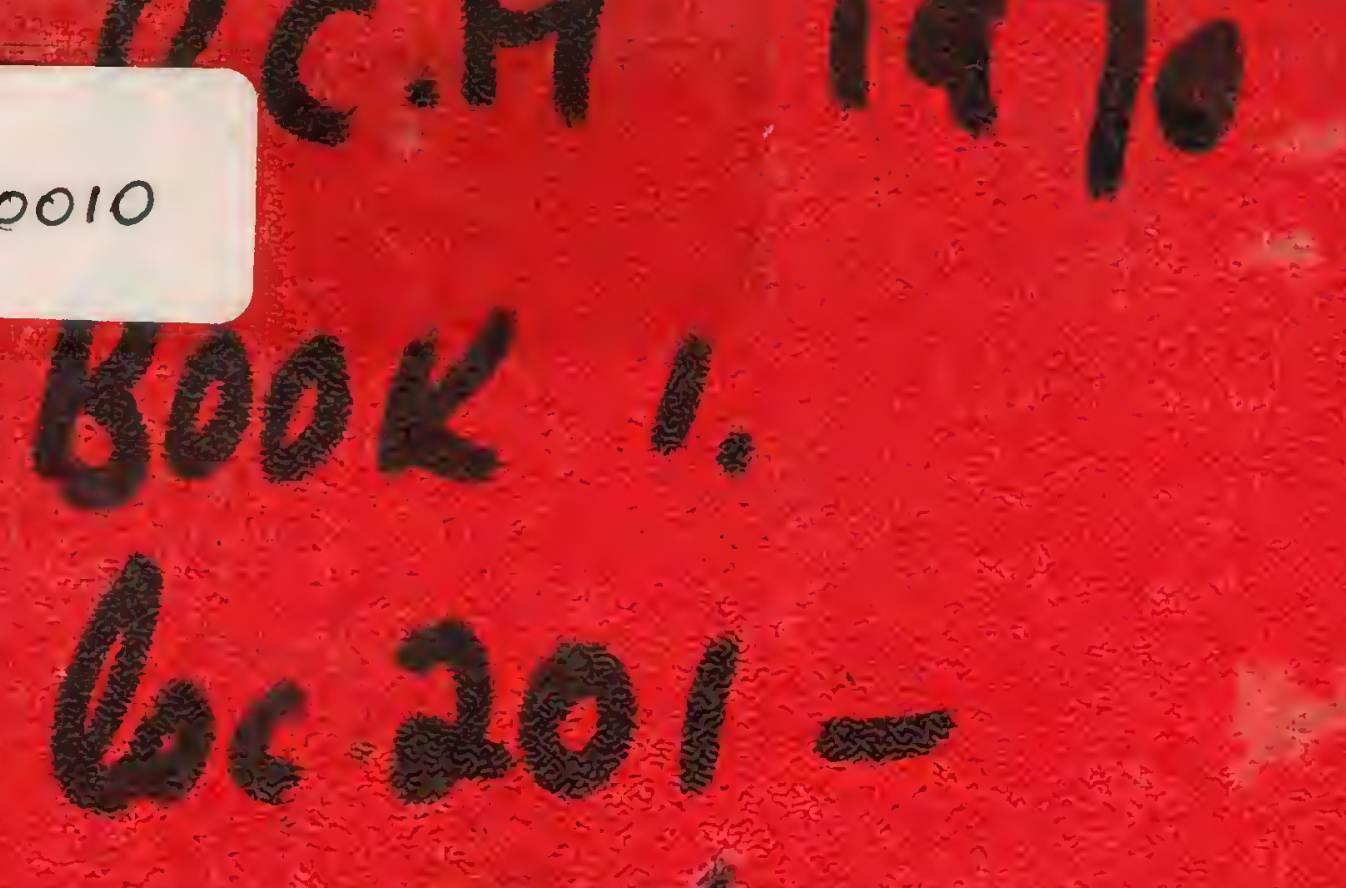

s. $x^{2}$

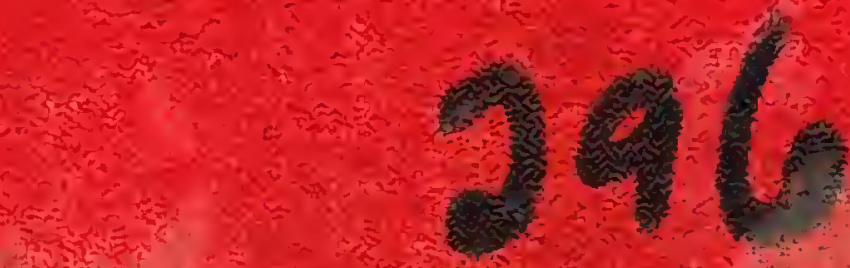

and

astion

a.

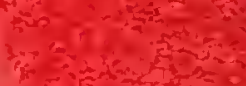

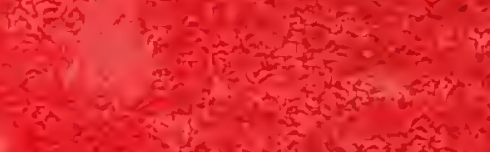
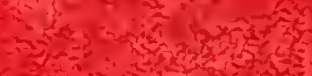

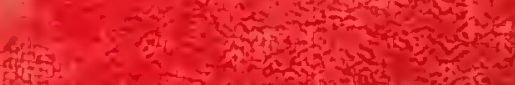


IN DEX

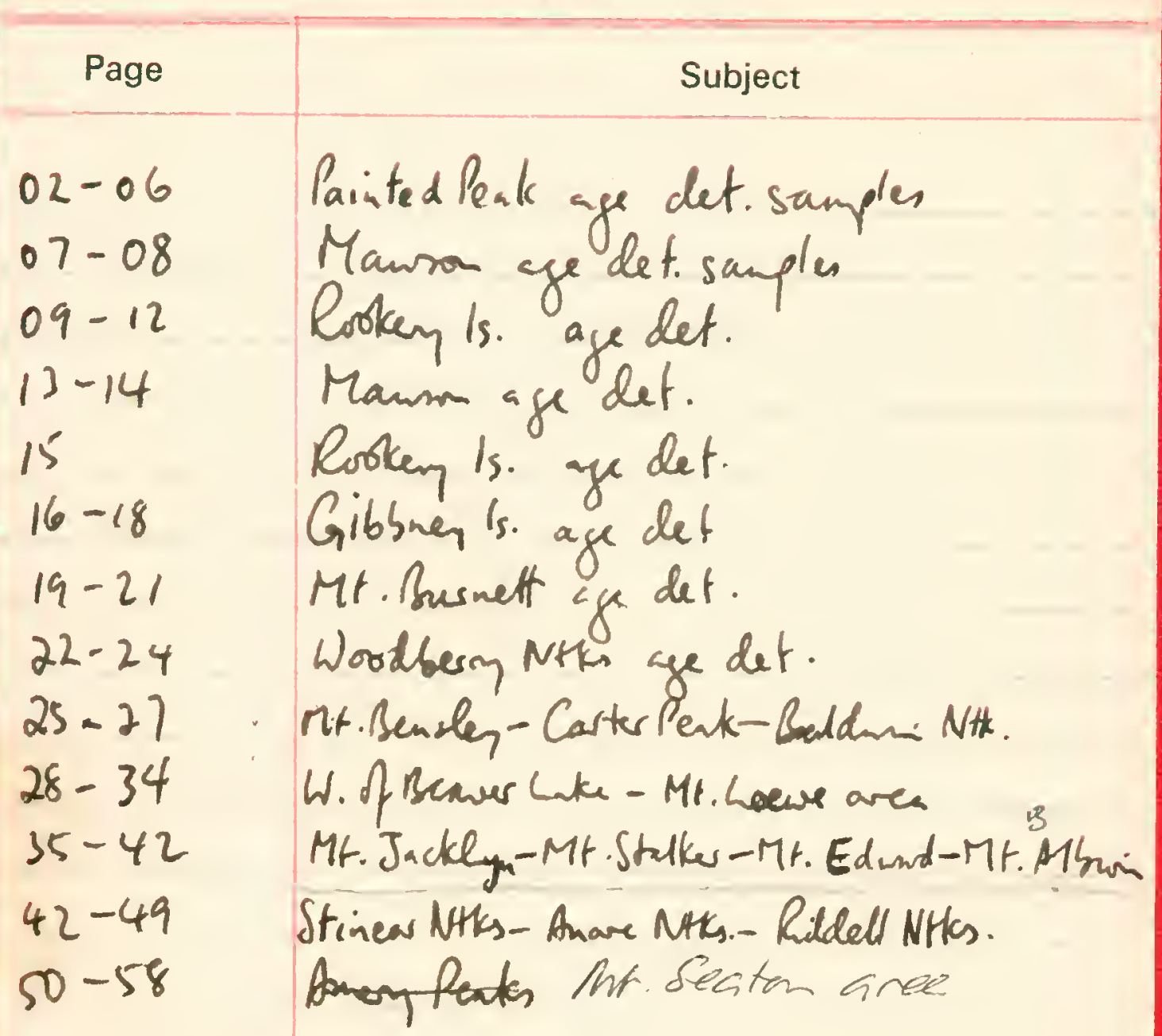


the READInGS MAgILTC

Declination about 650w-i.e Subtract $65^{\circ}$ fram masnetic readonjs 
SUNDAY $25^{\text {"W }}$ TAN 1970

Age Cextuminatix sangles

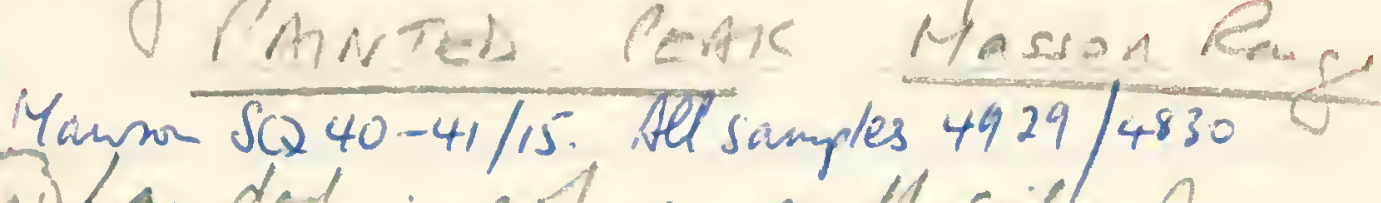

(20) Landed cat a mall ruile of Paunted Reak. Took graik redily;

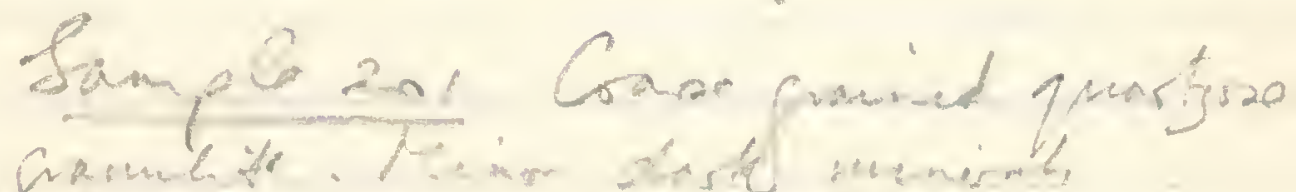

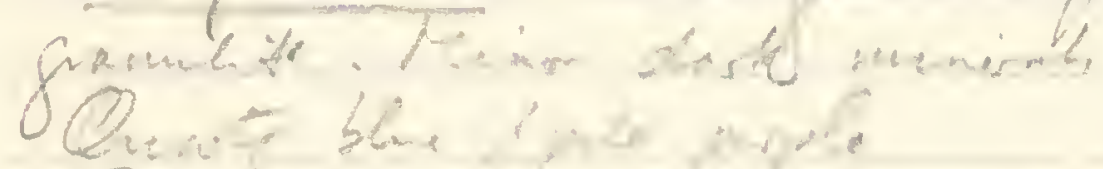

Fom lenses ad $r+2+2 m$.

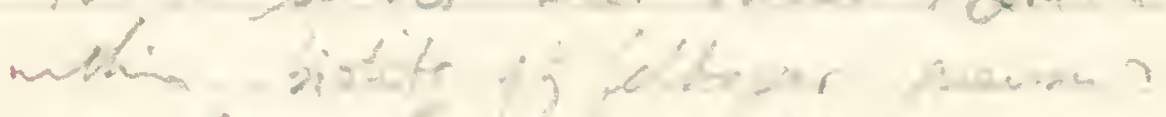

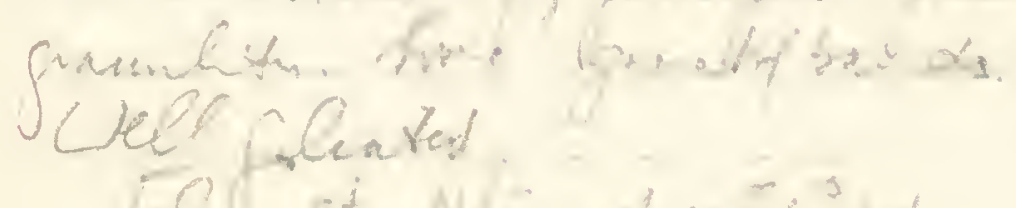

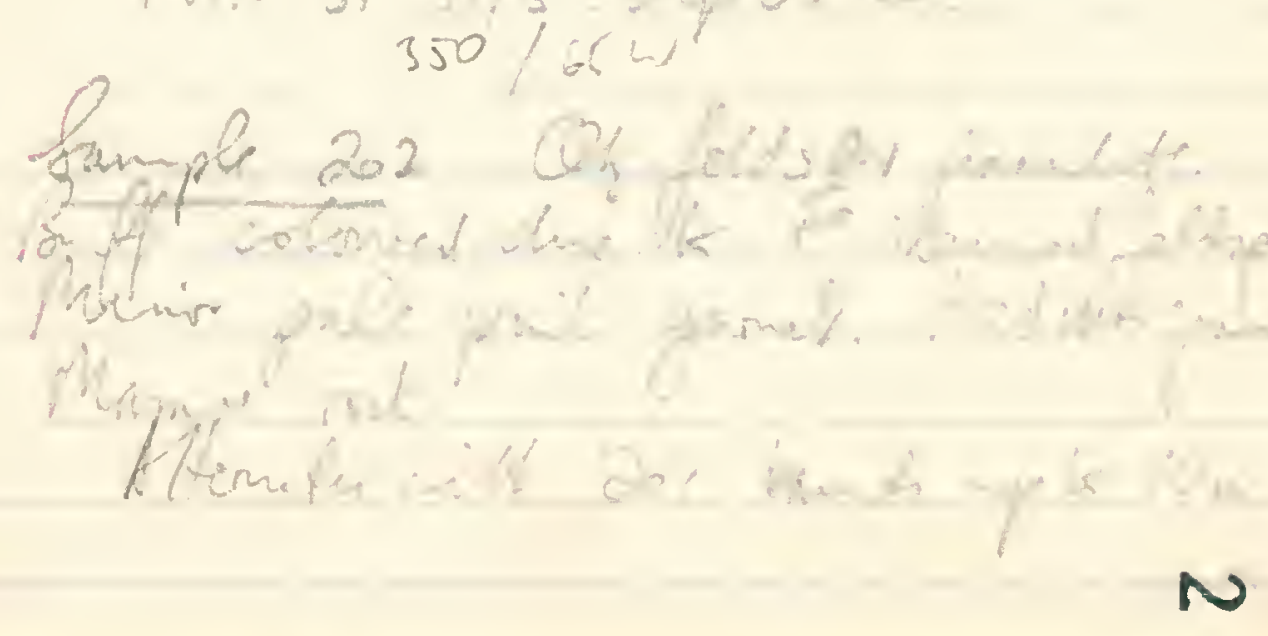


U

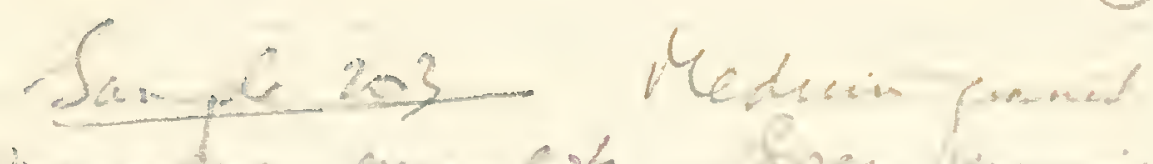

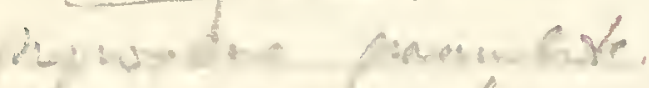

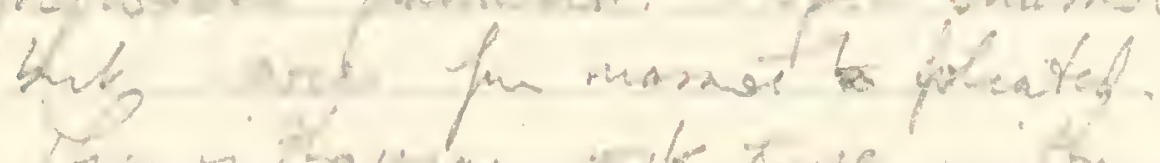

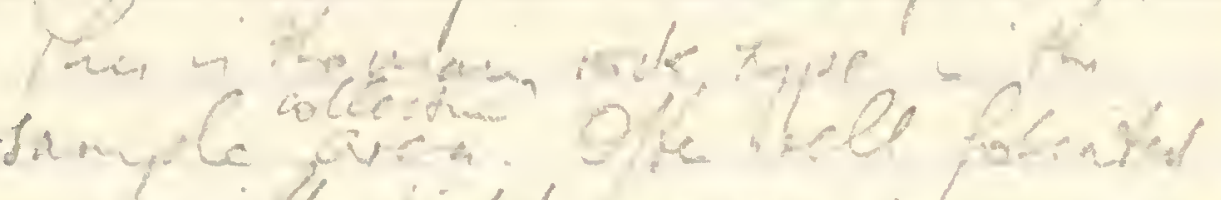

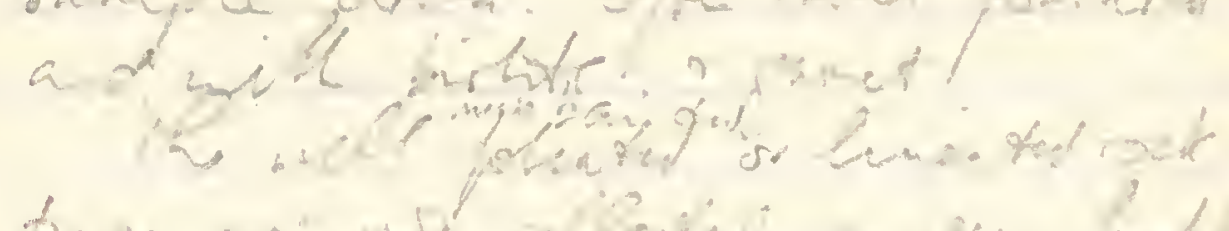

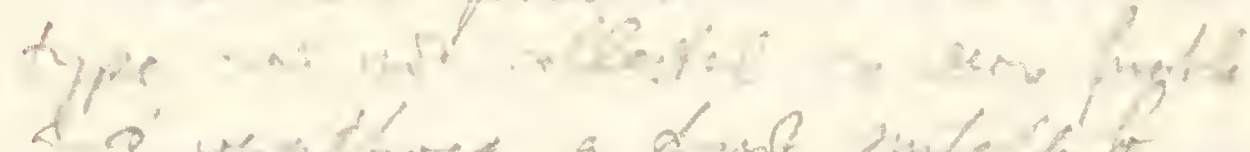

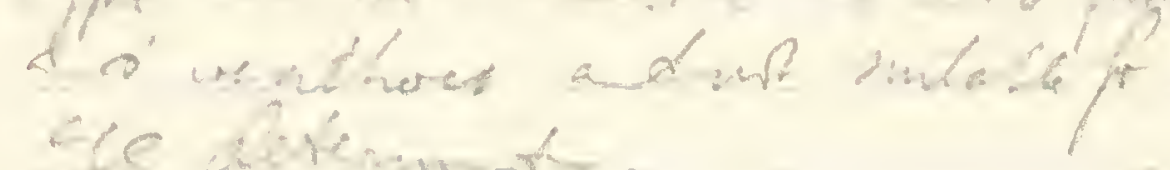
te sekinmot.

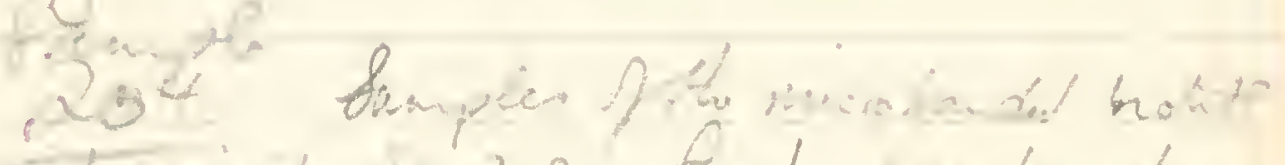

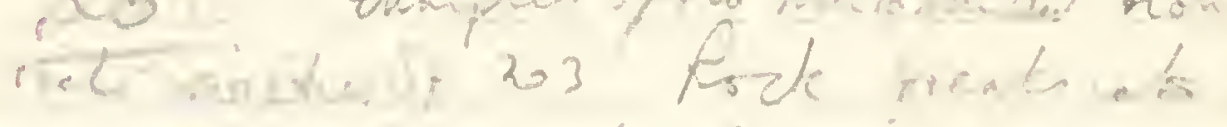

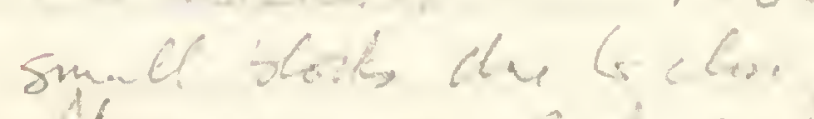

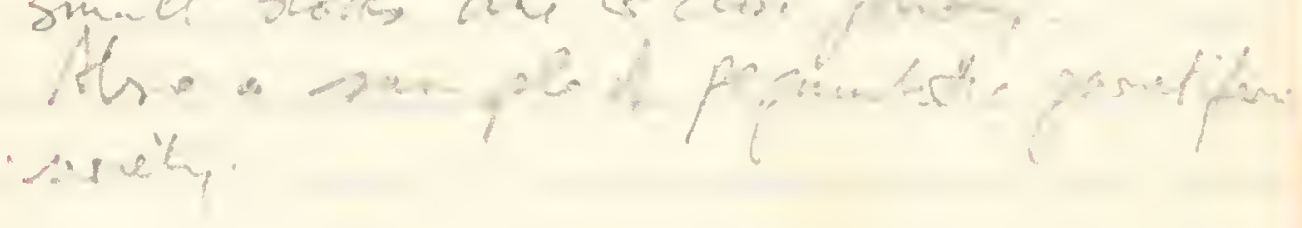

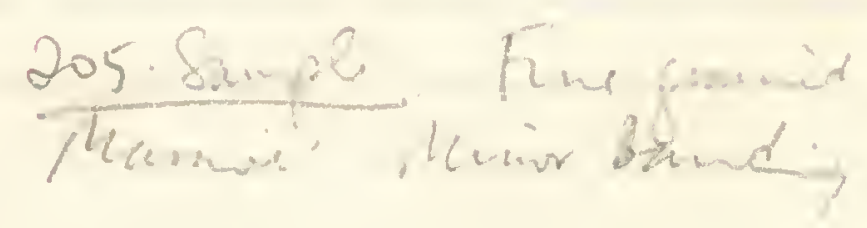


Simple 200 Calaite rick rouk

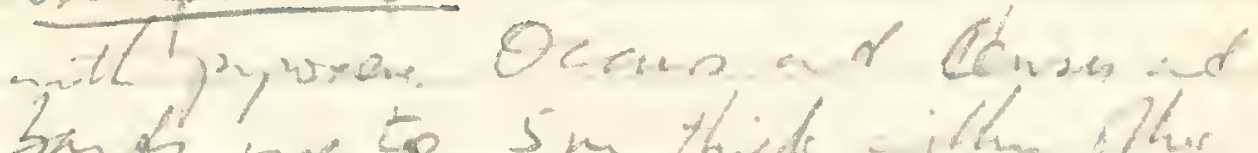

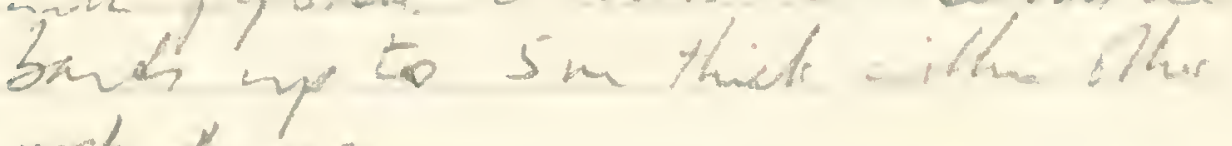
rect ofyes.

Hef asm

$\frac{\text { SArage 20? }}{\text { Camet } 9=}$

Coare ponmont matnis.

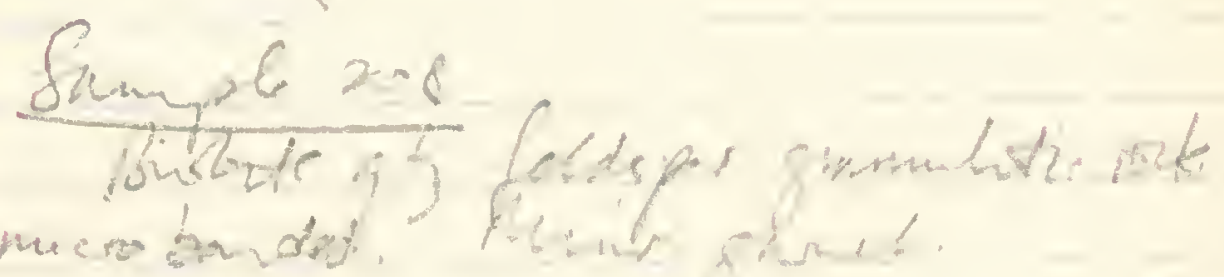
mucro bavdet.

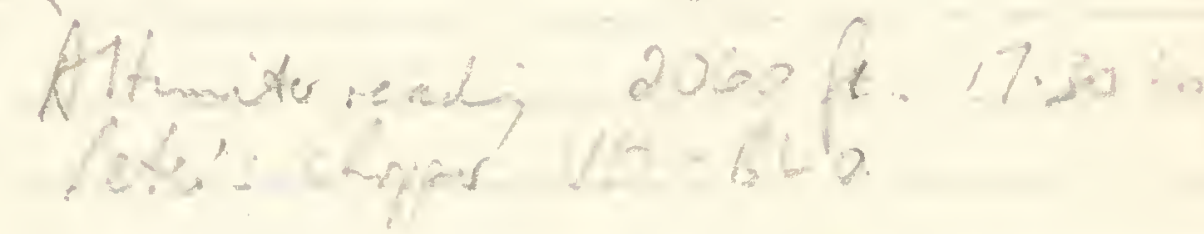

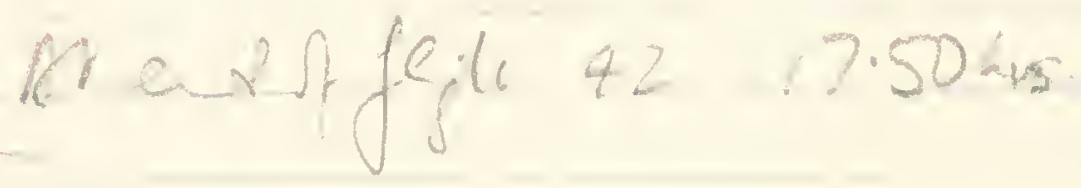


Costh a sumthing cot geverall

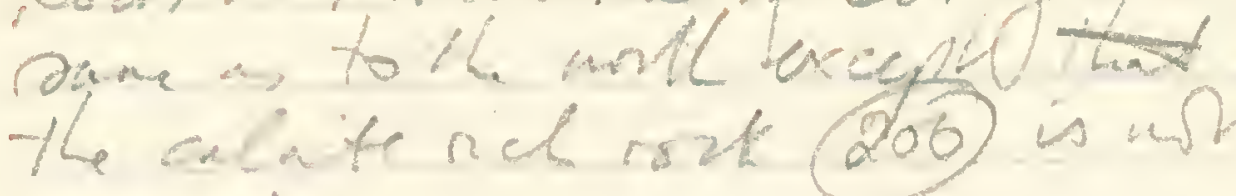
perat

Pointed Rak thant perbing

Akeration lay sanle wrotur A gimmes meduim is dane

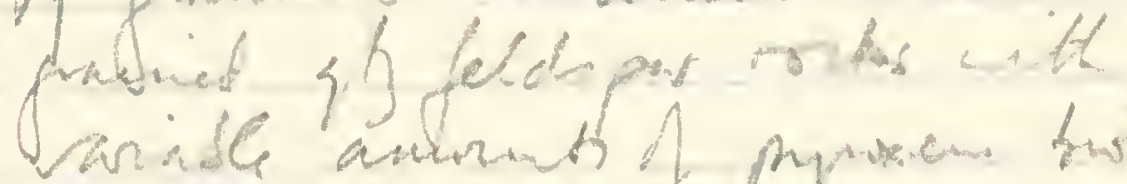

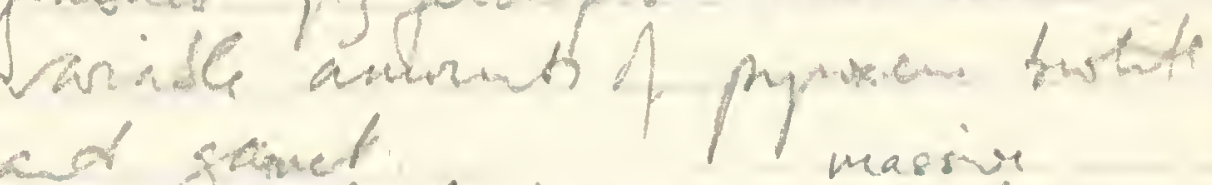
ad gamed massive

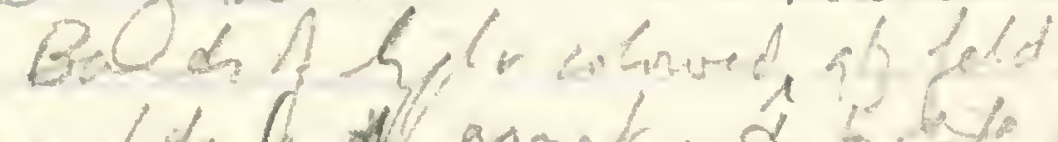
gromble (widh garet ad bute.

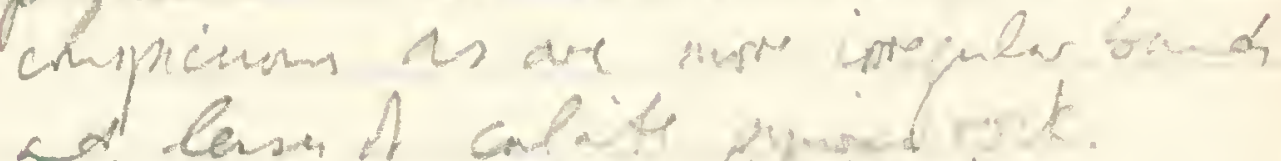

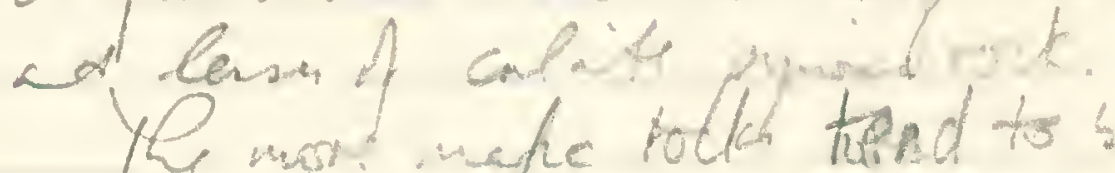
The mor. mafe rock tend to be micobsonded wown themselere ox there

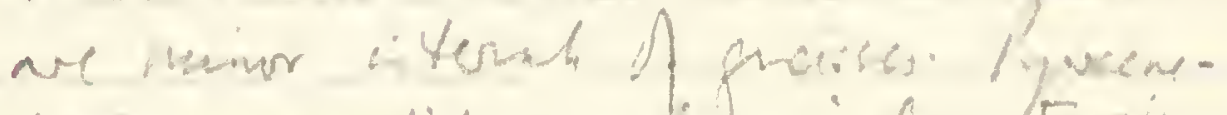
biskte granulitas pouldurinate. Fes they do fridle ad weel

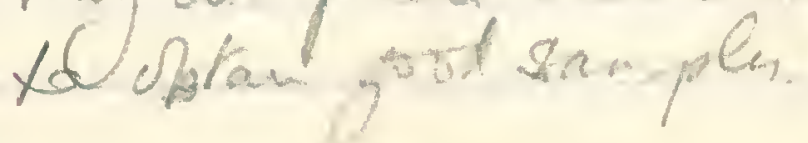


(4)

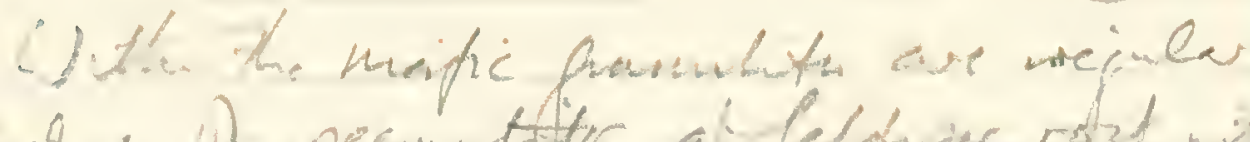

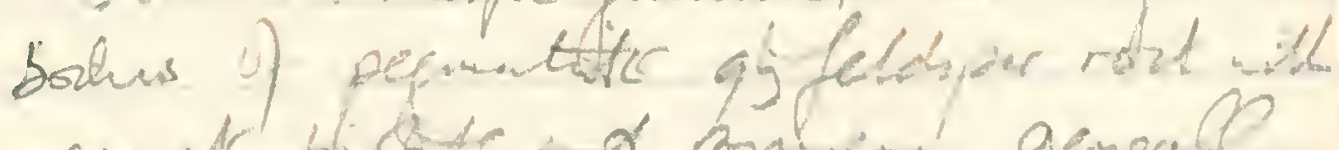

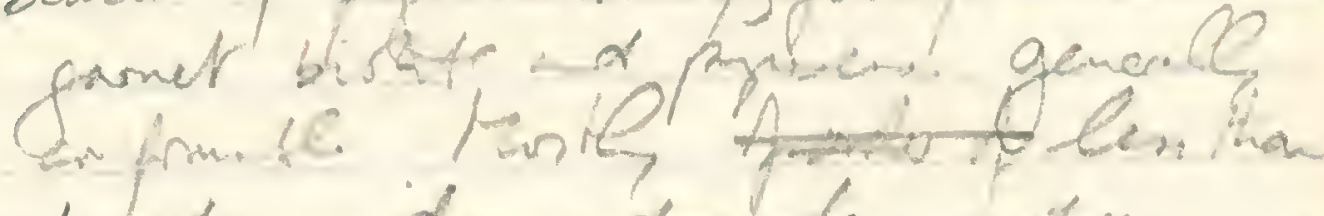
1 hetar wide ad. fow metres. $\log t$. 
Yollity 26 JARUARY 1970

5)

MnISoiv Rock colleotin If wichum Manma $\delta Q 40-41 / 15$. Samples les:4947/5011 Lozetion 20 ?

If hoist in bill reer Hectemolspad

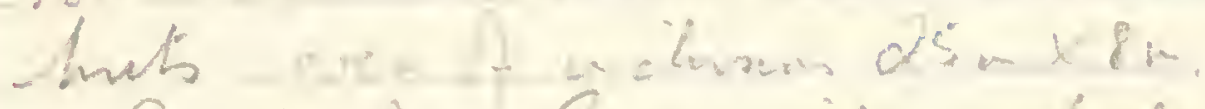

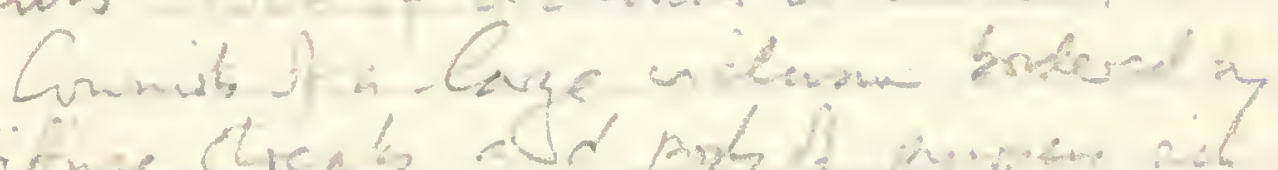

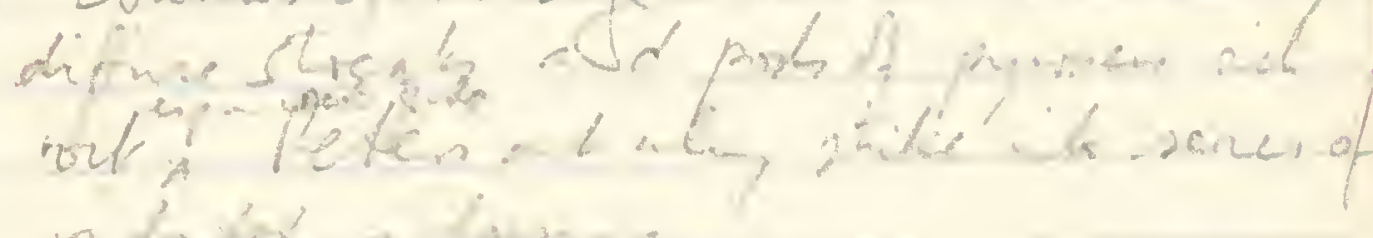

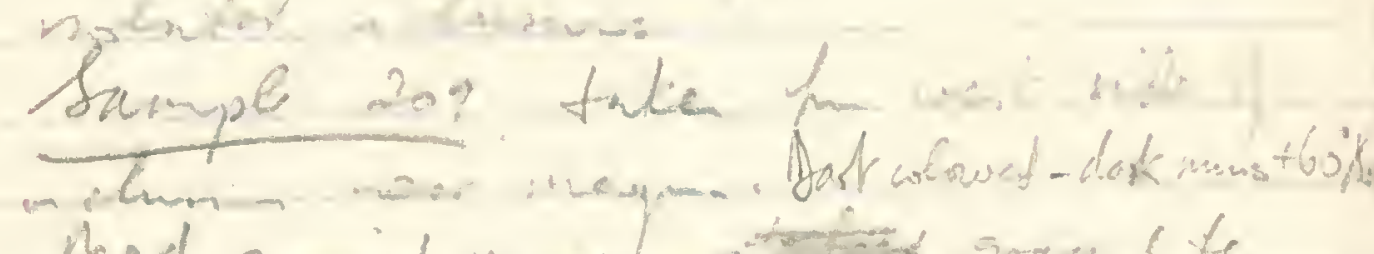

alenc.

twoter proven nit jouks

- Lample hete wive

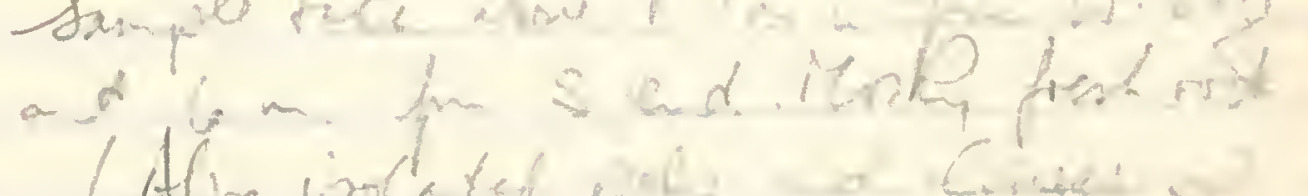

( Alw inde fef 
Tainge 210

(6)

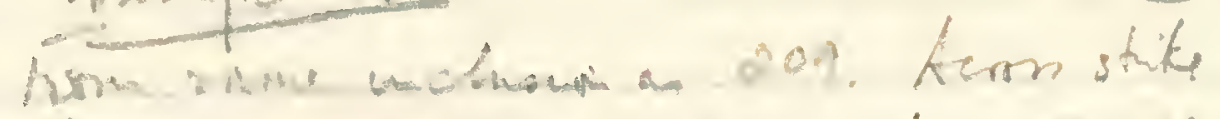

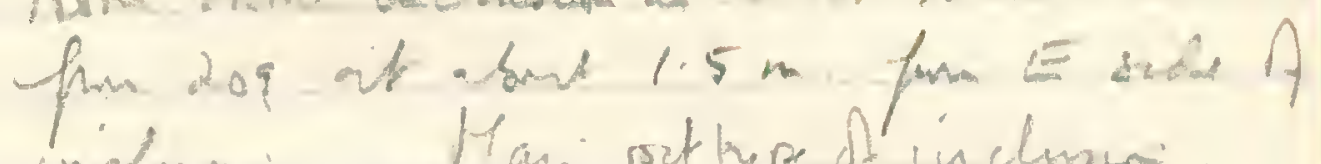

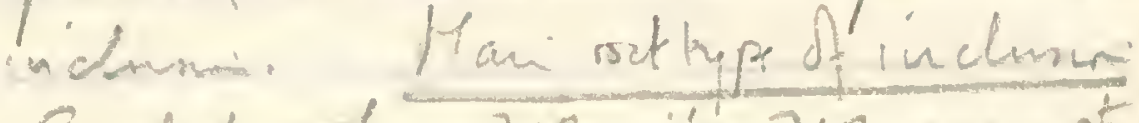
Gralath for 209 - ita 210 acerristike.

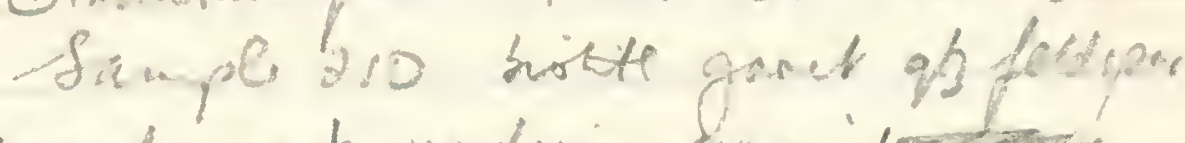
gramblas rock we devein frumin Gamets pale paik $\mathrm{CO}_{3}$ blue grad

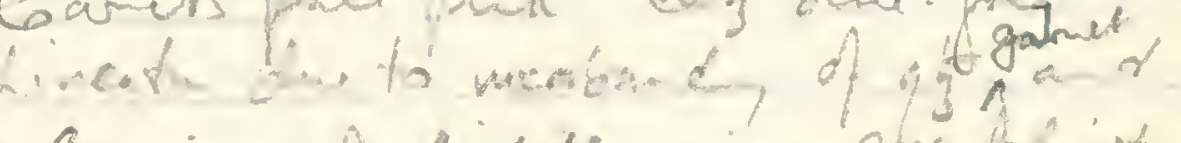

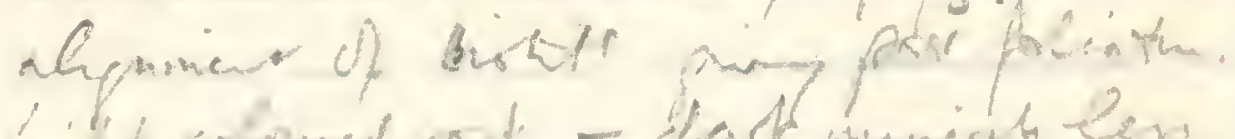

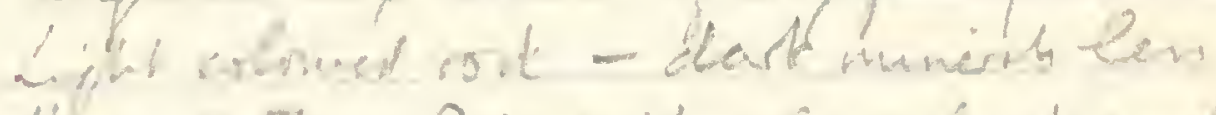

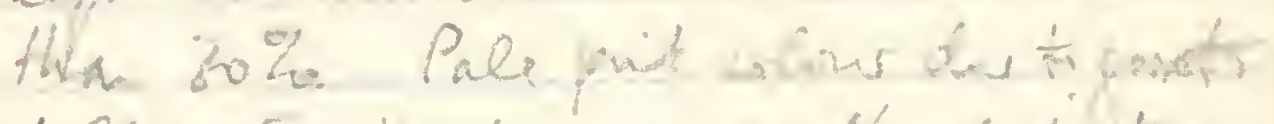
Yelen is stasin; a weathered frots Romed unify form

- 43 Fista. If ramplezpo

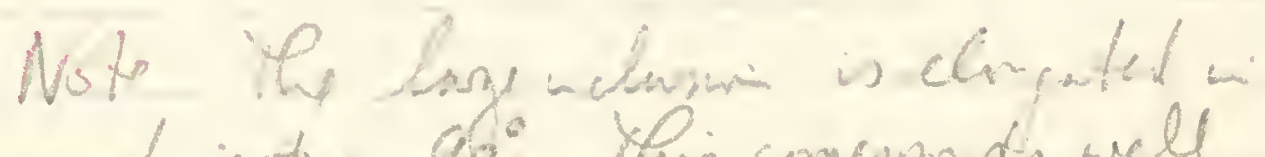
a durecto $99^{\circ}$ this comerpmeds well ath the fenerd stite of the inclusmin 2 th overof the Het.lants viz75-95.

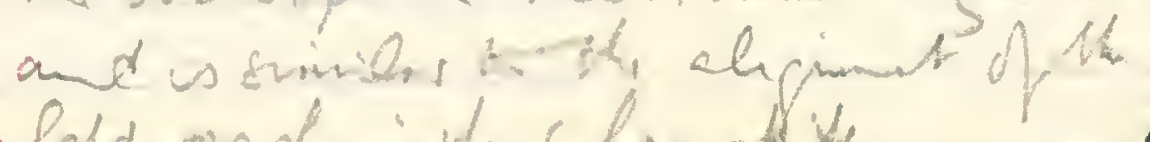
fedt puphos in chomontite. $\infty$ 
MONOM 26 J JANUARY 970

(7)

Rookom islands Aze let. hocath (211) Maum SQ240-41/15. Ref.4788/5001

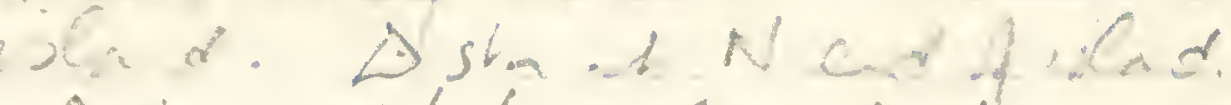
$\Delta \sin . N M / 5 / 60 \quad 62 m$. eleratur Simpa 211

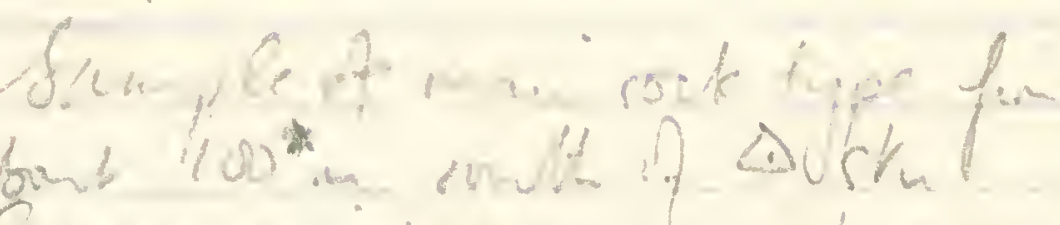

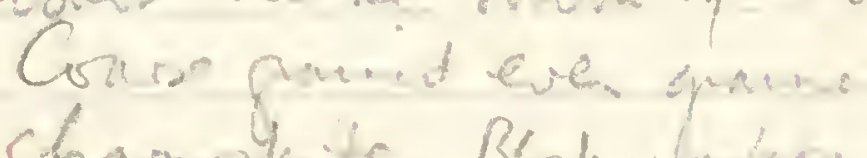

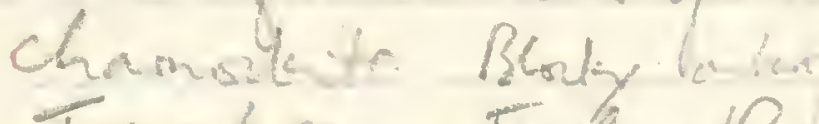

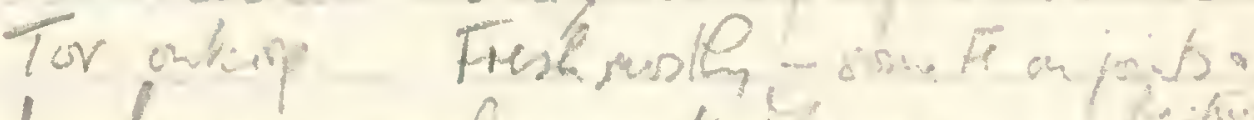

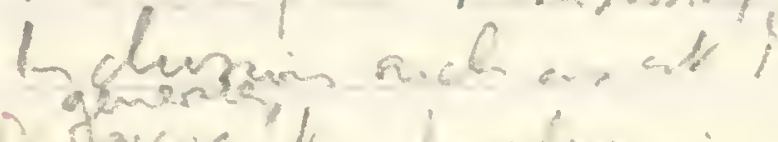

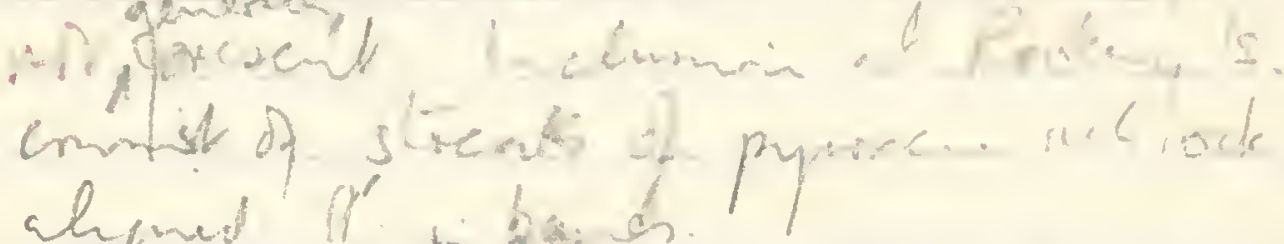
ahpout to ba: on

$\operatorname{ton}_{\sin }^{600}$ 
$\sin x^{2} 212$

(8)

$$
75 n \text { SK L S S m. }
$$

rest sample

sumear $f$

sel.

Pyase

Simple 213

$50 \mathrm{~m}$ SE Jum OSt

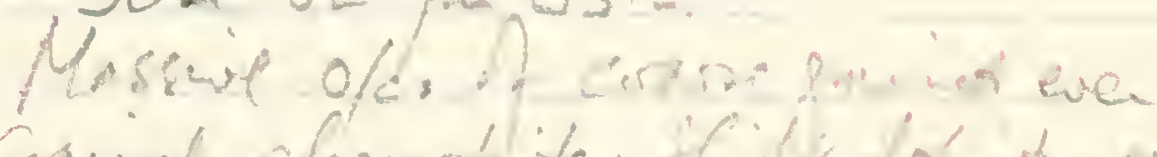

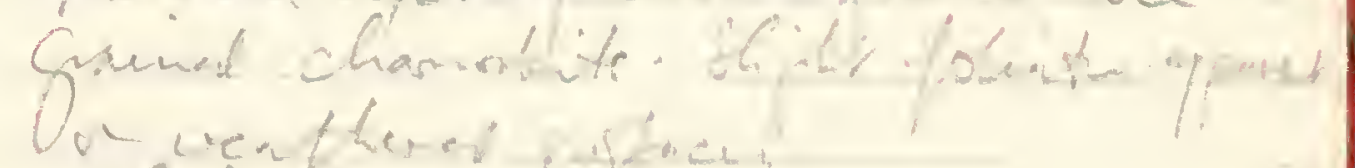

ov wenther

treese

Basterested 3

All simles -

$214,215,210$

$\vec{\circ}$ 
(9)

Gencel acolong if Rooker ISlans

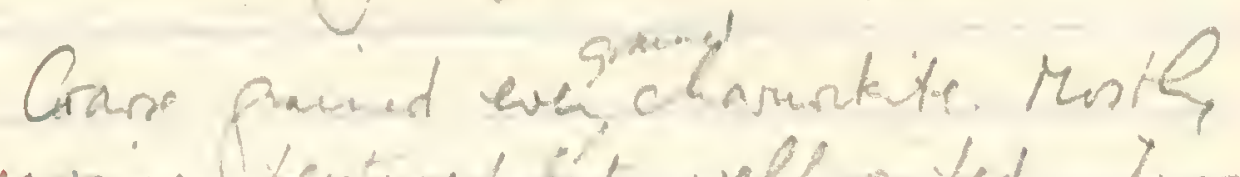

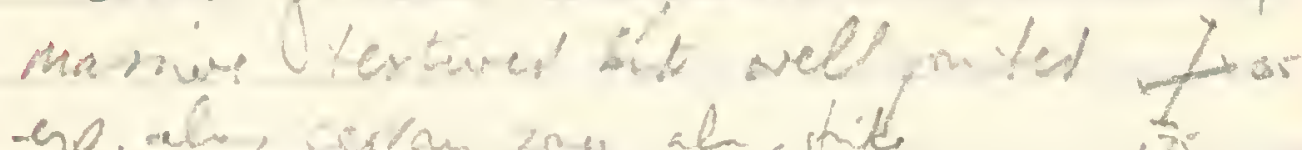
-ap. ale cerrom zone, al, stide.

- Cleyer kigh mits

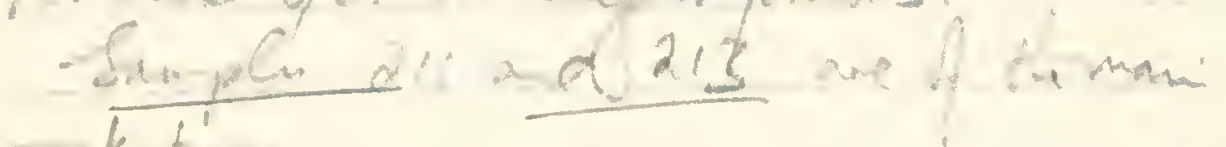

stiphe.

in place a feration in unpital

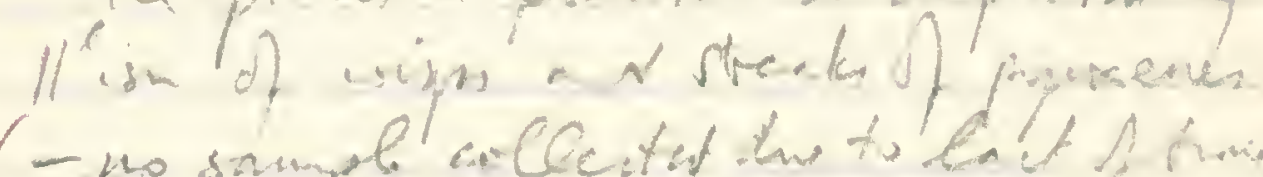
(-no scumple allectut ine to bat f

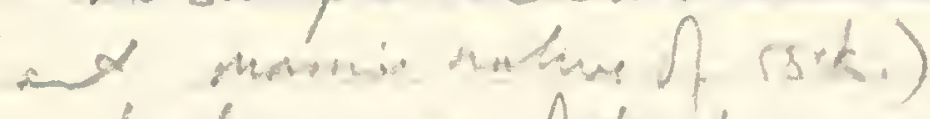

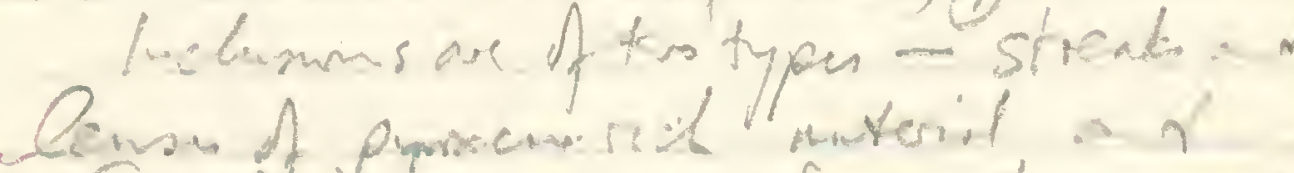
Censen of proment rod materid, an

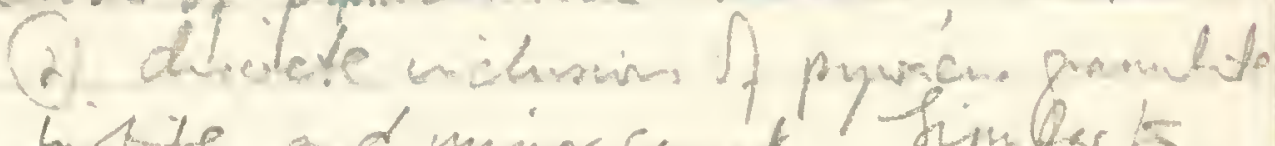
+ buste ad minr camet. Jimbark

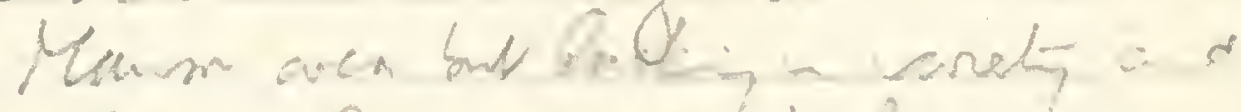

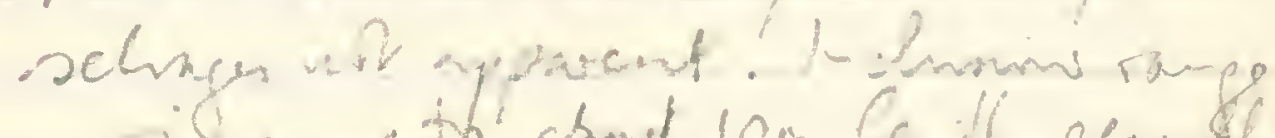

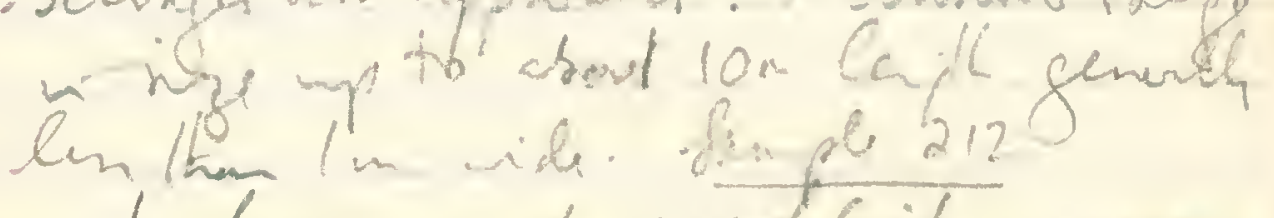

Inclumis and poss plavitu mote troo 


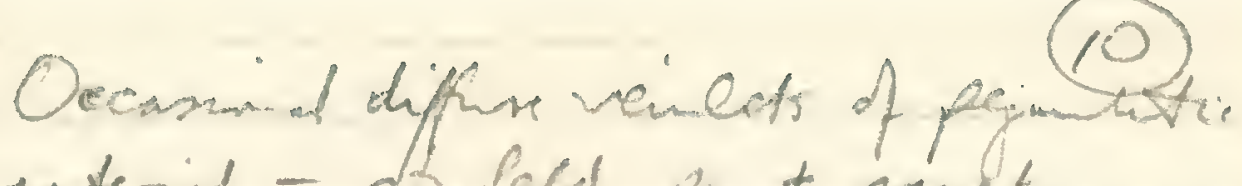
material - of feld, 12 , I gavet.

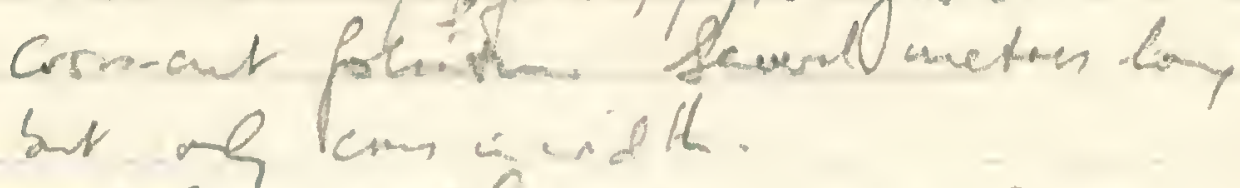

Note no the ave zous were rated of irawom aren

Bramobite - I Rorkey 12 les much mare struphy fisinted than at Karm.

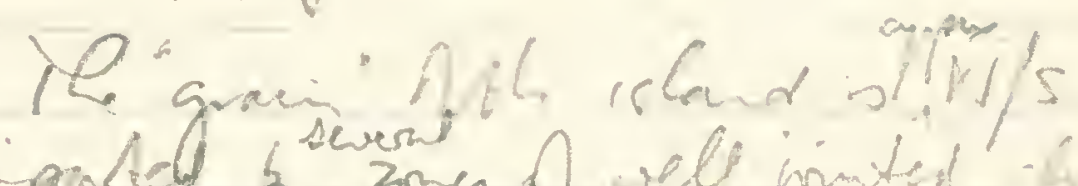

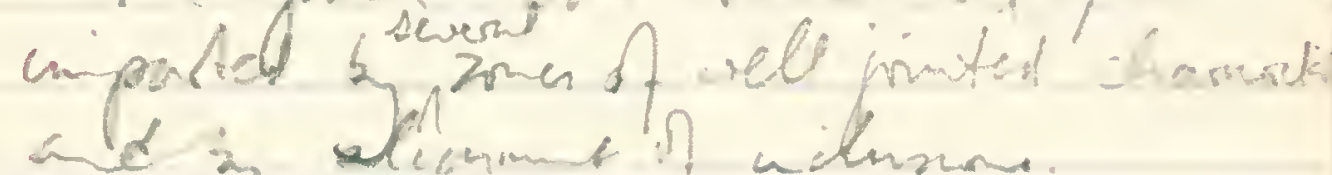
ad as slifame? willonos.

Fored to return to Masron afir lewthom on how becsense of inceconic cimir speed.

Poskey island - many pengivins wist pory. Lage aren If guano.

$\vec{N}$ 
Miowson Rock $28^{\text {th }}$ 殒 1970

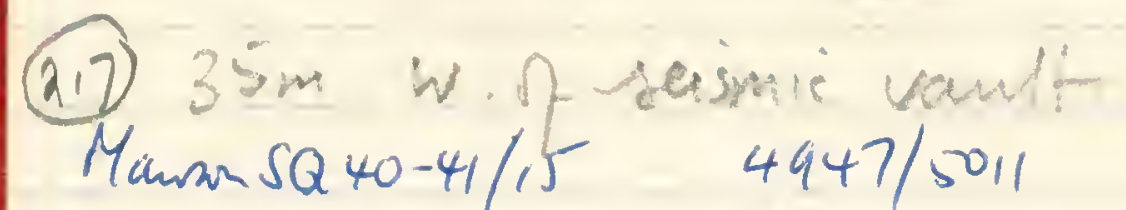
Induare 1 in lon $\times 10 \mathrm{cmande}$ elngated 5 .5 Fin - mat promed

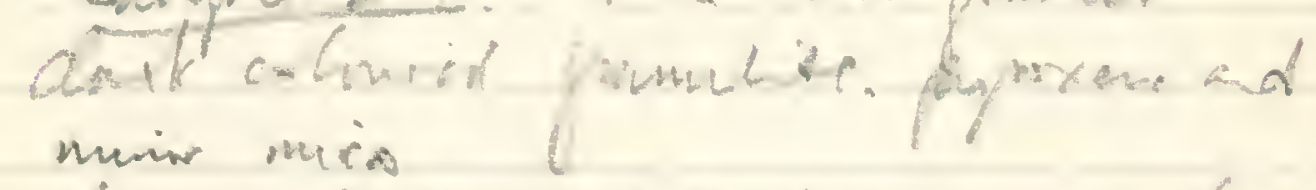

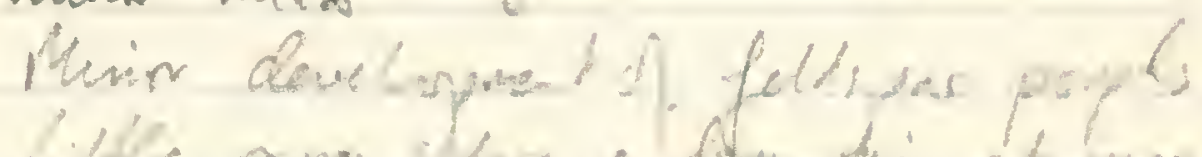

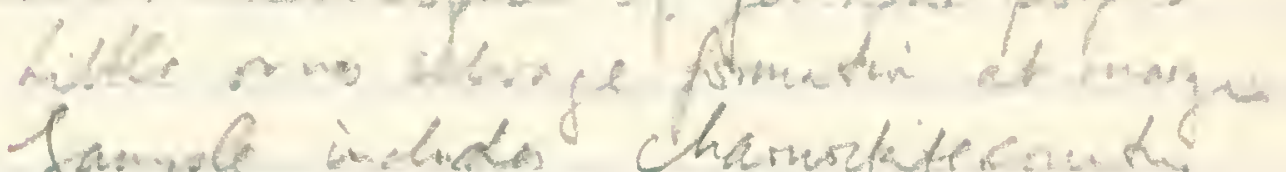

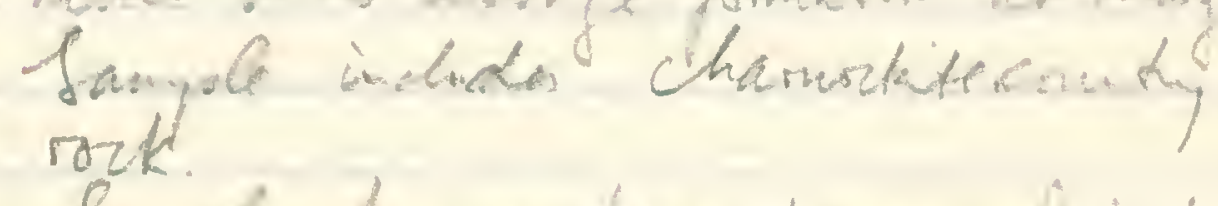

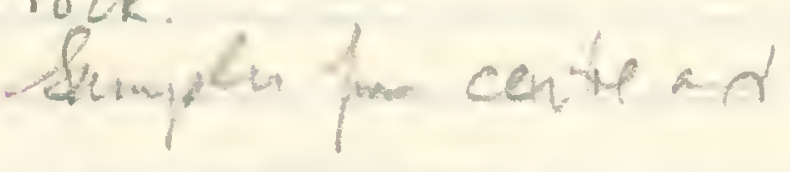

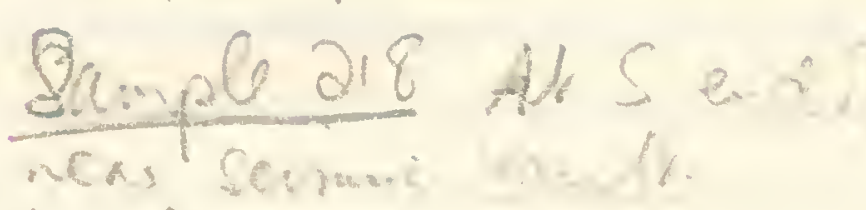

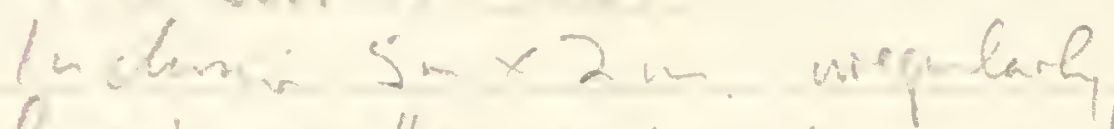

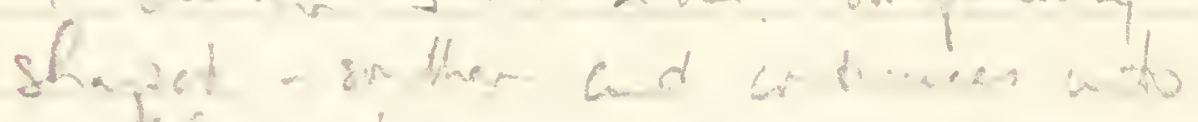
$\sin$ bef ond

bull inciald ded $1-2 \mathrm{~mm}$ She $\vec{\omega}$ 


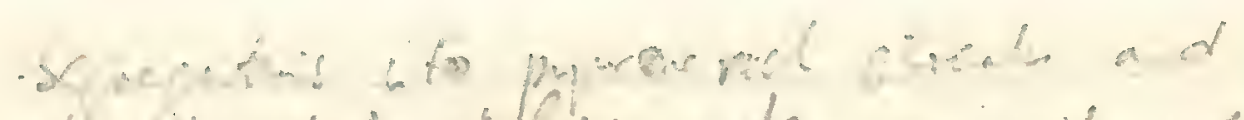

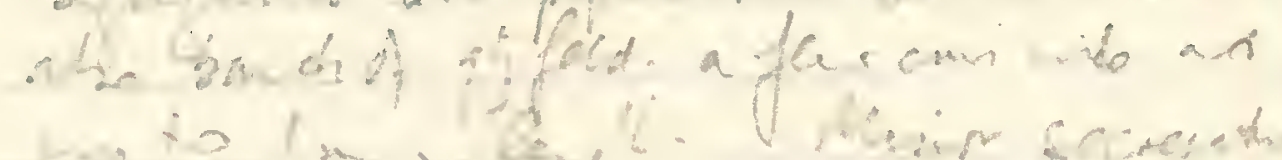
The ret.

Q temple 212 tale f intima $10 \mathrm{~cm}$

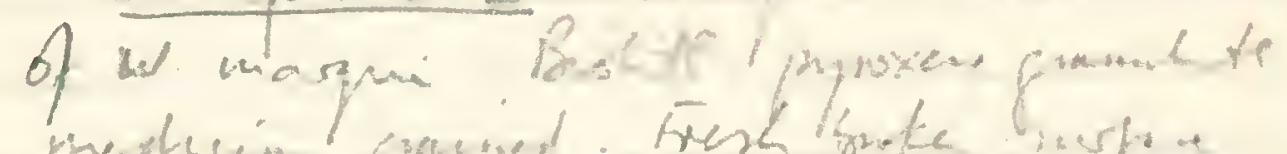

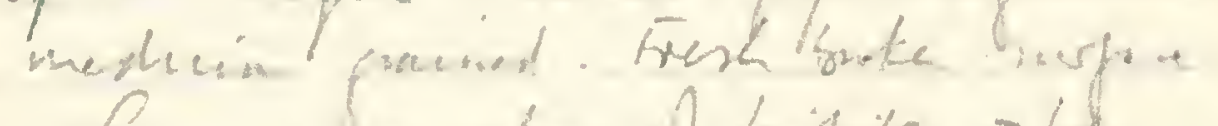

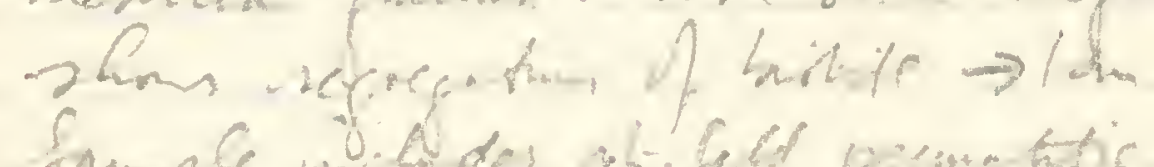

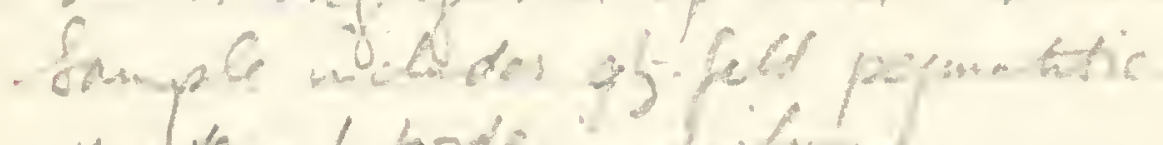

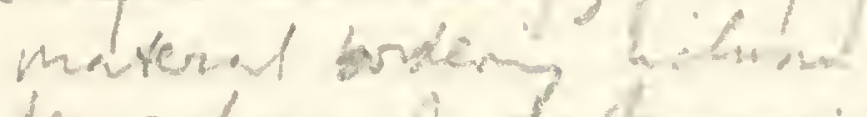
los achacel. dart muscid Sample dat whined to\% dak mus Leith due to the ind $70^{\circ}$.

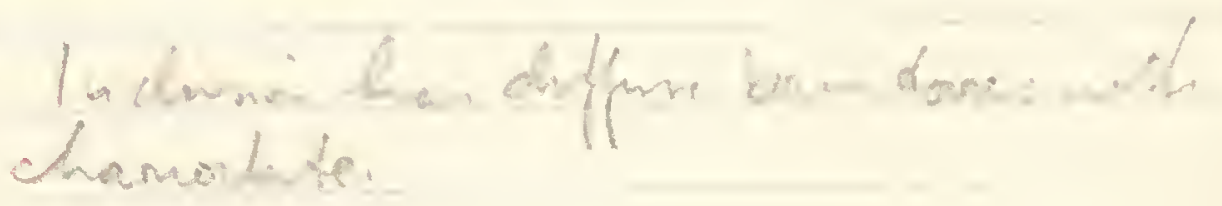


Kosterg Bland Manson SQ40-41/15

Qtimple $219 \quad 4788 / 5001$

Noit-cation past Af iscant.

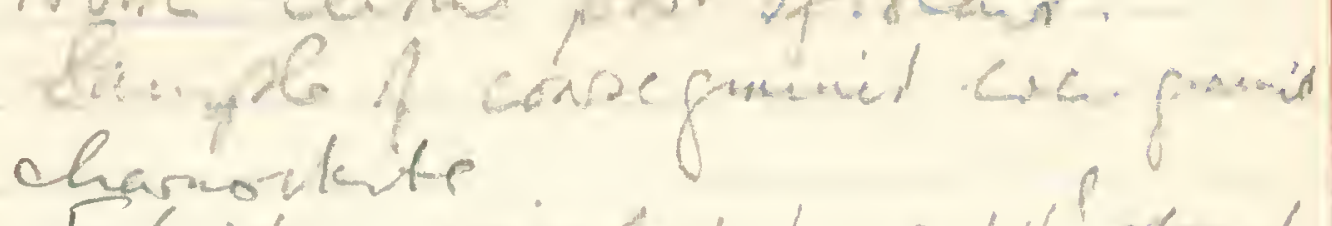

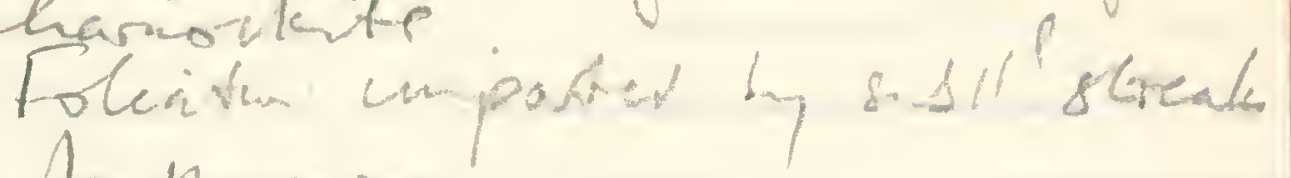
of pyosere

Suruple 220

10.

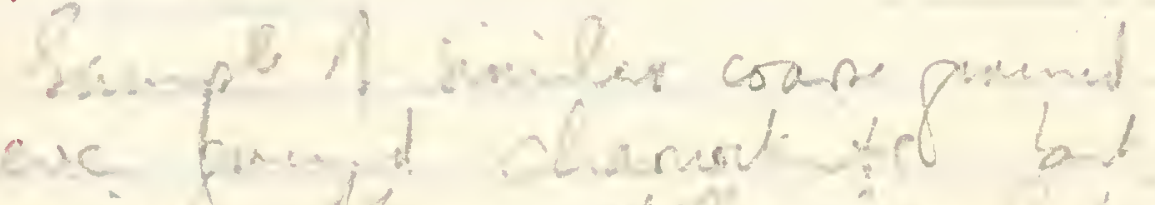

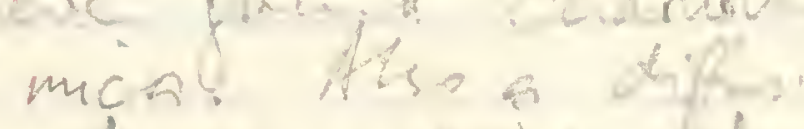

of percolate noth.

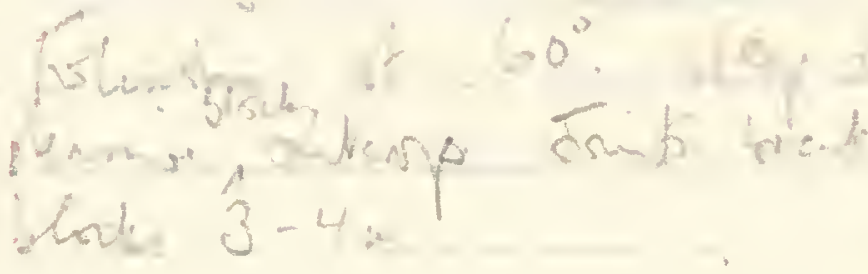


Gibbrey LSind Maums SQ40-41/15

ce. 22

$$
4712 / 5063
$$

foultcidplited new $\Delta$ sh Man rote th freinic eve finund coume point thanoktel Fhitew

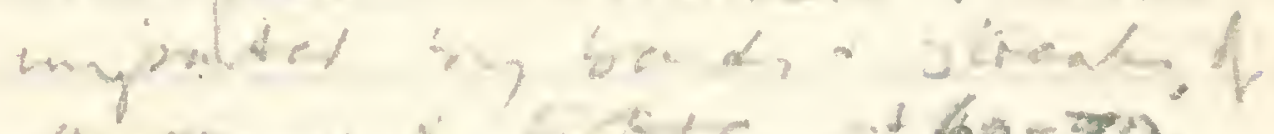

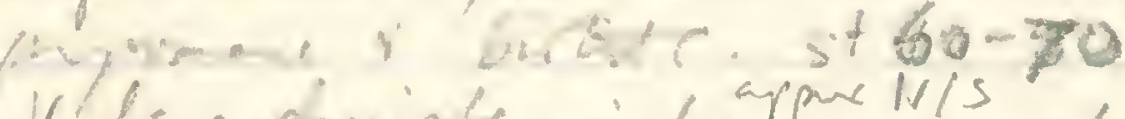

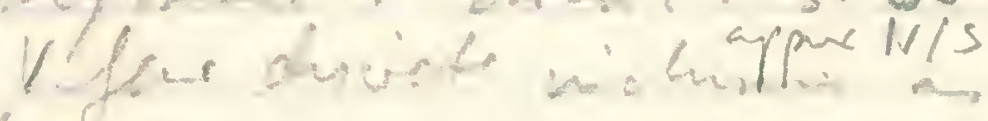
difing

Sompe 22:

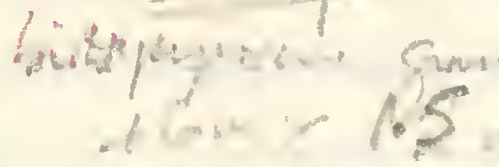
cat to \&f $55^{\circ} 75^{3}=$ Solpuy $11 / \mathrm{s}$. Sample 222 cors calli, $\cos 2$

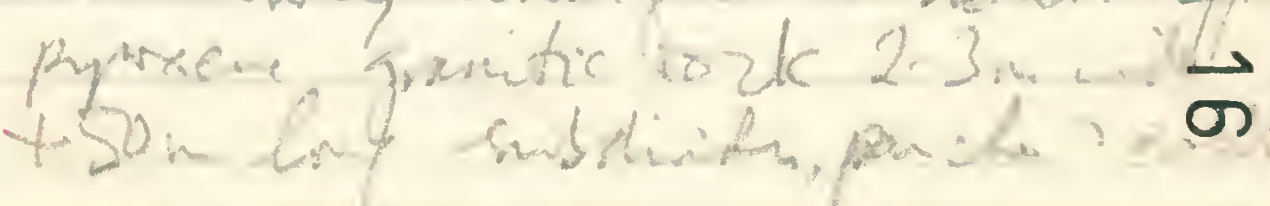




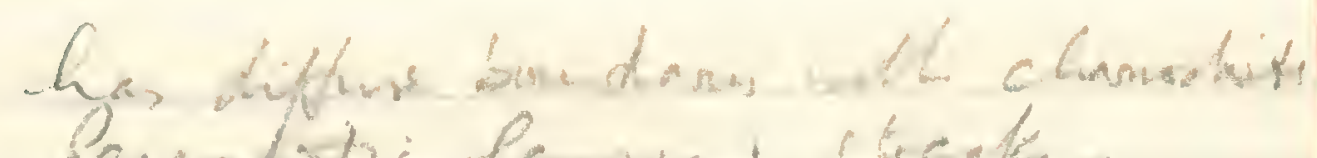
Pejertstic lewin's stente Somple take for nex maqu $\operatorname{lin} 60.5-6$

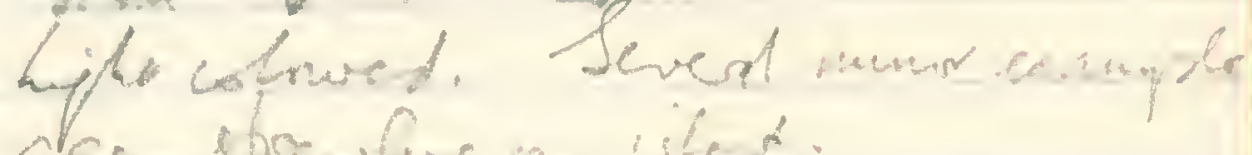
* 1.3 compar behmir

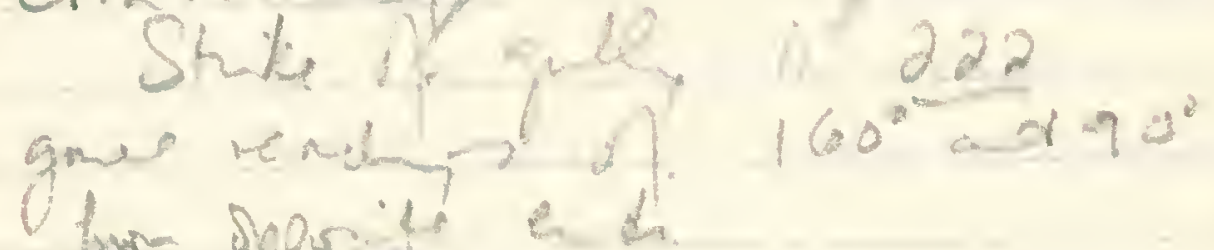
furmoposite en di

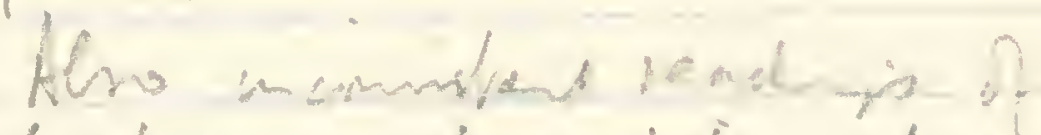
Whith in charmankte at beurel Conolutur ver 4 stm.

New $\Leftrightarrow$ sto

Sar pl2? I Connaprand eve

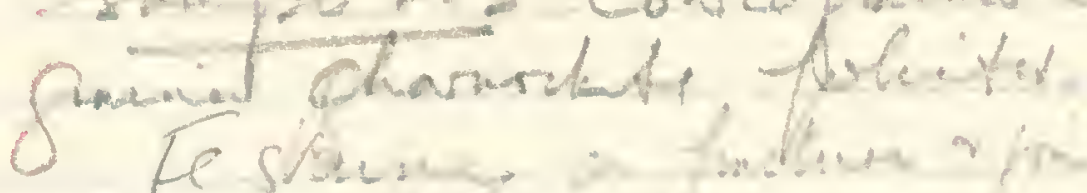

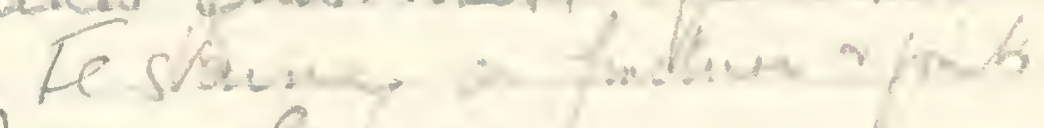
d) Smate ce.

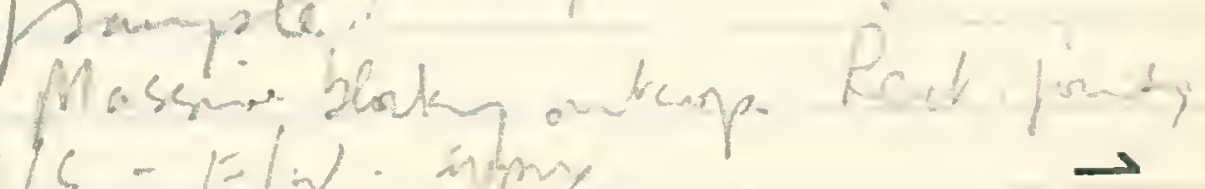

$N / S-E / n$ ings 
Simp $224 / 504 N 2$ Ast

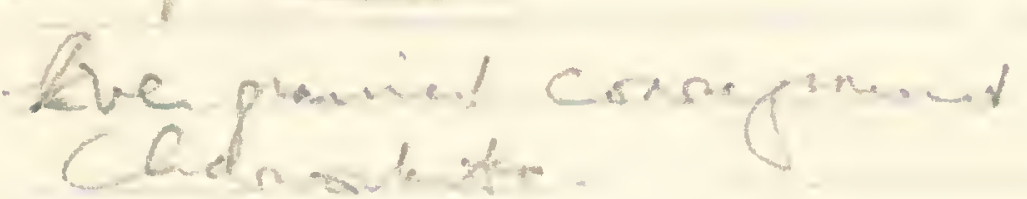

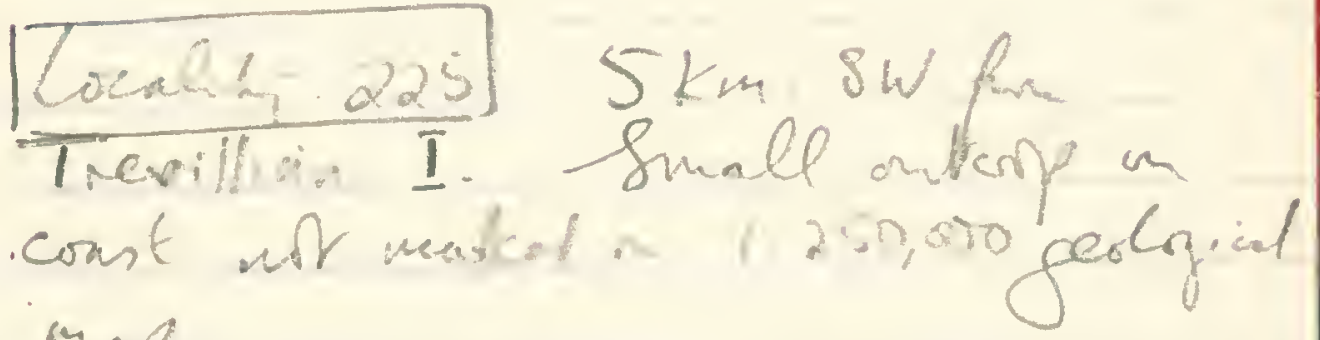
map.

Medrim-gumel chasnorkite with

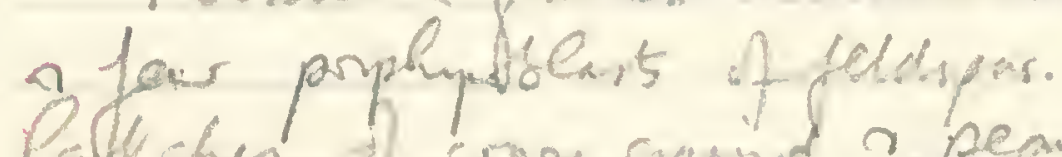

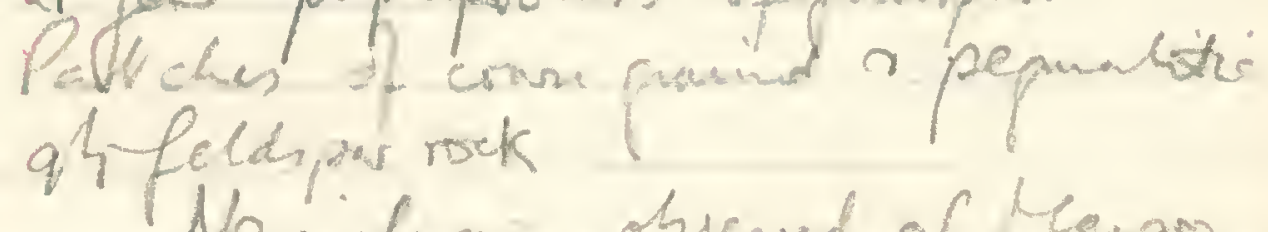

No ndermens obserred of tham.

Much moraine in bop of anksp varits of gram a gromets = morth

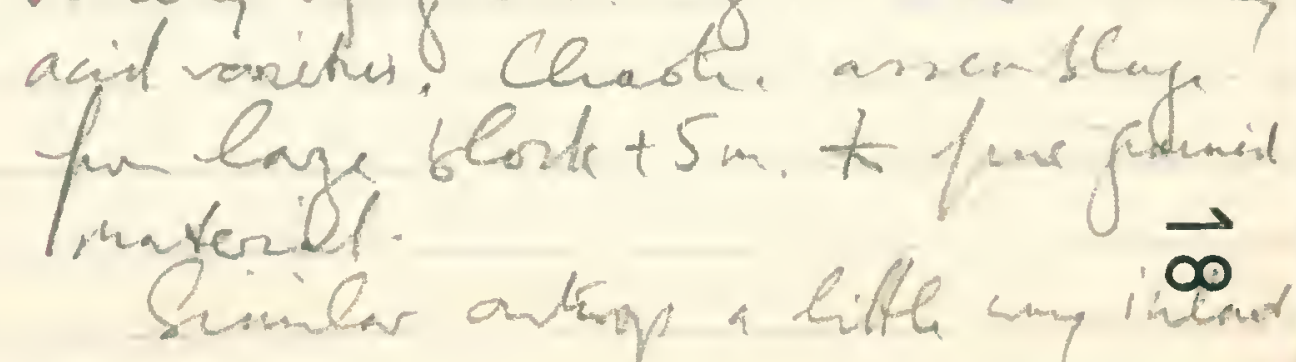


Manm 5Q40-41/15 226-230:4894/4695 Age det crllety M Rumex" fathen Marna Raye Landed w es 14 if it Bumet feak Lorality 206 100- Nupriefs. Circriac eve prominad chanocklete Coare quanict rock streaked ith pposcem in

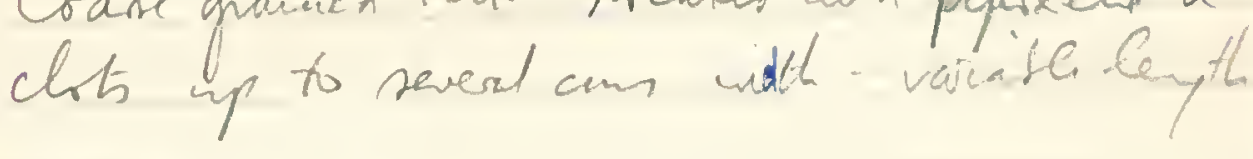
Sample 226 coare paned hium and

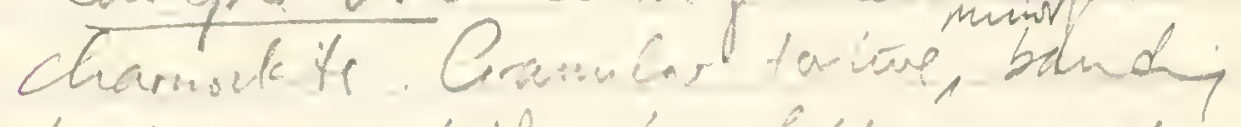

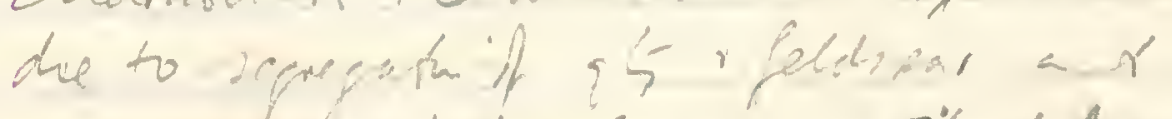

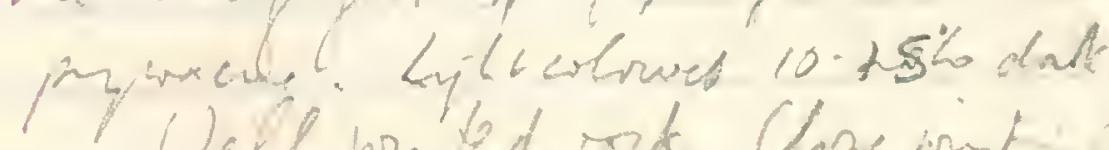
Weel pound rast Chrepint

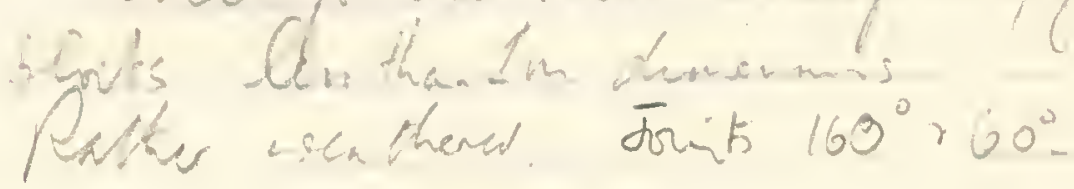
Sample 227 Neas 206! Inclunen A gamet bisiti fuem $1.5 \mathrm{~m} \times 0.5 \mathrm{~m}$ eler ated $60^{\circ}$. meduen monid and

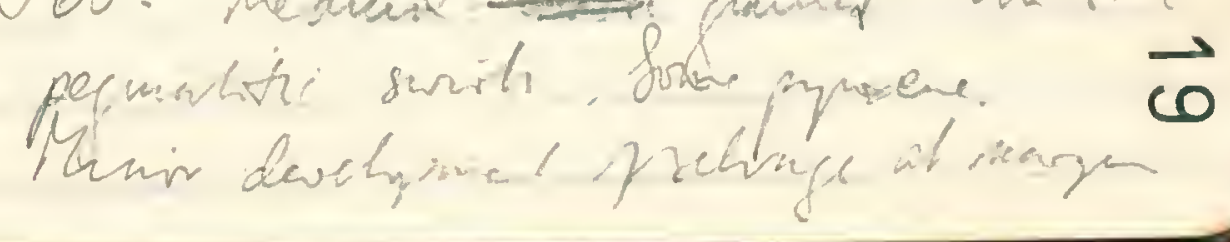




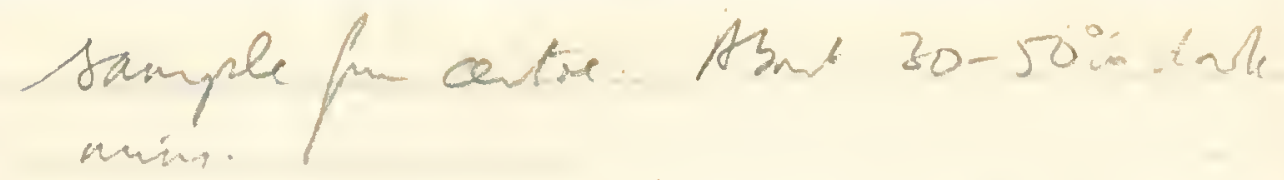

Upidge Jample 228. L

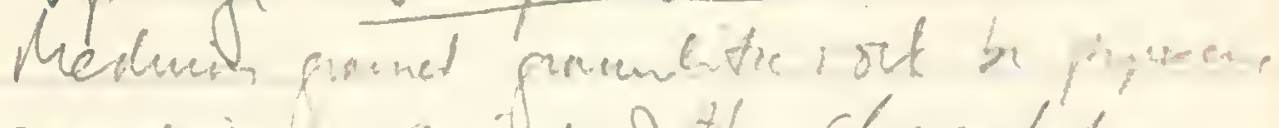
Gromerited vanate of the charonkt Munir band; ho peg. lewsin.

Uell minted. incal wito mu. ll blos bo

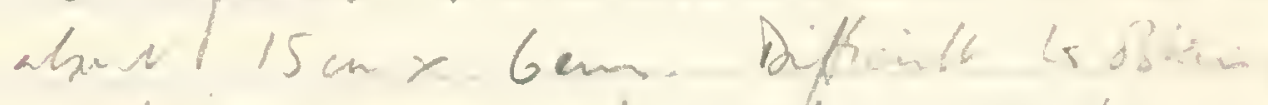

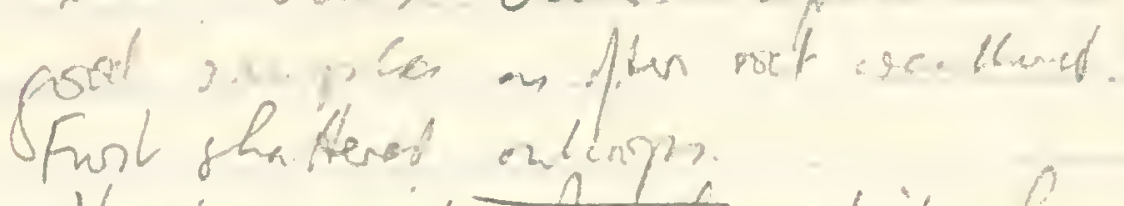

Varions varckin of ctarnokite for 226 to 228 wh negulos beguncte bands and fawre, Id's lameton e?

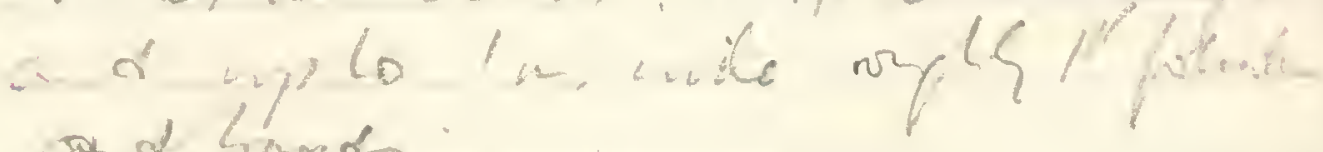
and binating

fow dewert?

A far minir thew zor a shot, $353^{\circ}$. Sample 224 Ar foith najenew cot thamsi cosos-gmined eve-pround Or mated 
Cample 230

Mare Bani sepregatuon "Whamortares une os pork 7 ryed.

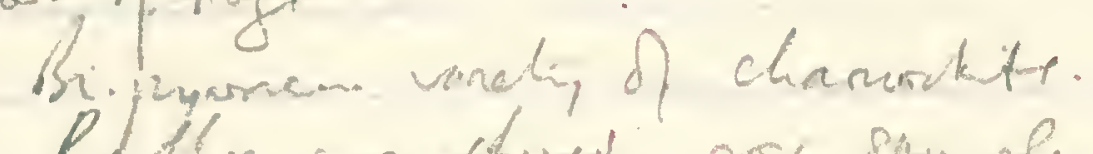
Qhither coe. theret posi staple.

flawe 231 $4895 / 4690$ Mamie colre pamiel cie guived charwakit fint a kers neas fost of ige math of col. 
FrIDMU $30^{\text {in }}$ JKN

Woschern Ntks Pawited Giness Mauson SQ40-41/15 4654/4806 VIt BLO alt readr $2710 \mathrm{fr}$

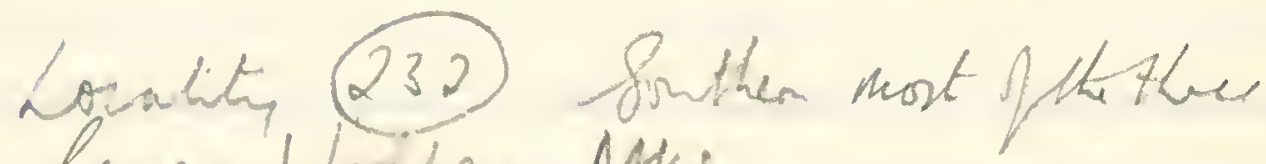
lager Wosibery NAKS.

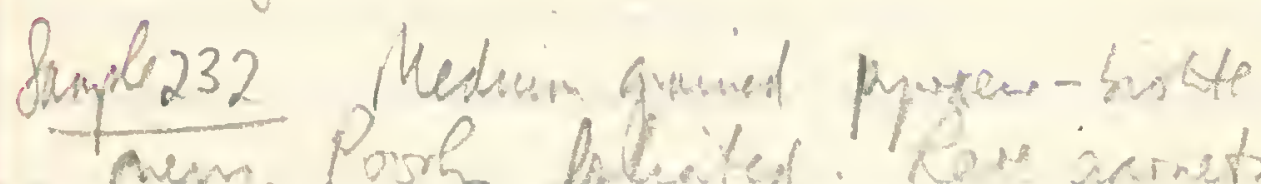

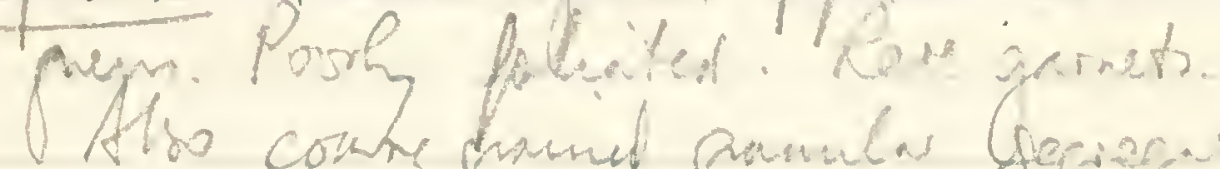
whe man rock.

Fol क5 60 ons 60.60

Well printed Font shatered Bloctes was. In.

Sumple 233 Couse grmid

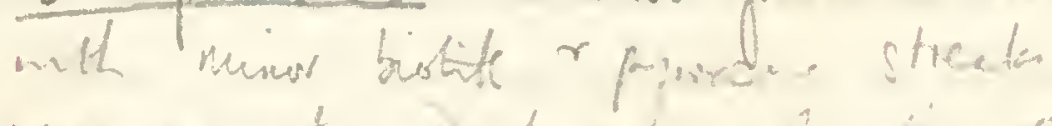
Bondles mben $15 \mathrm{~m} \times 2 \mathrm{~m}$. St. $70^{\circ}$ appor. 
Sample 234

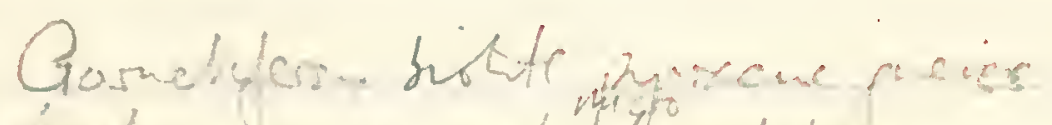

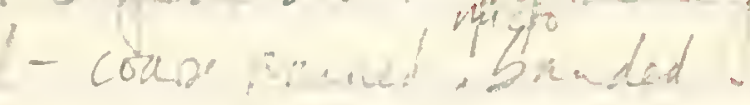

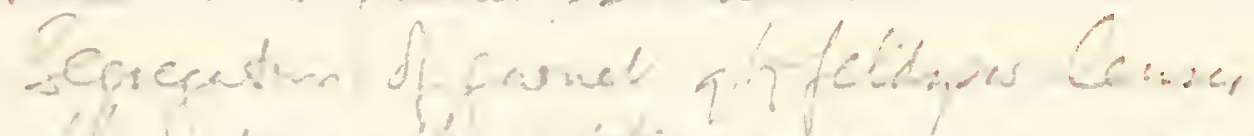

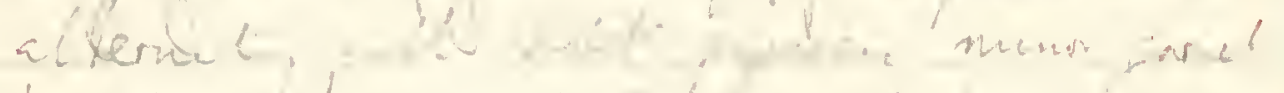

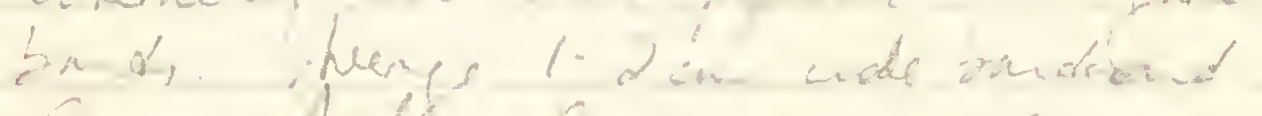

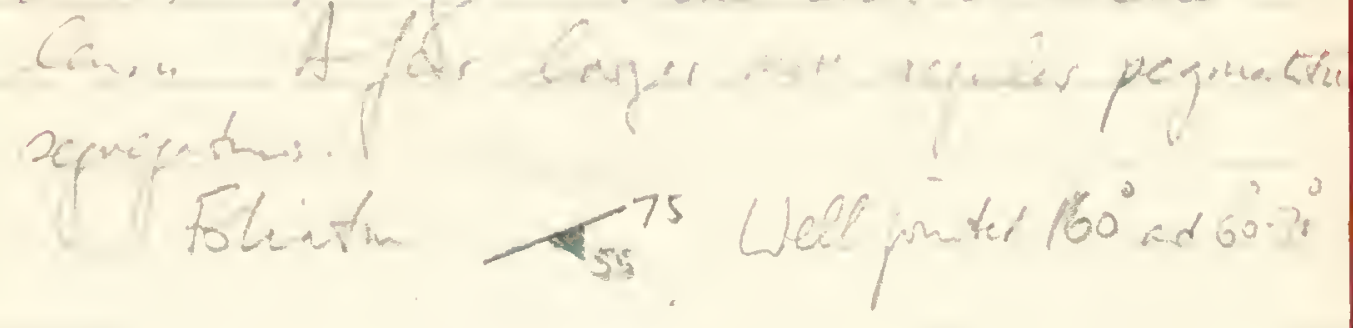

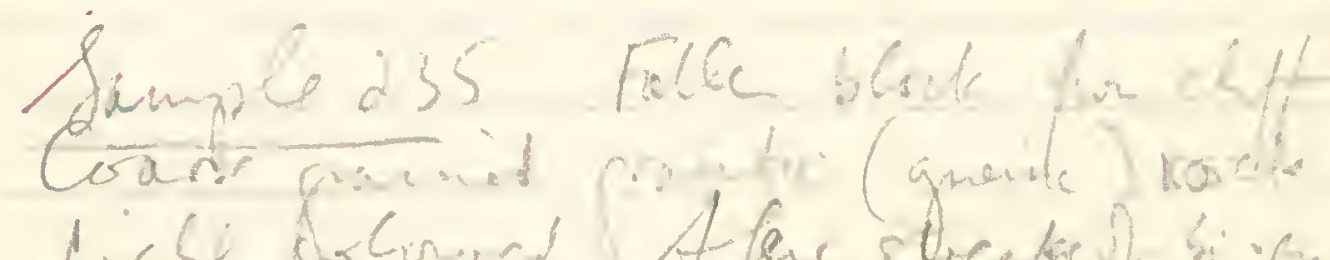

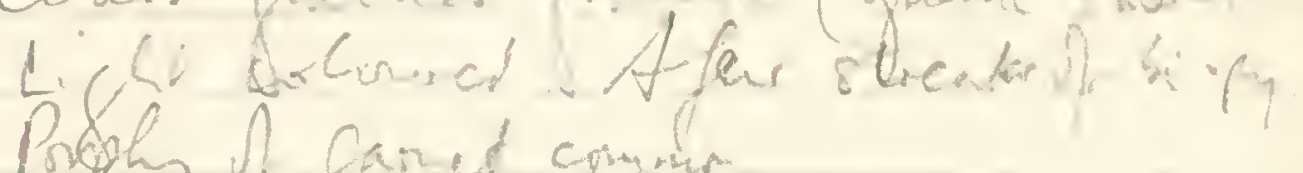
Popphas of ganet coming

Genend Gedgy

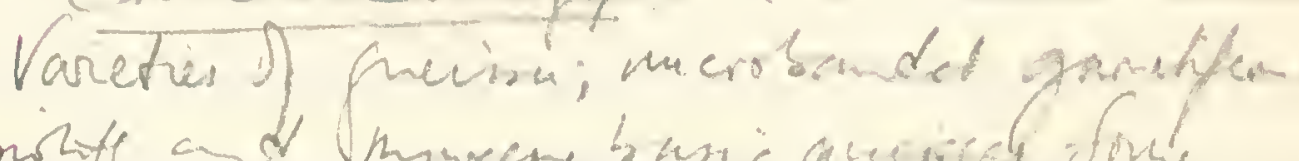

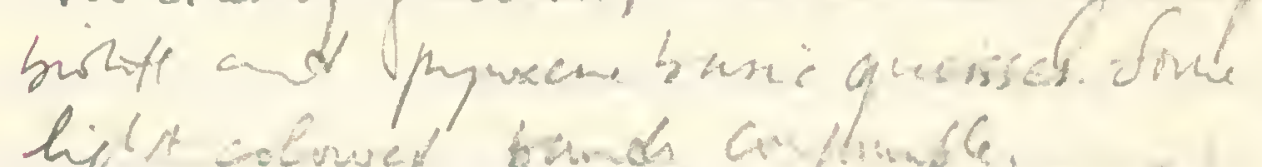

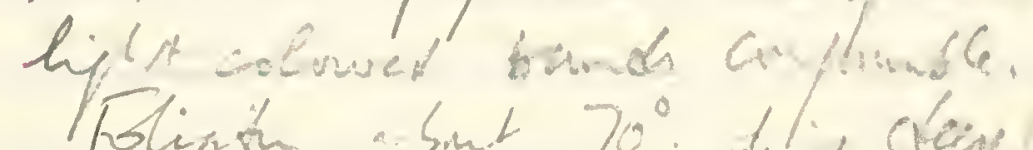

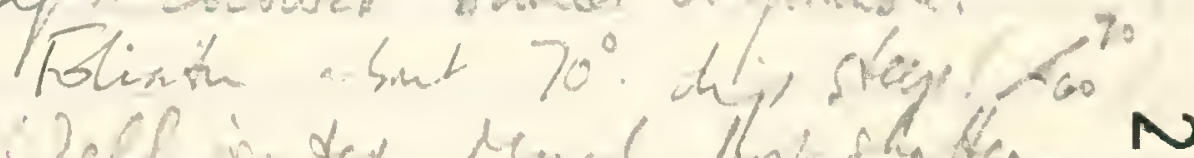

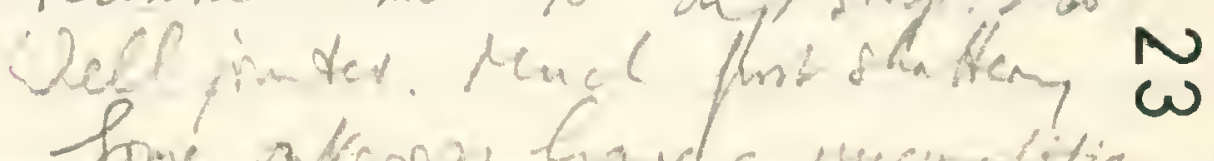

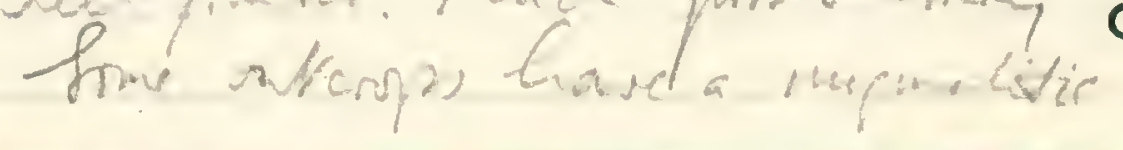


appearance - ireerear and deform bands

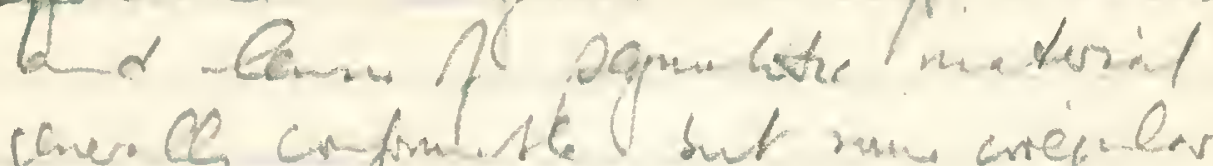

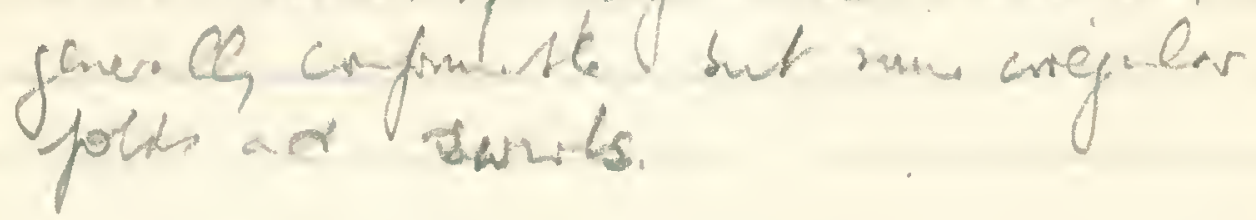


Sat 31: Tanum Mil00,000

A1. Rent Contrand SR 41-42/10

bugg to Rigé

Mt. Bensley $957 / 1966$

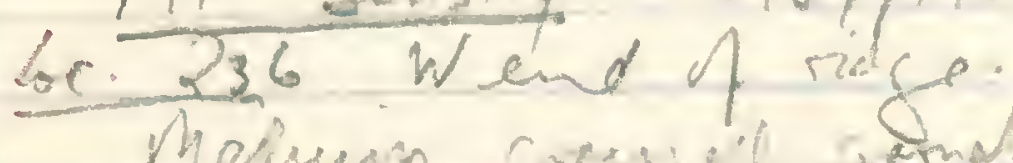

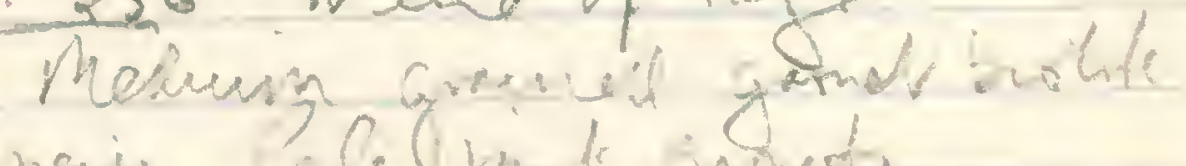

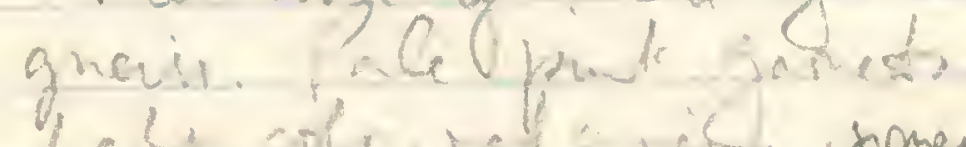

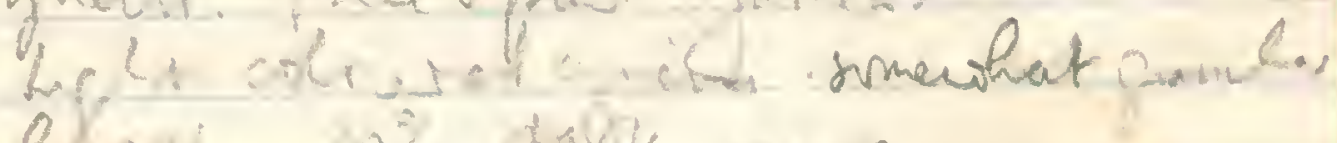
lentha $80 \%$ dabk muns

Heosh $1,1-2$ ms due $t$ dankers

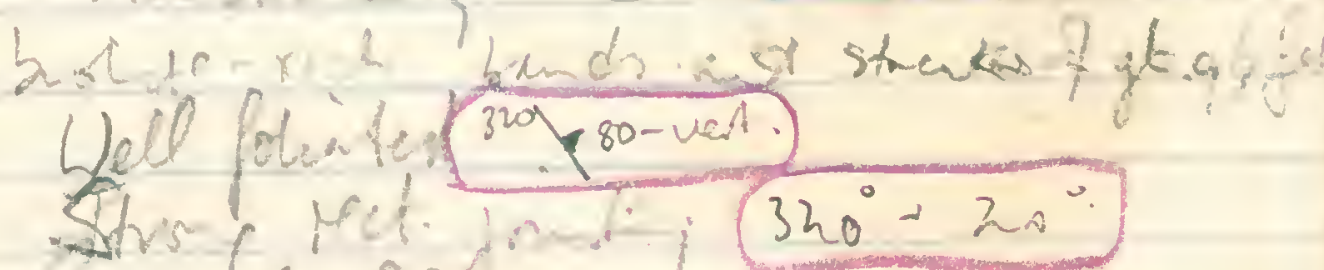
stricio $320^{\circ}+20^{\circ}$

Conlo

Brwithere folzames

Acole blenk.

$00 / \mathrm{k}$

fold irde.

ज 


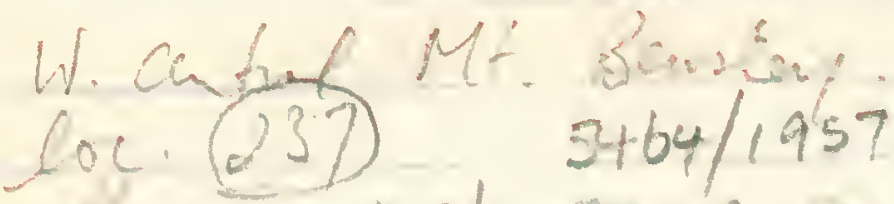

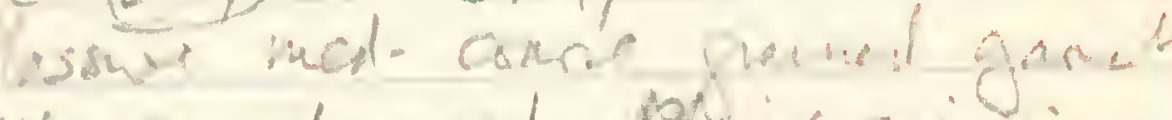

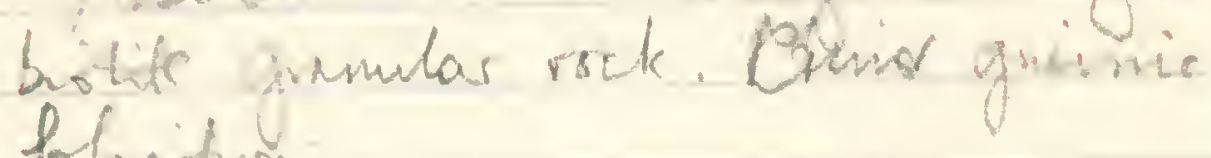
foritum

Massue ontery hot shetterel Lok

lase shats severil matres demain-

Crife alout $340^{\circ}$ vet.
Irom to $340^{\circ} \mathrm{N} 80^{\circ}$

Sinmple 237

$$
5476 / 1955
$$

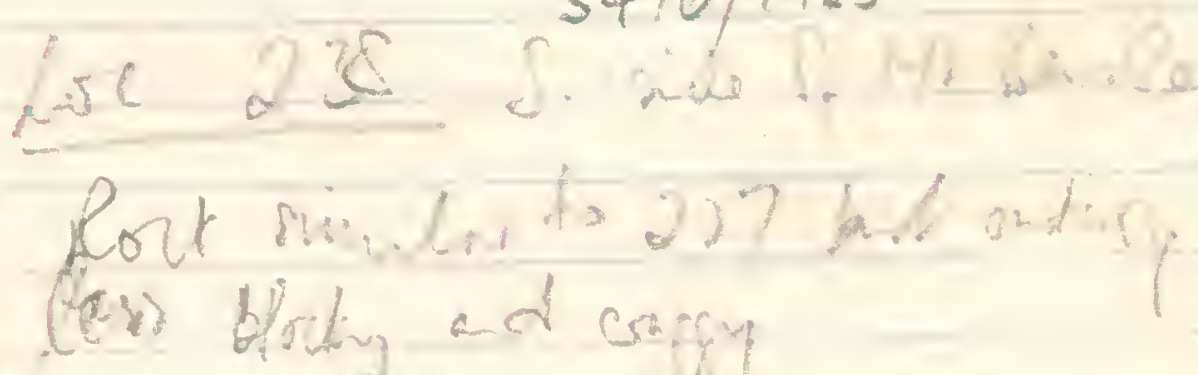
(ex) broting ad carfor

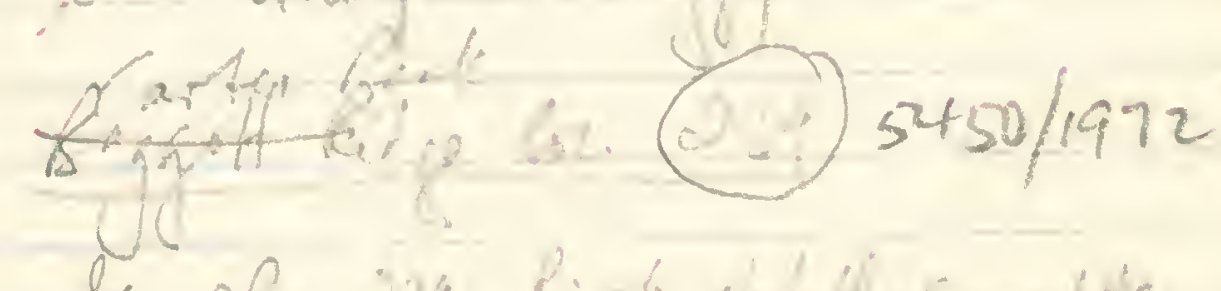

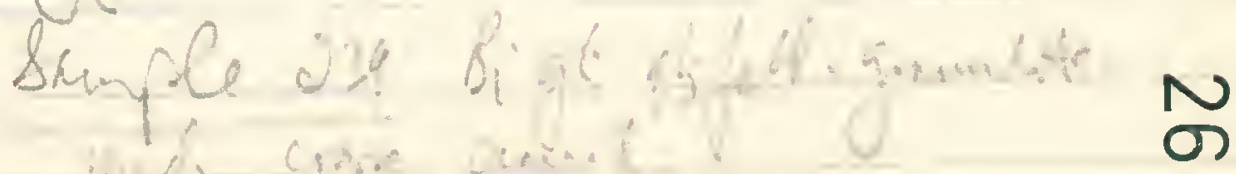


Sages Rige lo 240

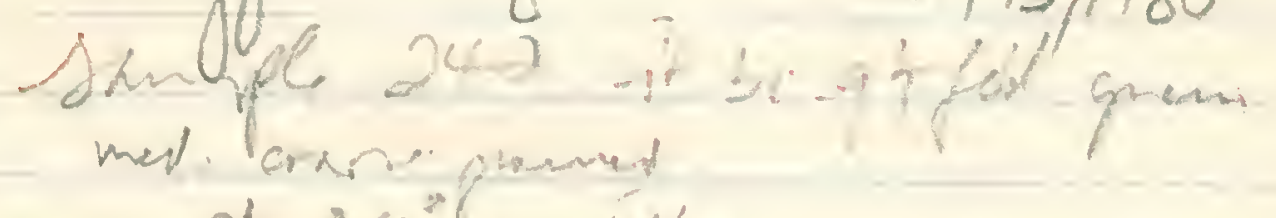

st. 34 ?

Varitien dut \& gt in

Bultam Nik Lse 24

$5523 / 1977$

Ahe gha ita -40

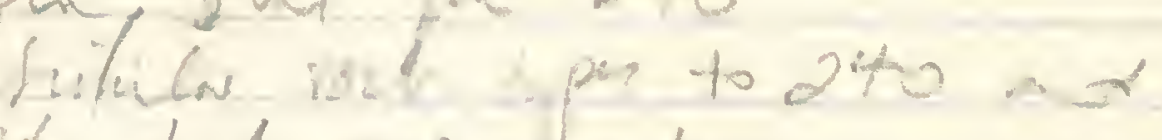
236 knt sonot visuasing

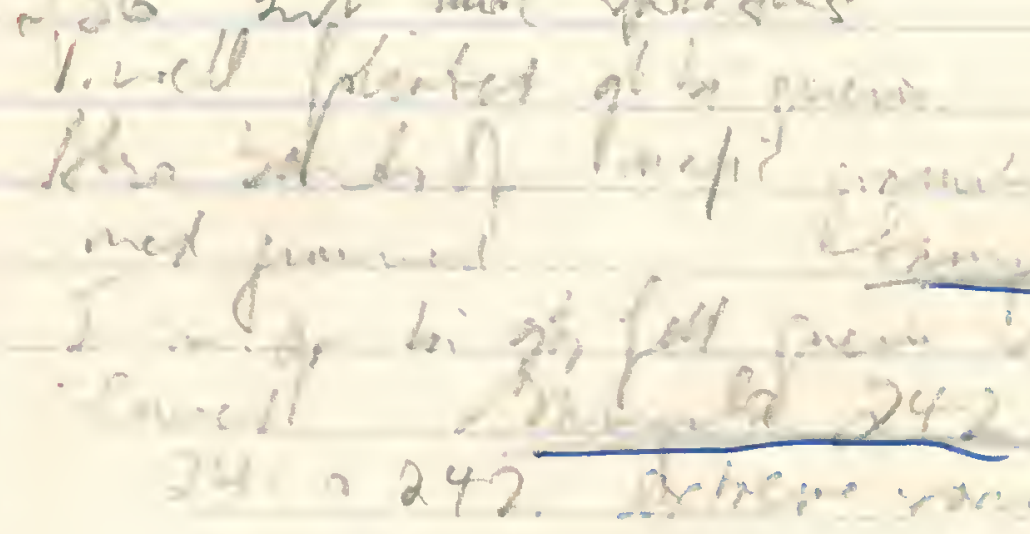




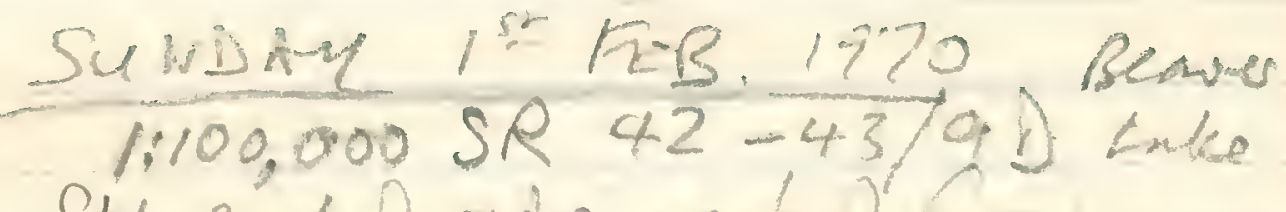

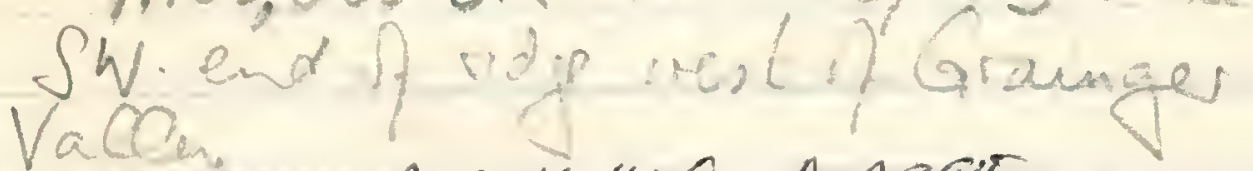
Vace MAN'NING MASSIE

$\operatorname{Loc}(243), 4480 / 1438$

cleff - gamet

the chiff

boikg foliter

rint

Shamp bow dores

(i. $240^{\circ}$

No 


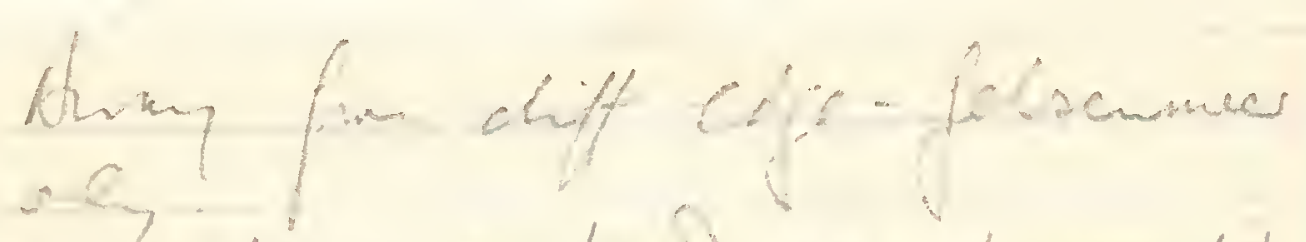
sampl 246 anchen ganel prombs

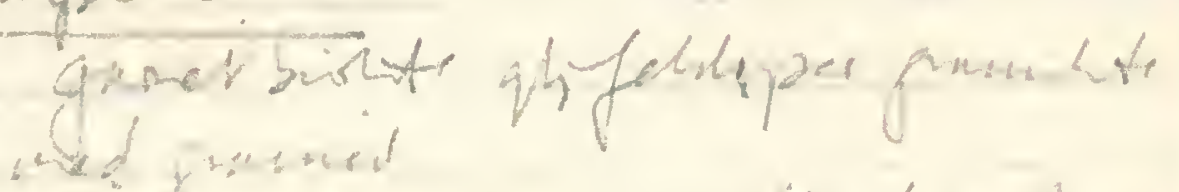
Aylus colnued a weithend mergnes ha foes busfom dase be tarle

variun of $2+3$

Loc. 247$)$

$4462 / 1462$

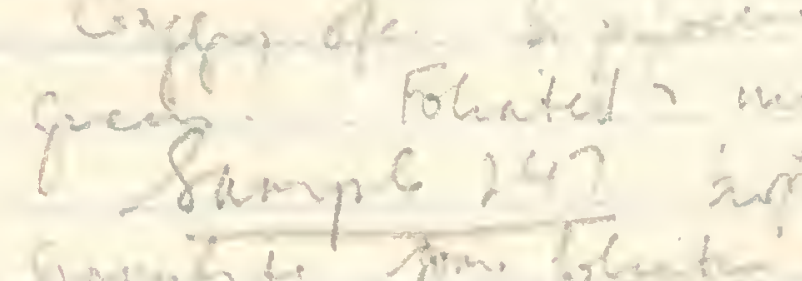

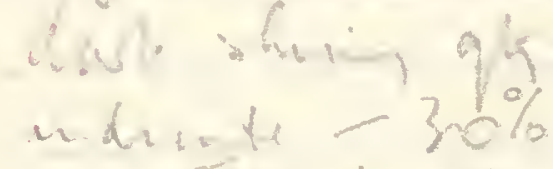

Fhath 


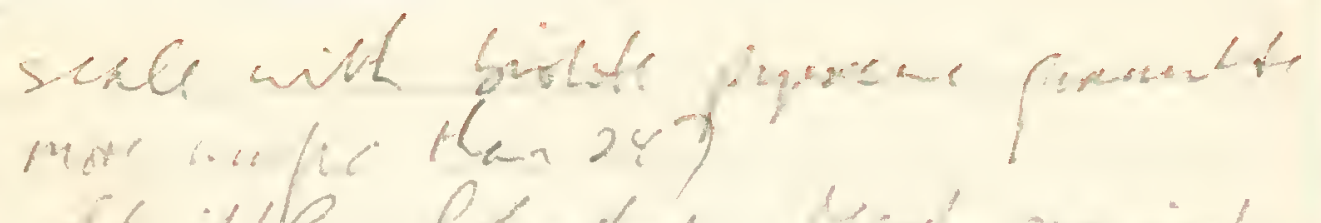

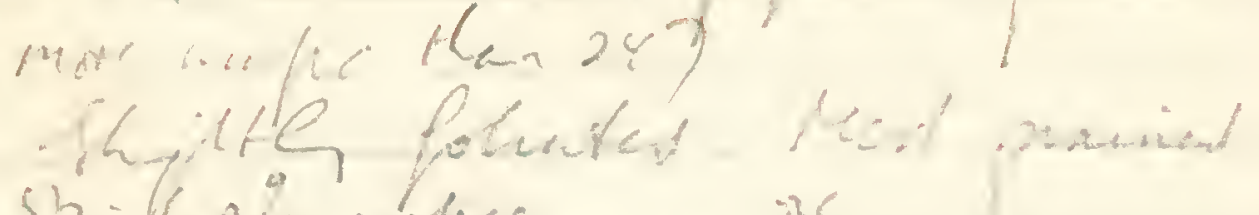
$56-1602$ mates.

Sompe 248 fol $x$

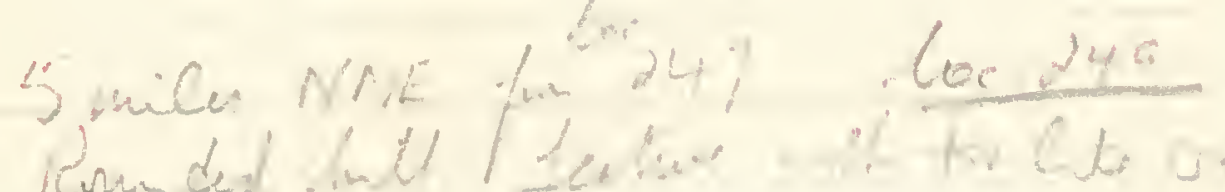

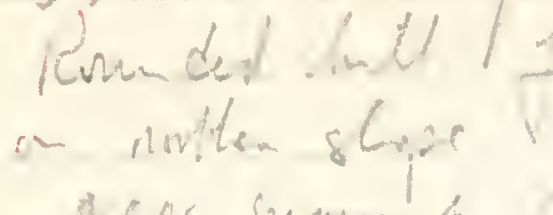

Neas sumoming

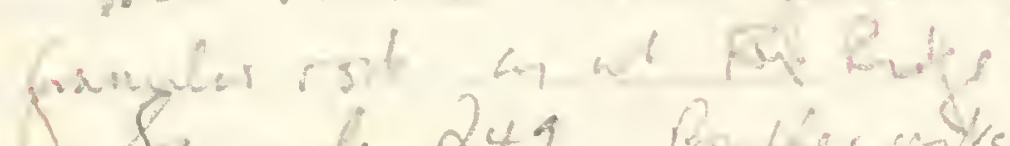

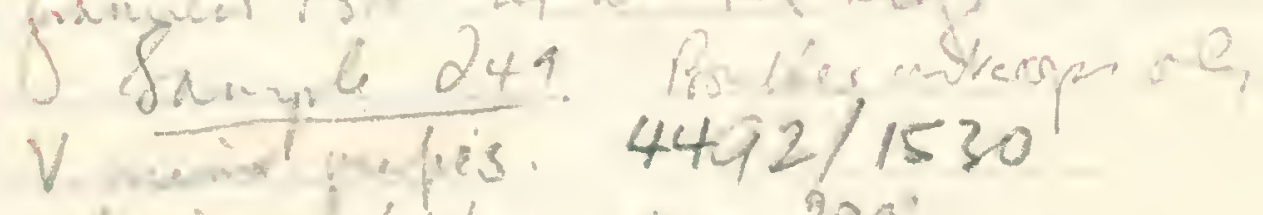

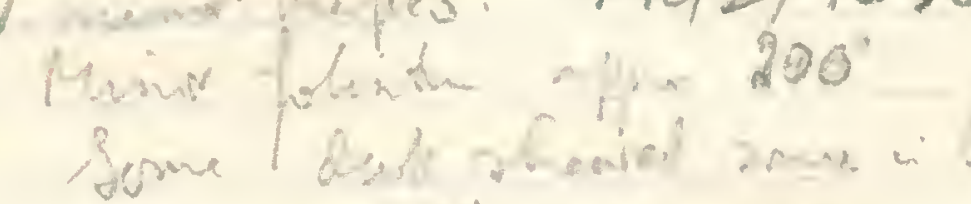

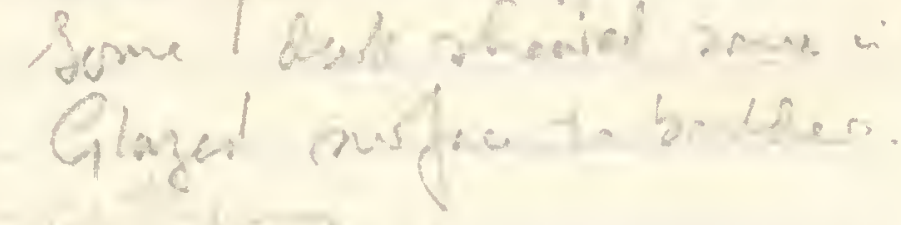

the fort

gromelits micilat to 24

omatir.

Foxprye ane

$\omega$ 
$t_{p}$

$$
4546 / 1597
$$

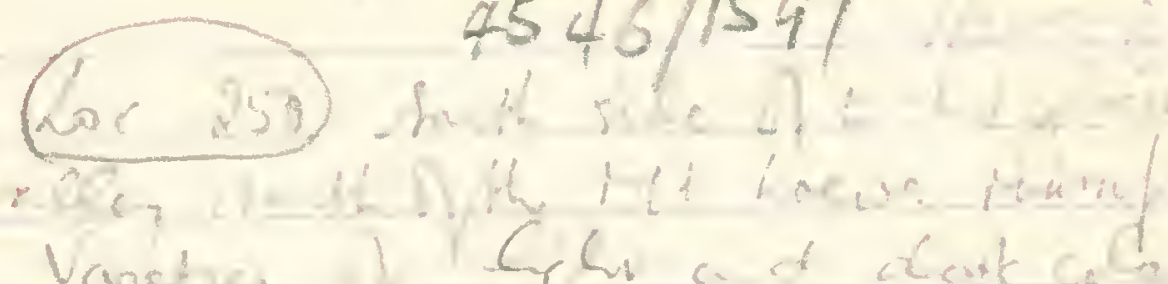
Varatue 4 Geh

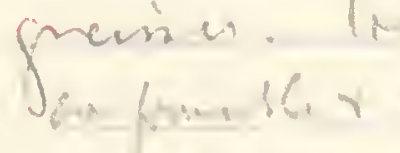

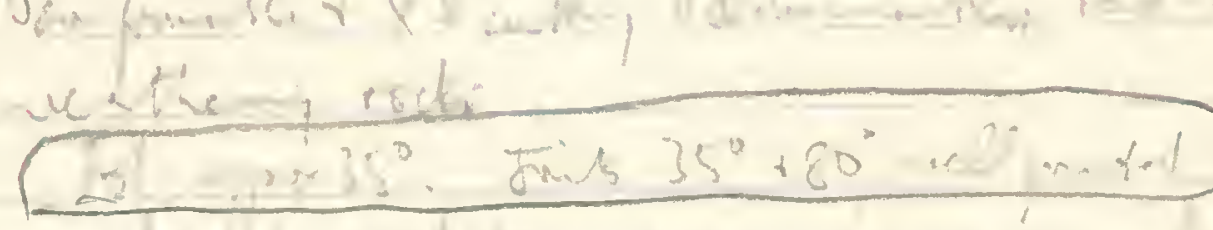

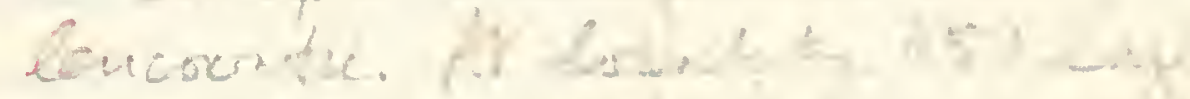

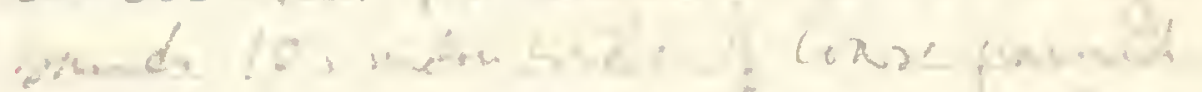

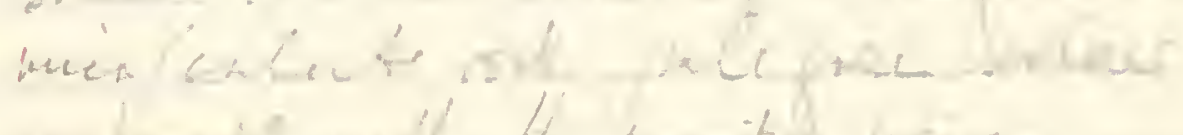

$\omega$ 
$i c ?$

$\angle a 1 \ldots$

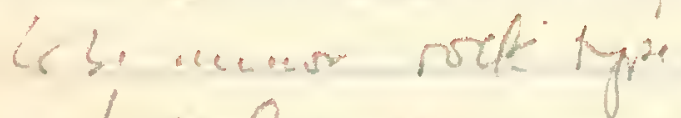

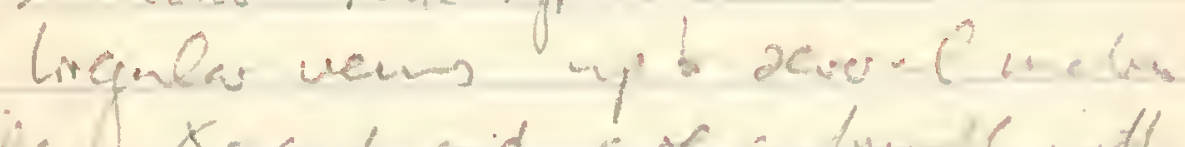

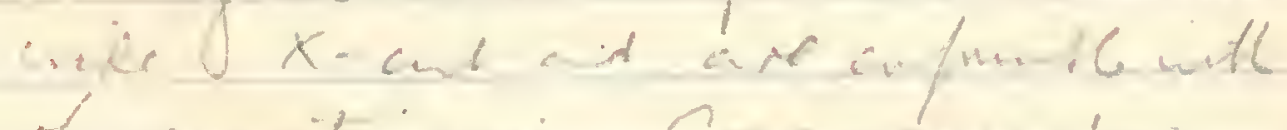

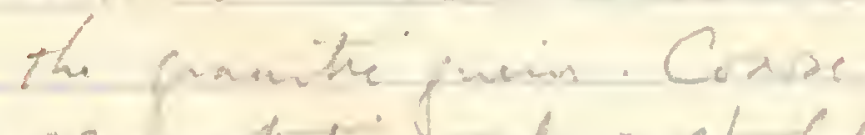

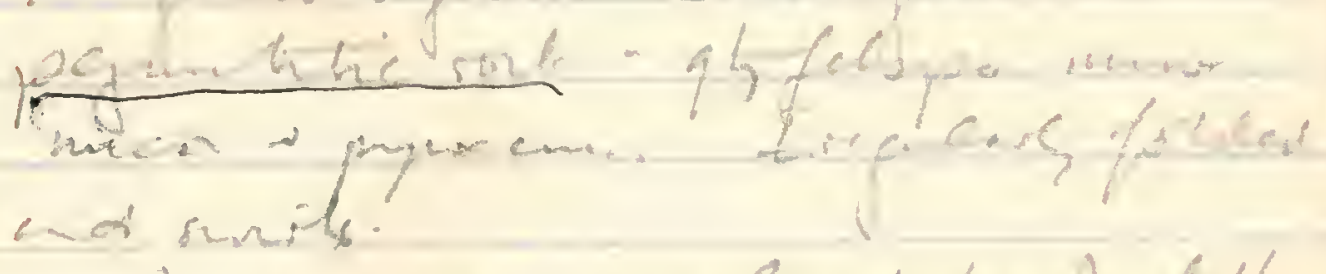

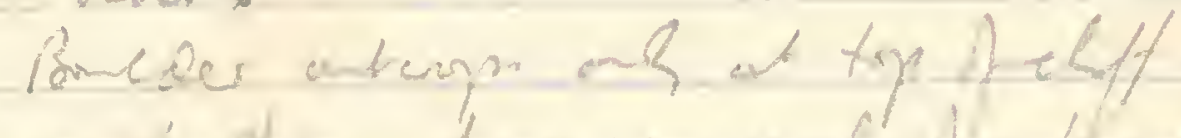

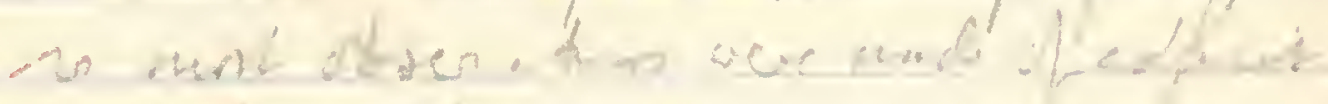

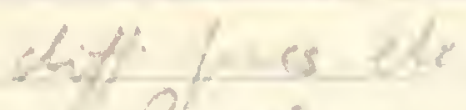

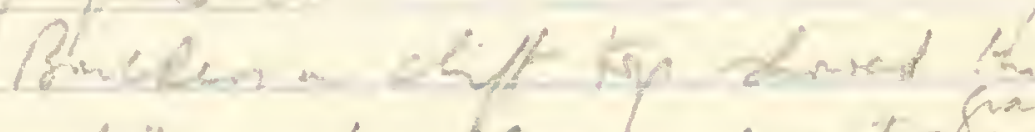

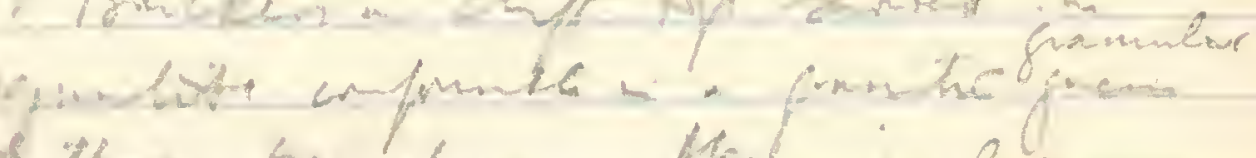

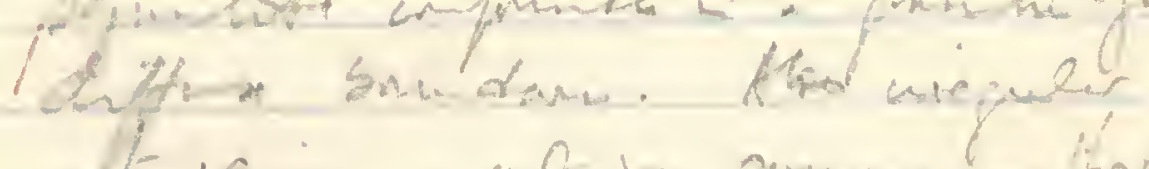

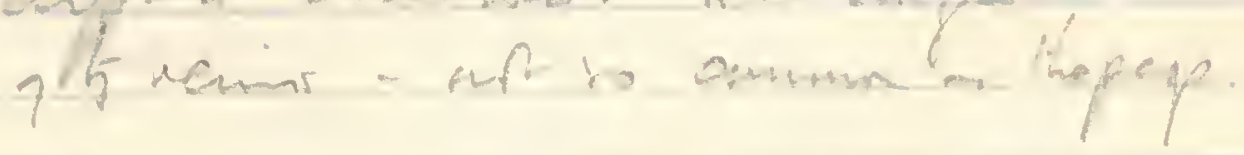

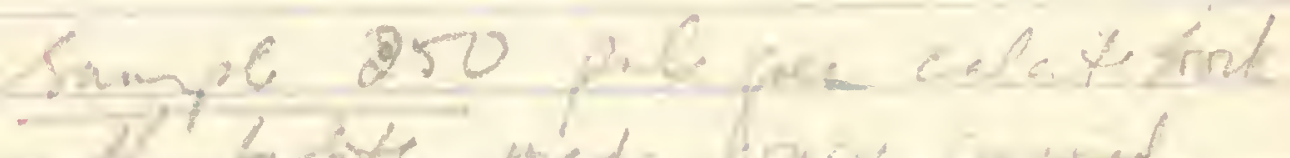

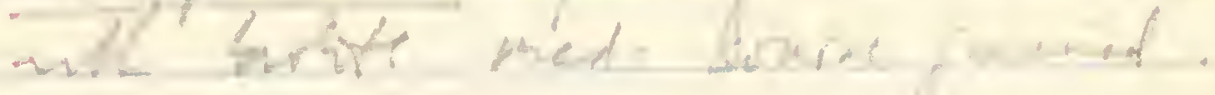

Sum 25

Peproming

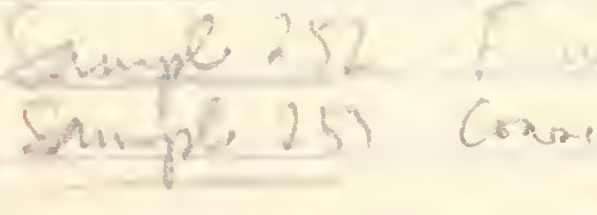

$\hat{2}$ 
punte. 4and bist

$(254)$

$$
4504 / 1670
$$

simpe

Phigh back to Moore Promsust

Sandiloud Nth - charakx

Nortside o wet Ma

ME Conperavee bandel sem 10/10 hile. det.

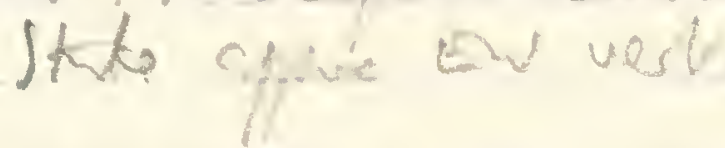

Gurther wate simber mot.

fot hip ber $70^{\circ} \mathrm{s}$

$\stackrel{\omega}{\omega}$ 
- Thimpirar Masiff mall cile

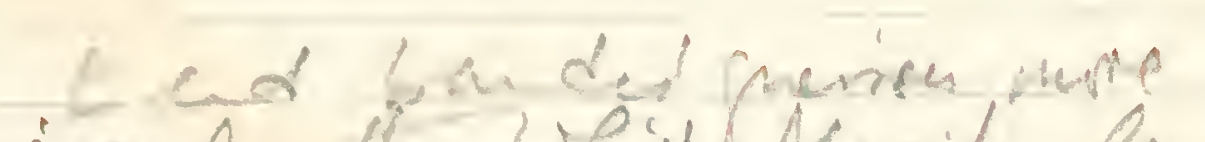

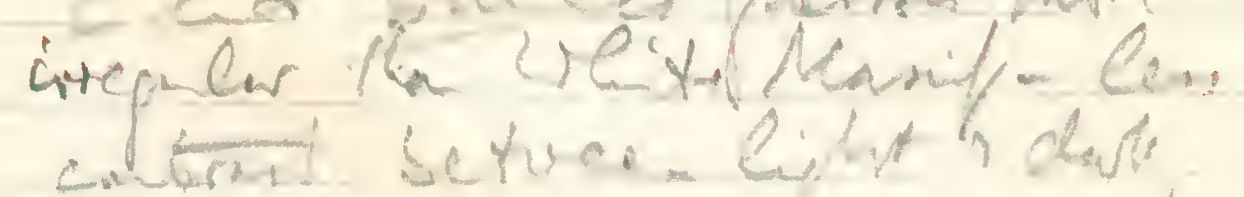

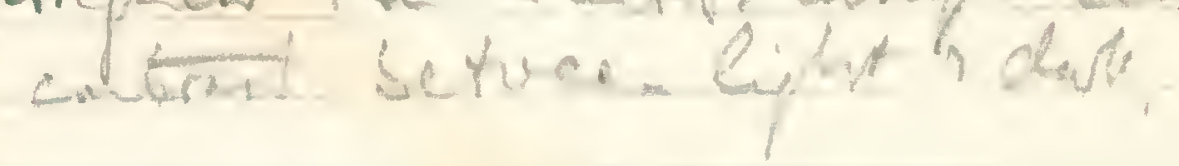

4J. and ontho ret-bim gantic an

Mr. Mbss Red. bmo frem

Mt totu Lecke Pothr Rues flhed bandel perinos

Mt Gardner wipulabe thidet gnersion (miprithe?)
$\omega$
$\omega$ 
$1: 100,000(R O H)$

MAssif SR 4-42/10B

Heso Shoto instiro R.35

MF Jedel, - Mtsharlit $8201 \mathrm{~V}$

$6054 / 2004$

sole feram las

whe ganes

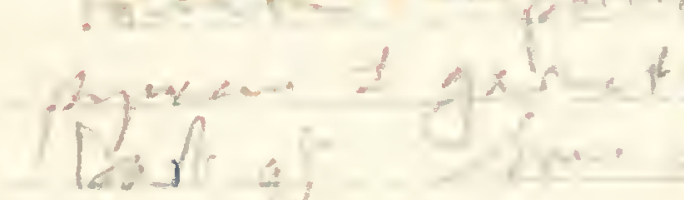

Fis?

$\mathrm{HeCl}$

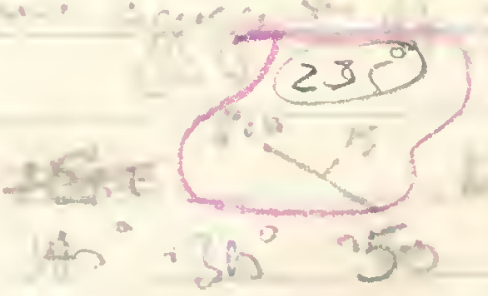

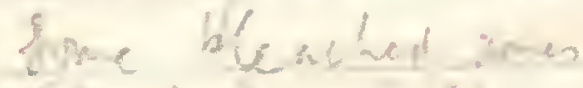

$\frac{5}{3}$

loc 256

$6058 / 2003$

$\stackrel{\omega}{w}$ 
H Rue to storat

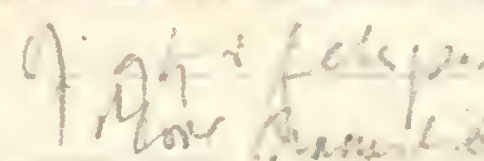

ganels

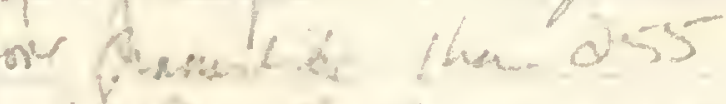
fol phomat

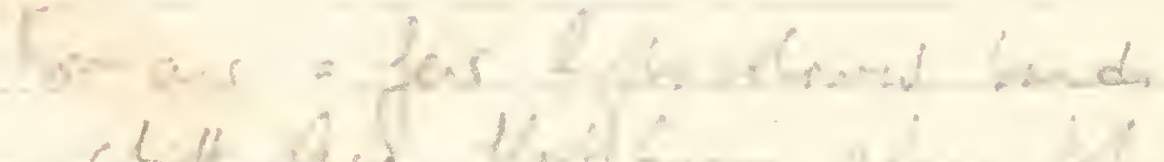
2 chy that fority

1-15. Si: Ite Ale samples $5987 / 2142$

$\operatorname{loc} 257)$

Af 4 Sin

(Cos 60 Bix. FI: Shalker.

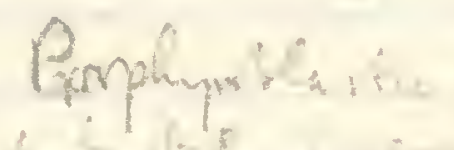
neconis det $\omega$ 


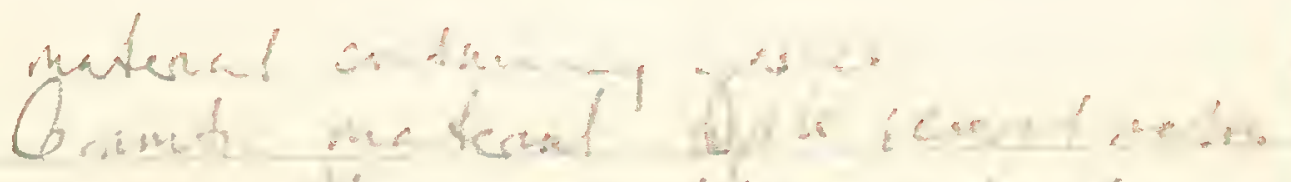

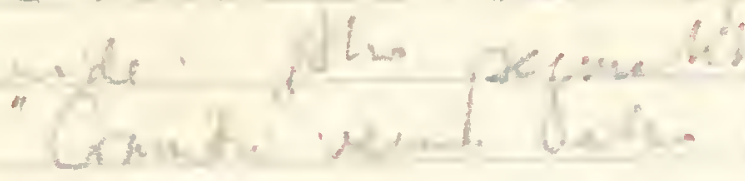
truenson

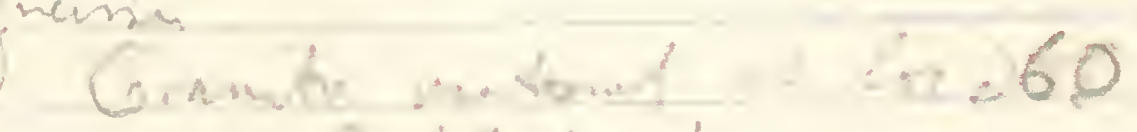
$-i+30 \%$ of ktad rsok

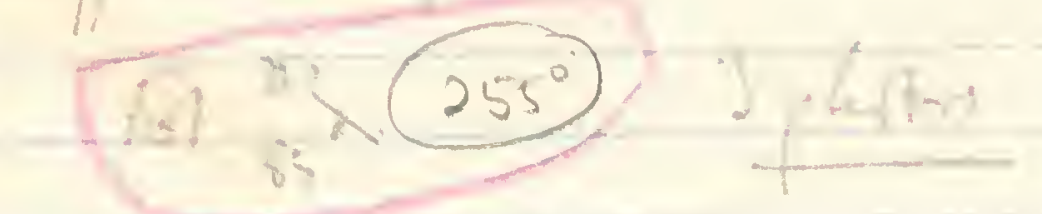

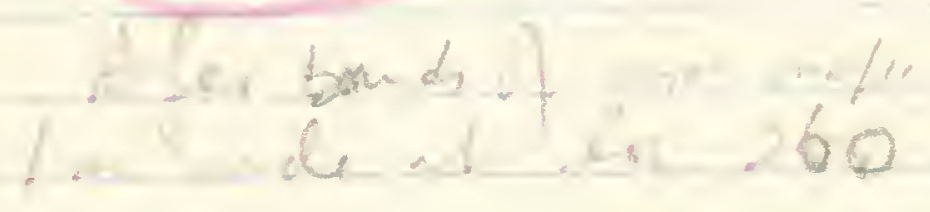

Elsushere alon 4

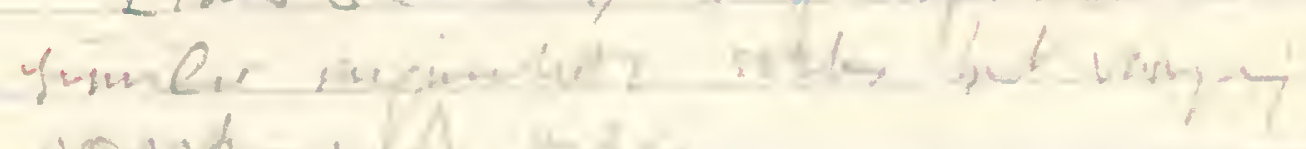

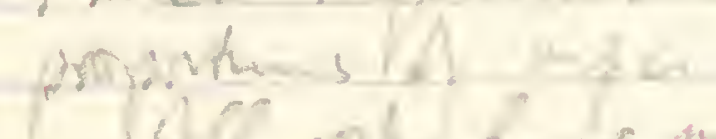

$\left(\frac{1}{6257}\right) \ln 25+7$

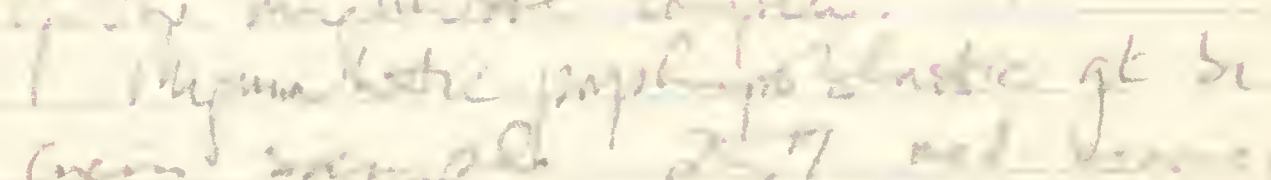

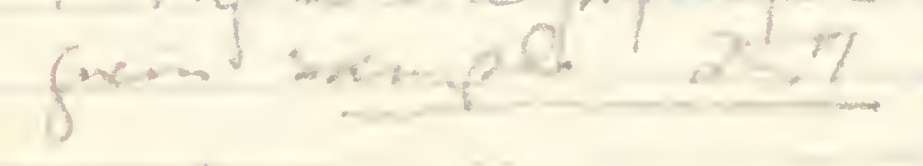

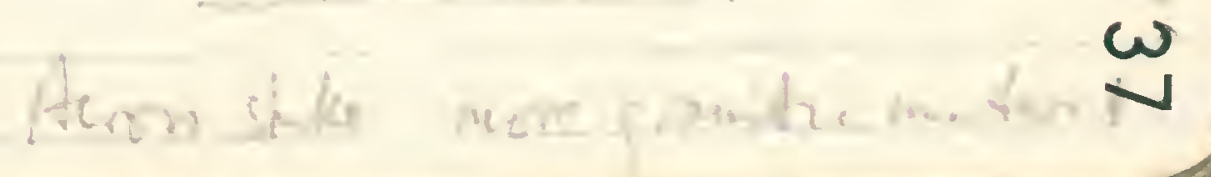




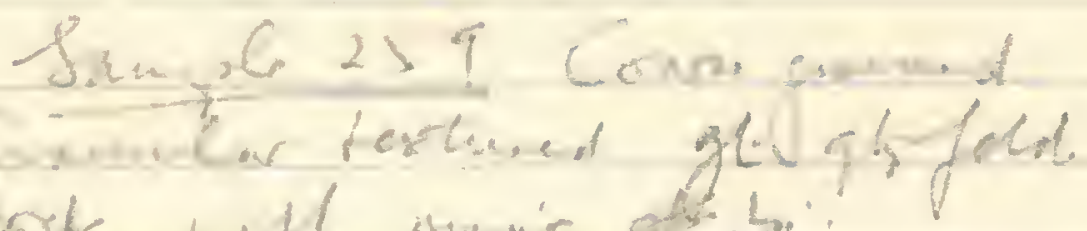
Fote with munis of bi

Fre nir

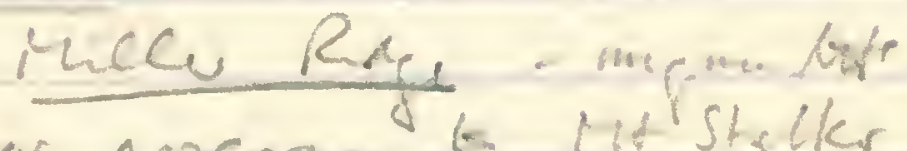
nimber appean o to ristelks theol indated ofe in theil

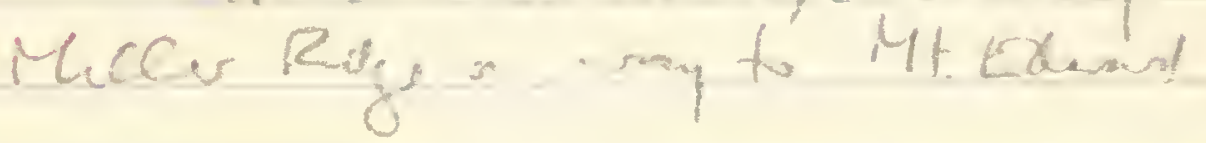
Sumple 262:205 Mt. Edward 5947/2067

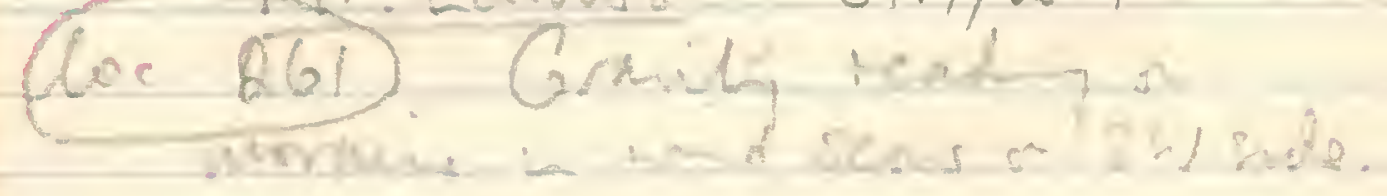

$(\cos 262) 00$ of if ited

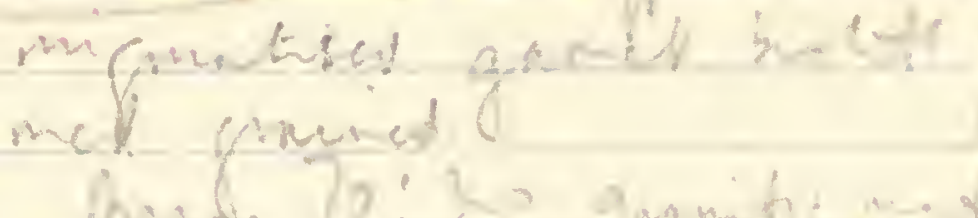

$\lim _{4}$

$\omega$ 
$1, \ldots+\ldots+\infty$

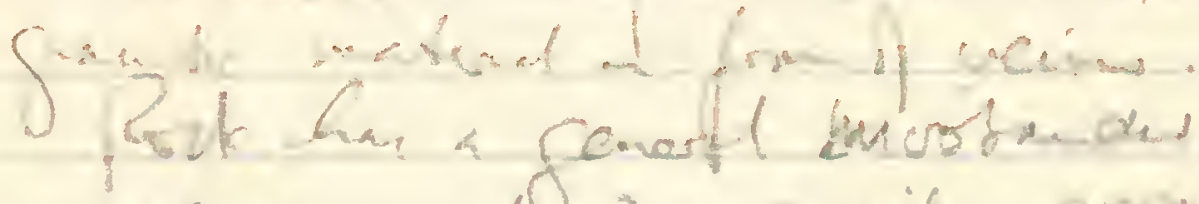

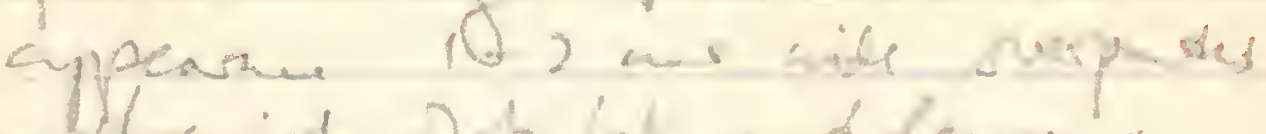
Wh kirh of $x / 4$ - A kems.

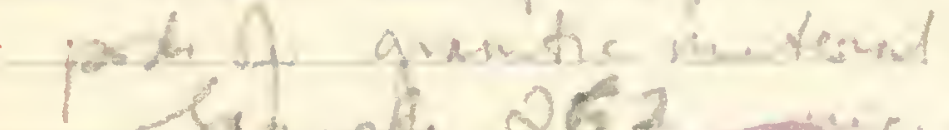

Somple 262 (285) gl busto fhein. Genent folied 20 bow t $-4 \cdot 5=5$

\section{$8 \pi_{n+1} 626$}

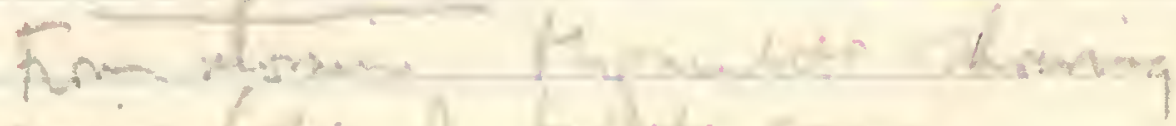

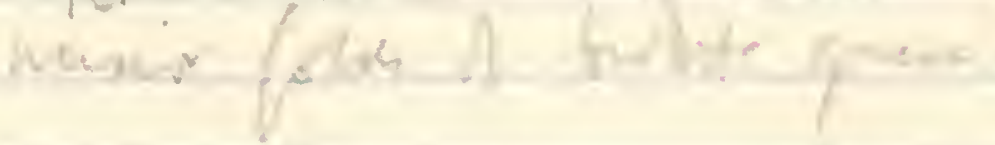

\section{3 phins}

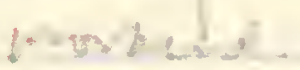

smill seale pryometic poso

a. Jen 
famples $265 \times 266$

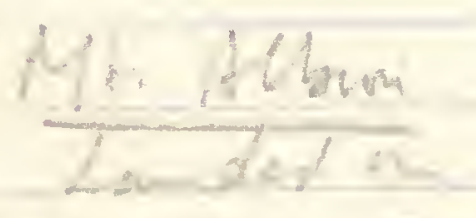

$5997 / 2004$

folickl. 3900

1690 he

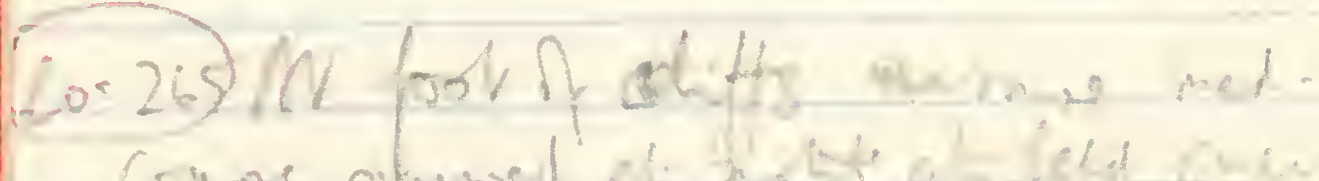

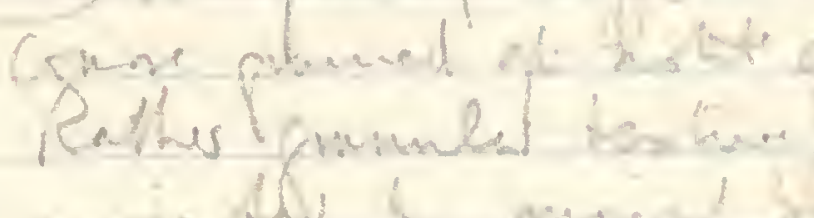

$\rightarrow \lim _{1} \operatorname{lo}$

ind

arod i-?

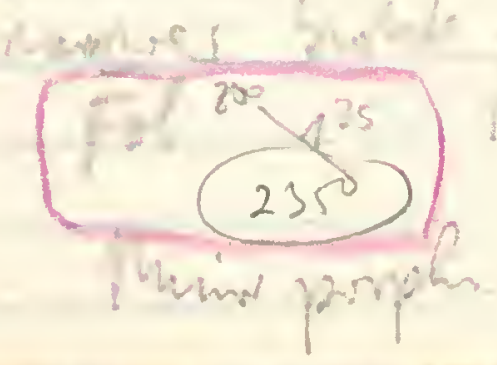




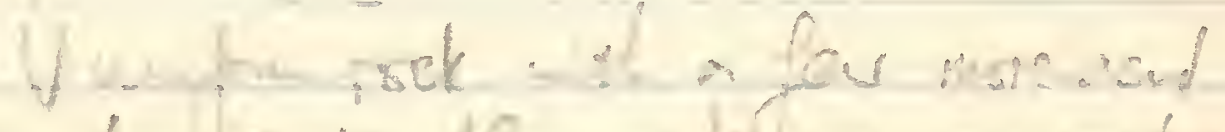

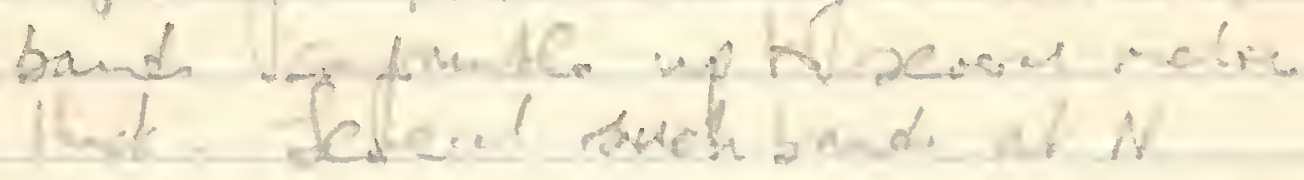

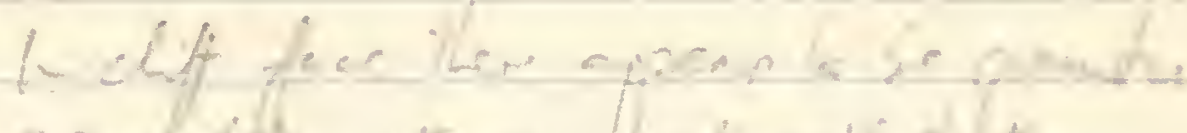

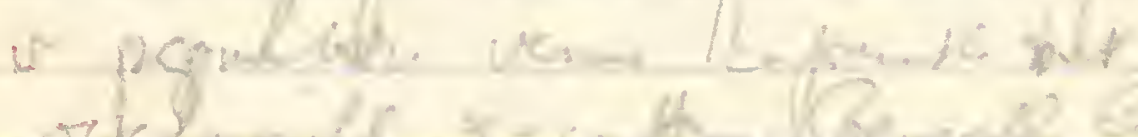

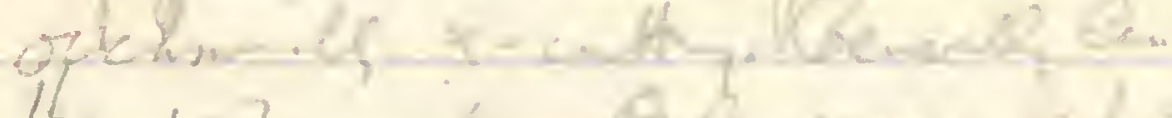

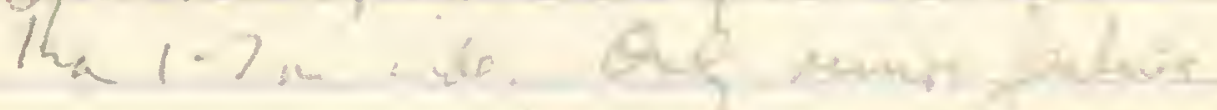

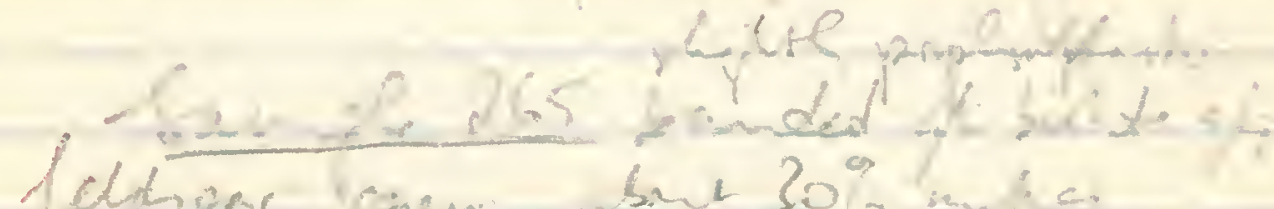

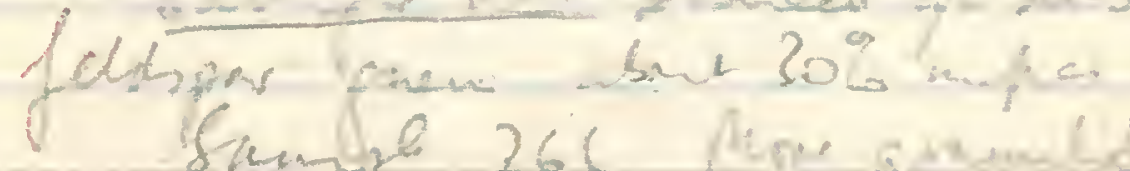

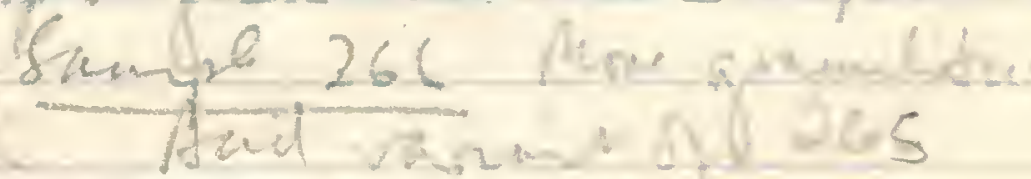
it be ih fest. Gounth

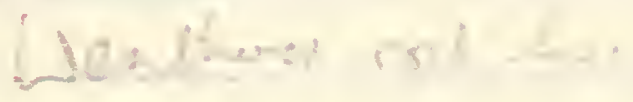




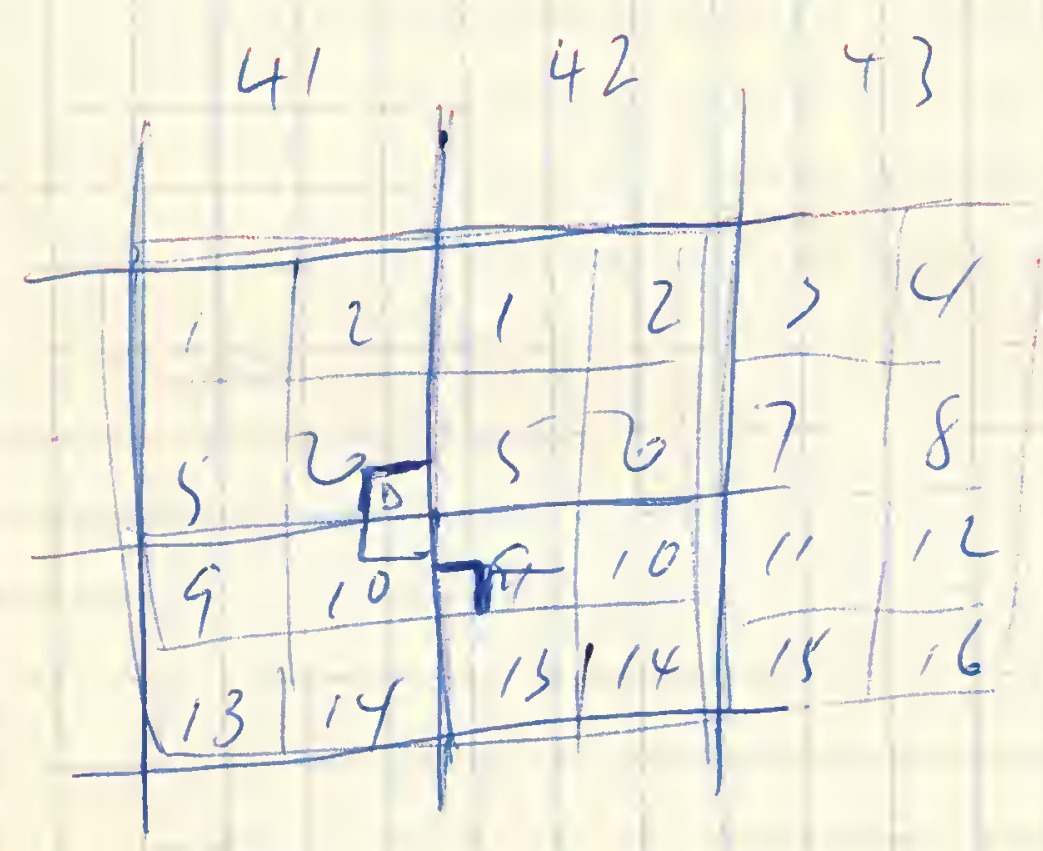




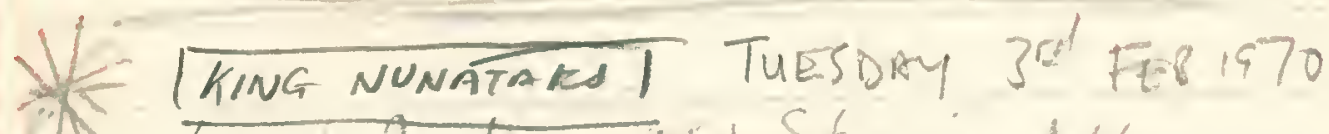

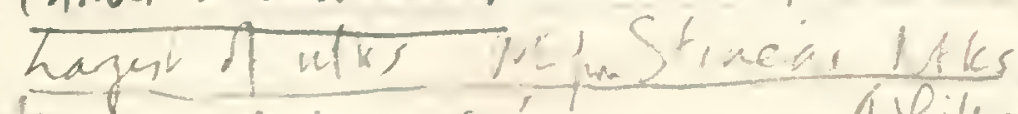

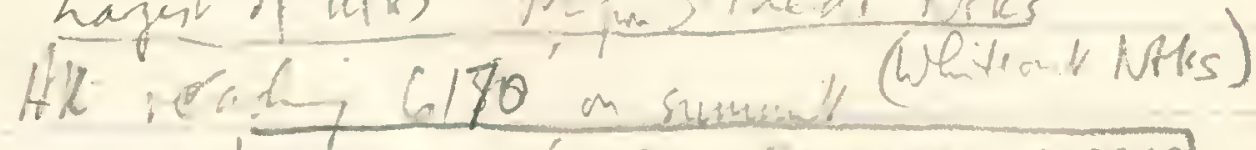
Low 267) Megmith

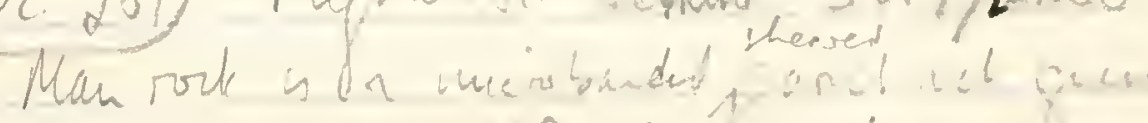

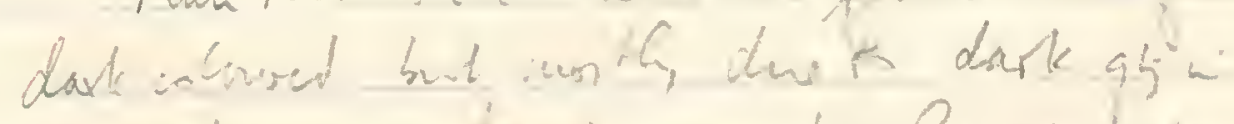

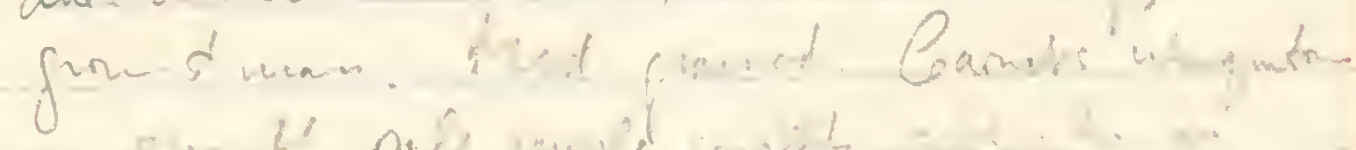

paphes $=$ Clots ad lewn?

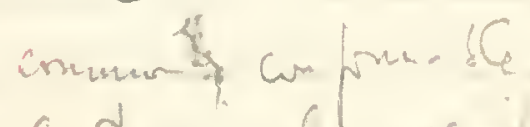
and requalit res munite nater. 
Leyli io: Ua for Cager acid vem

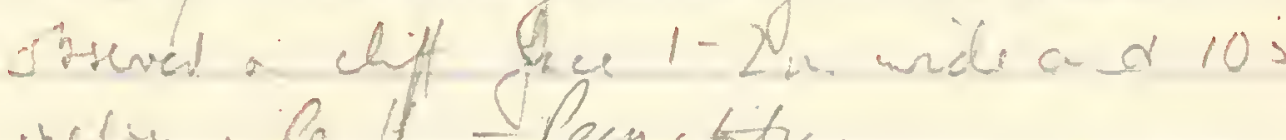

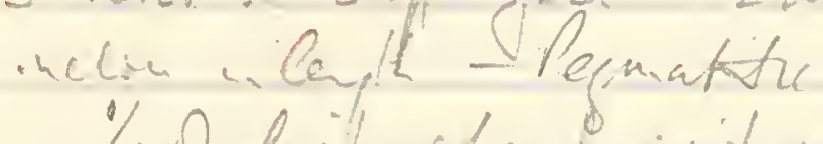

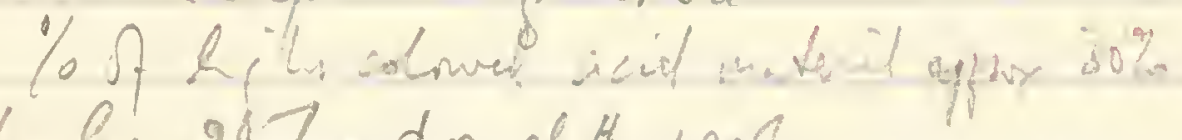
at $b_{x} 247$ ad or chpt near.

Fol. $p$ so

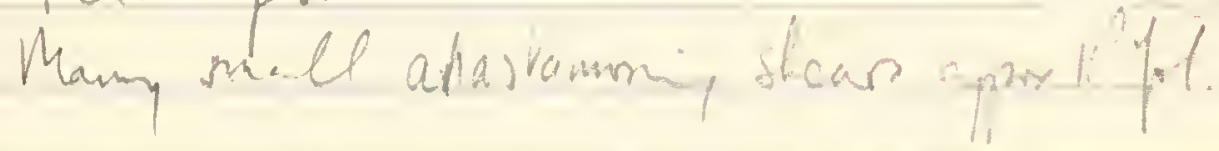

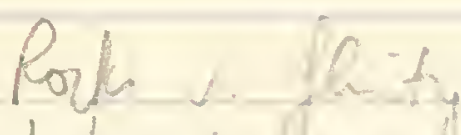

Intan.?

untith cad

Sasy yec

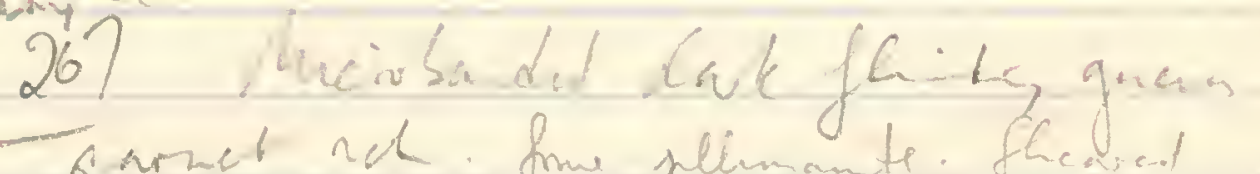

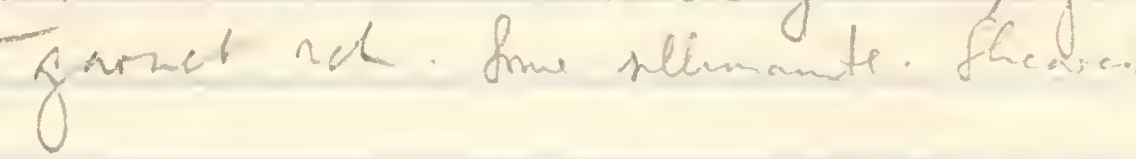

268) fouge phem.

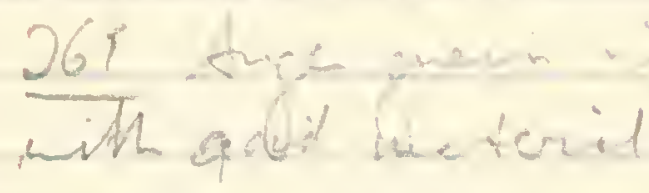

$\stackrel{+}{\omega}$ 


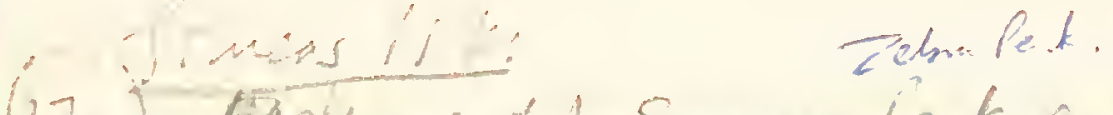

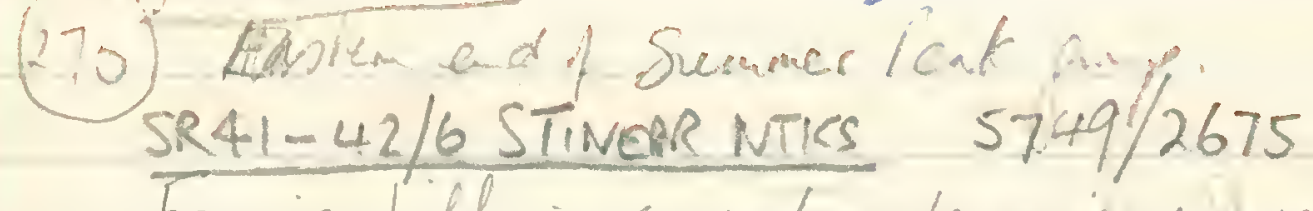
Frmair bill is an a himef

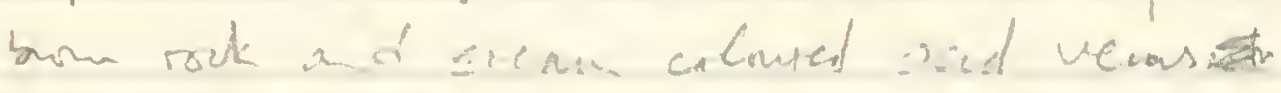

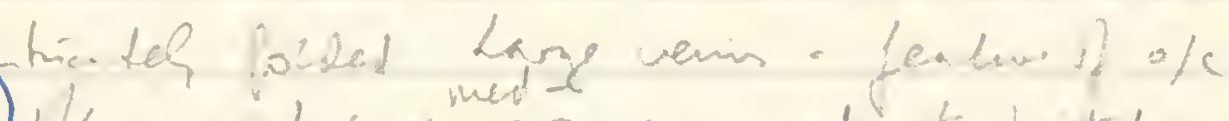

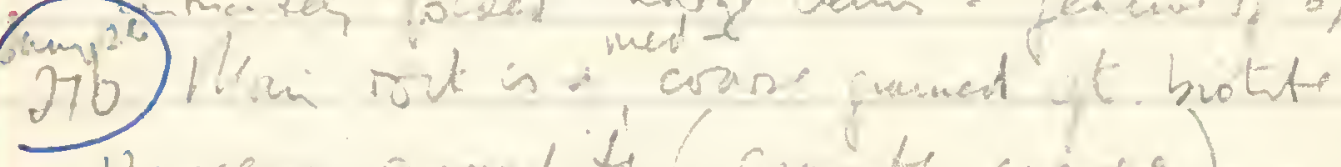

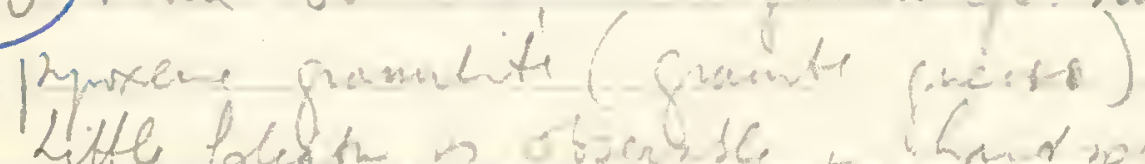

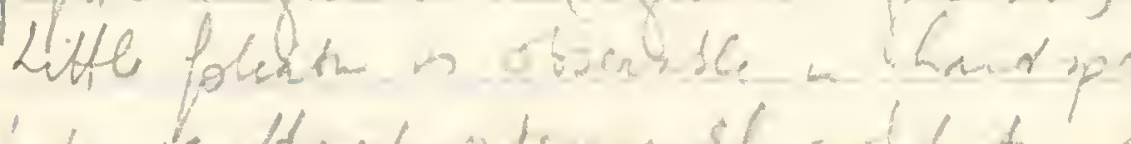

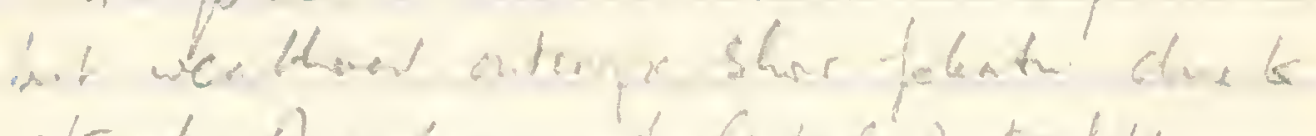

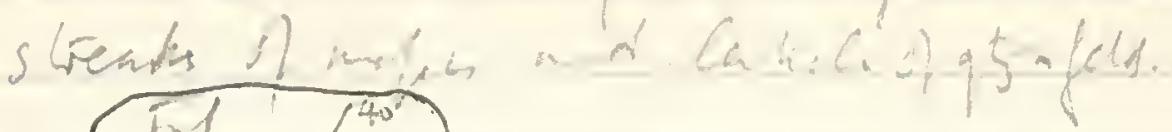

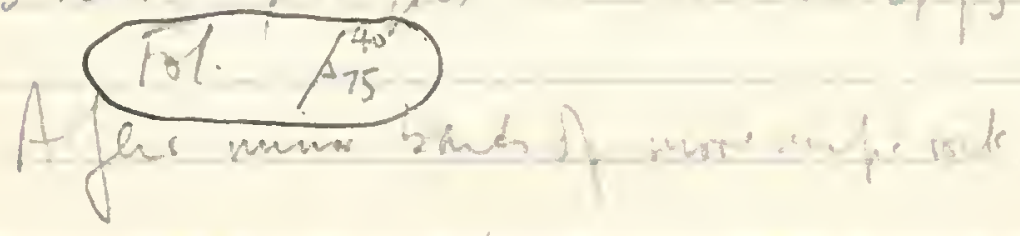

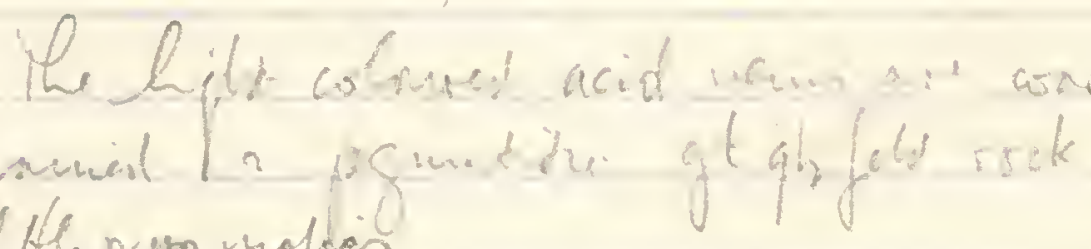

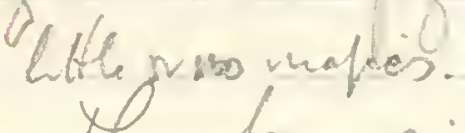

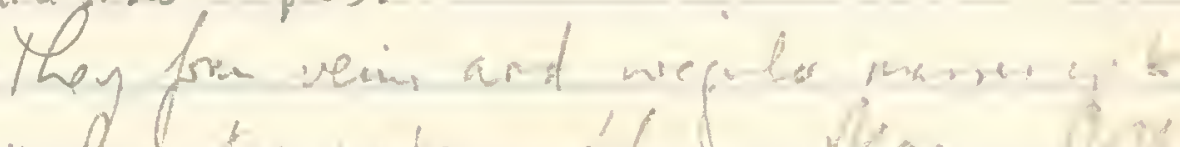
$10, \mu_{1}+x^{2}$ Eazer are coltor simg है: then kancles acid

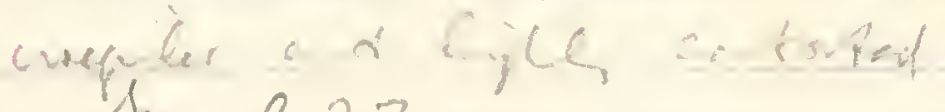
Soungle 271 


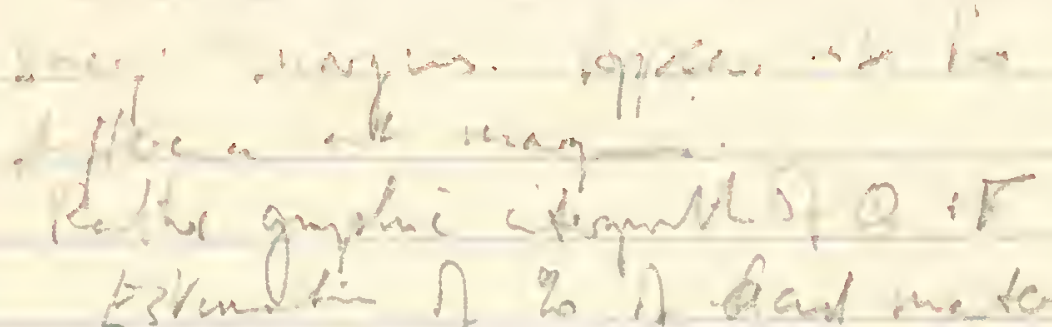

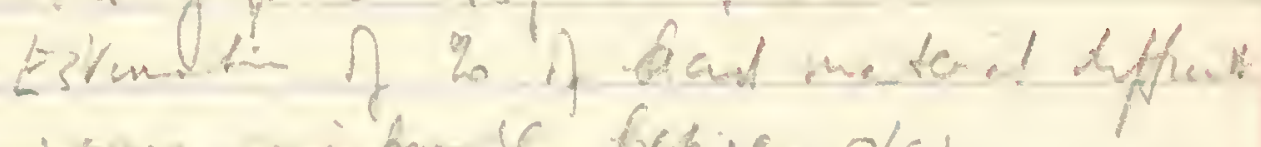

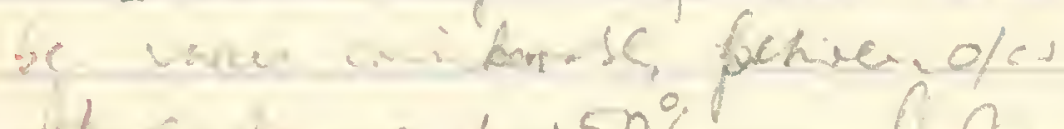
ill chices cs $+50 \%$ Then Near cetir len th. 30? Everal Garg aren with a remp.

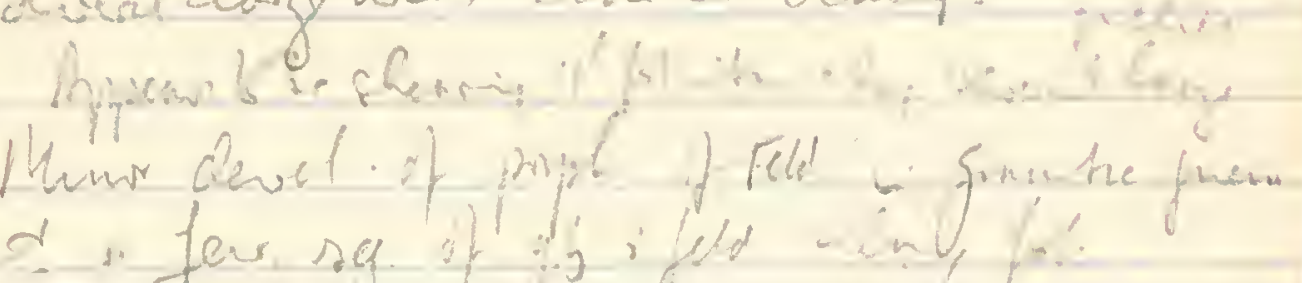

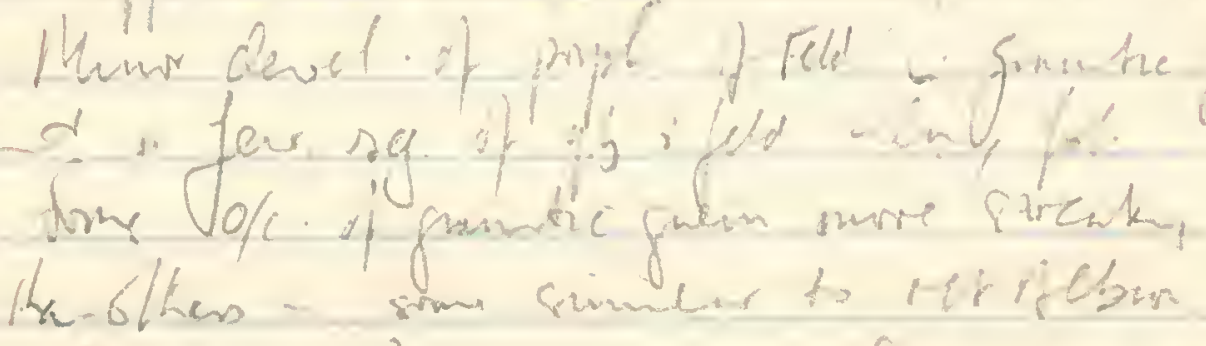

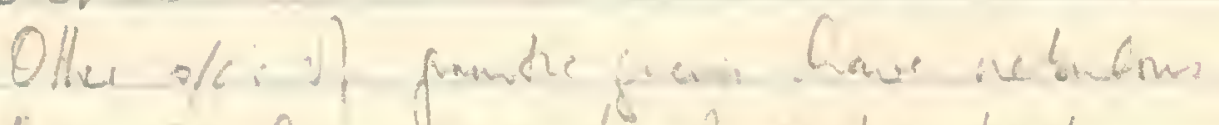

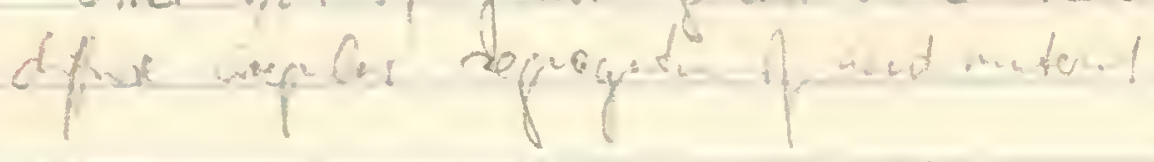

Fenliw ! these hith or th largste? verm lifs colowel

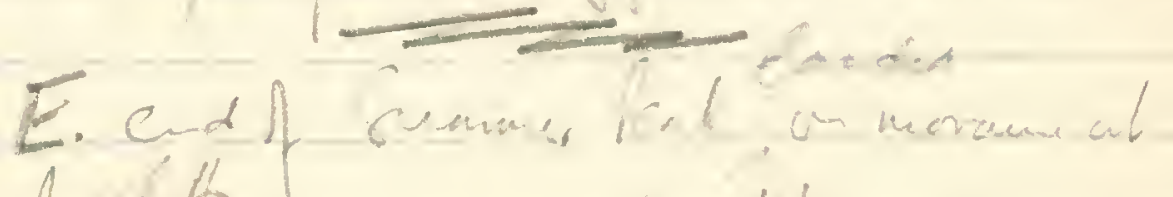

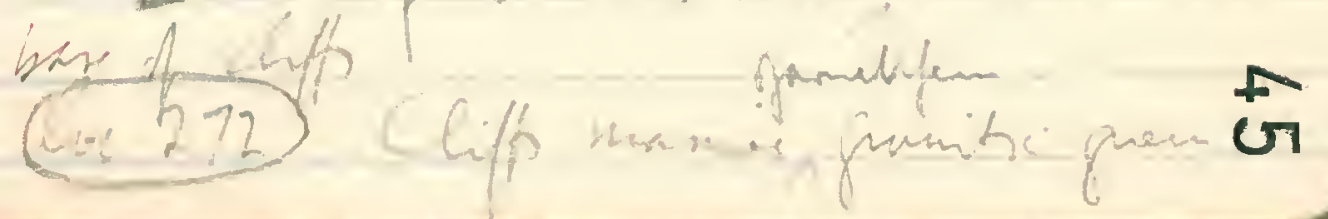




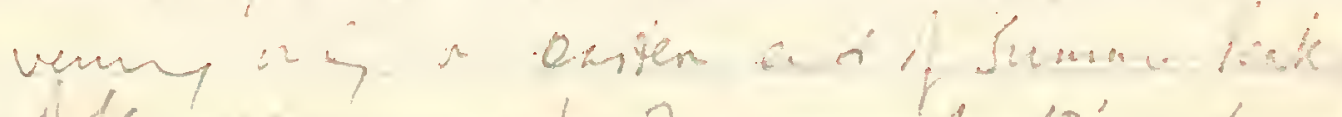

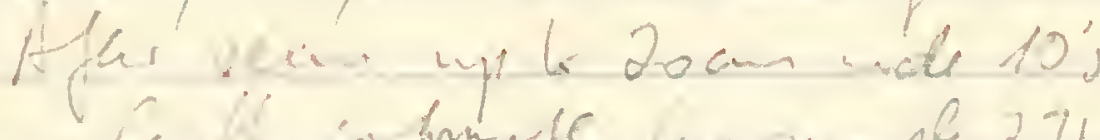

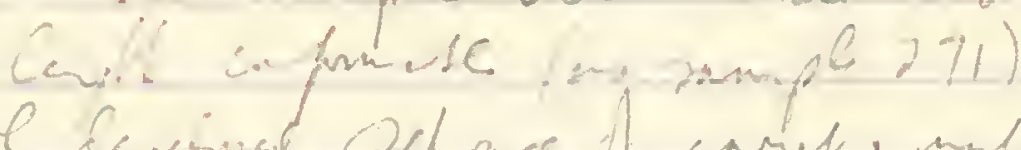

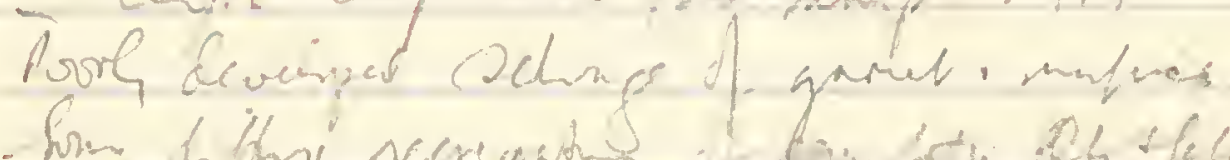

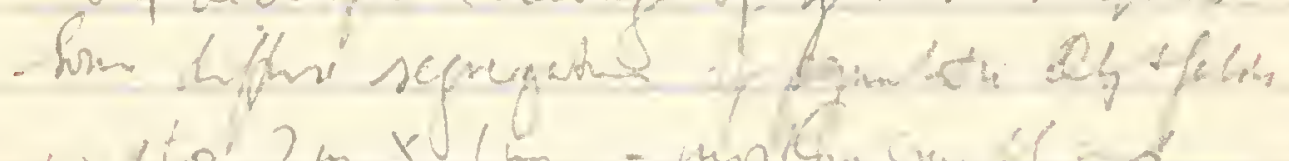

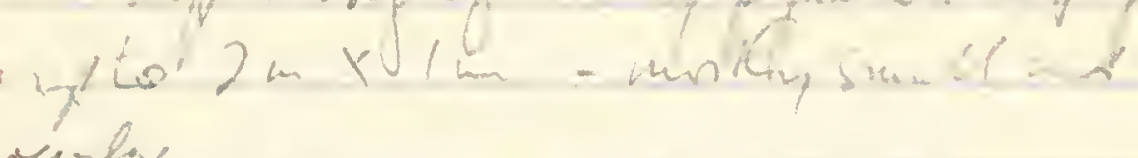

uryelas

Hecuir lab bad chave! toff

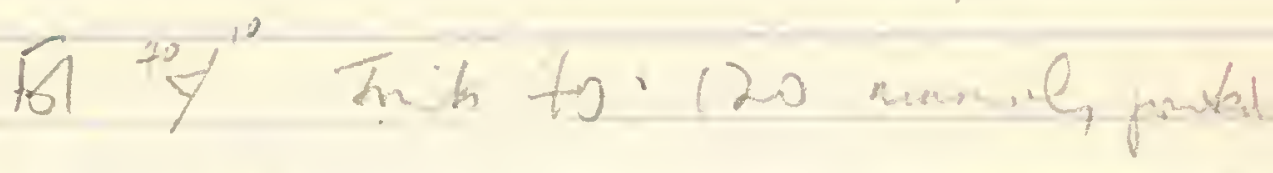

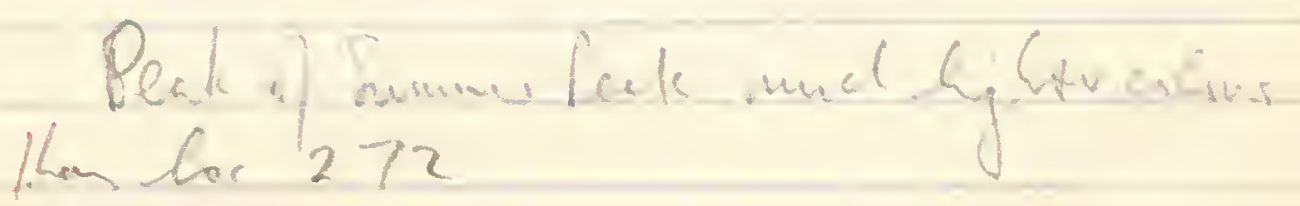

SR 41-42/6D STINEM 1/4t.

$5731 / 2667$

के 
Loc 27

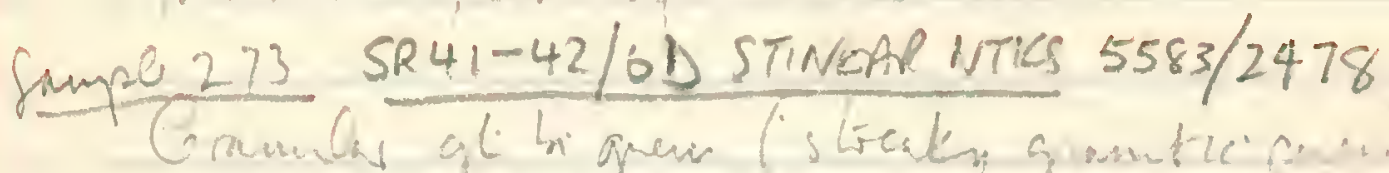

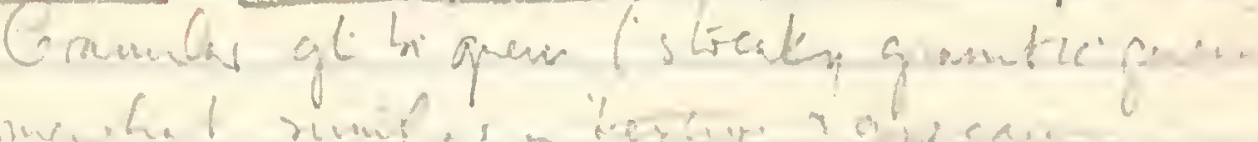
is 210 a d at Somm tot med proment.

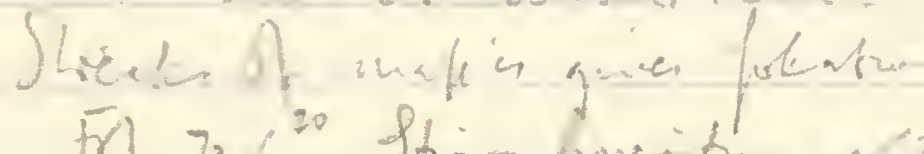

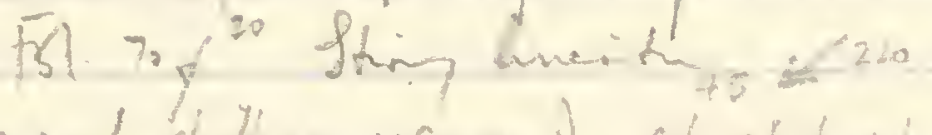

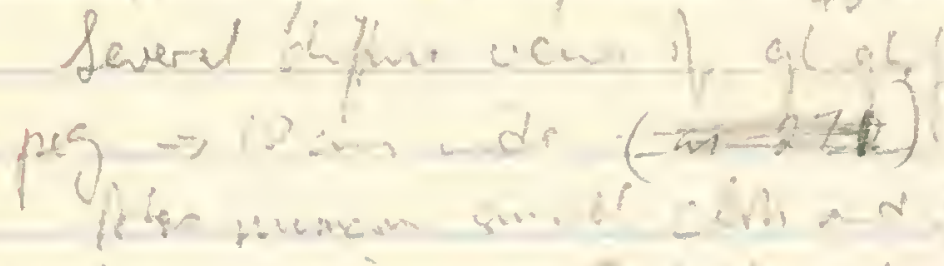
meples. But u Jew drevet and veling; $k$ Oic: how a nebethr

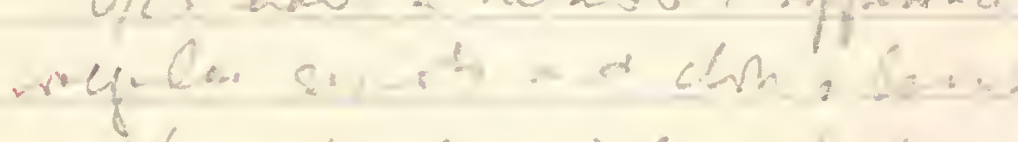

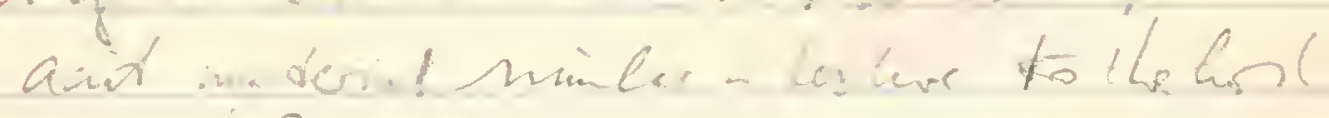

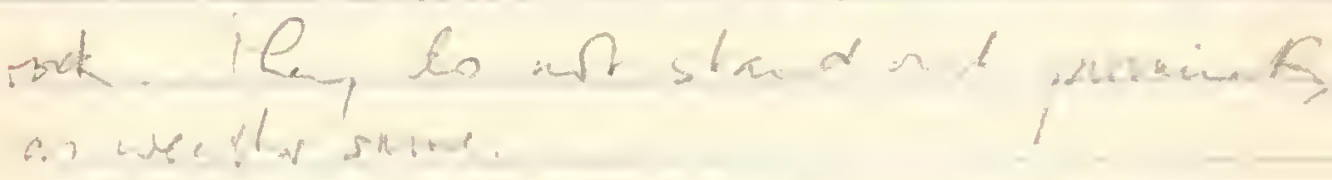


Rilide /Ke $\frac{5 R 41-42 / 6 c}{\text { Landivane wTSS }}$

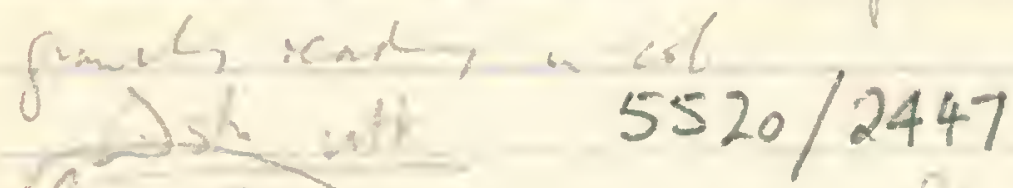

(Coc. 274)

$\operatorname{limpl} 274$

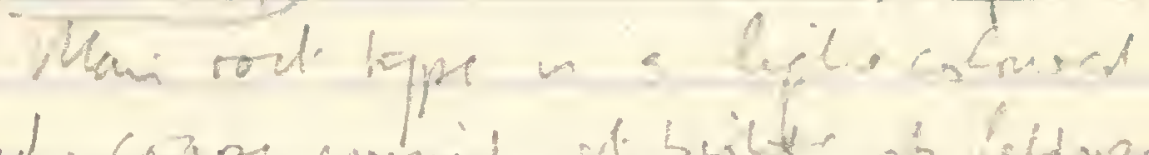

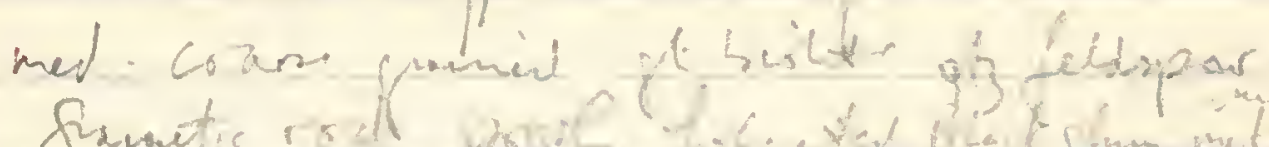

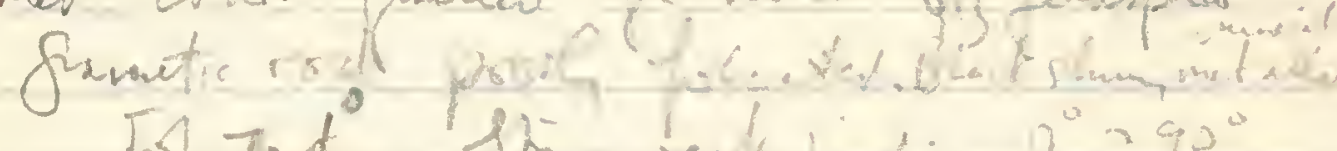

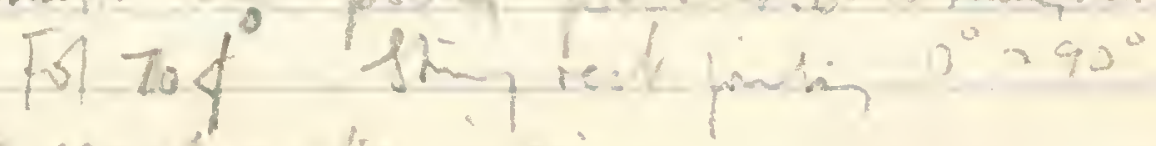

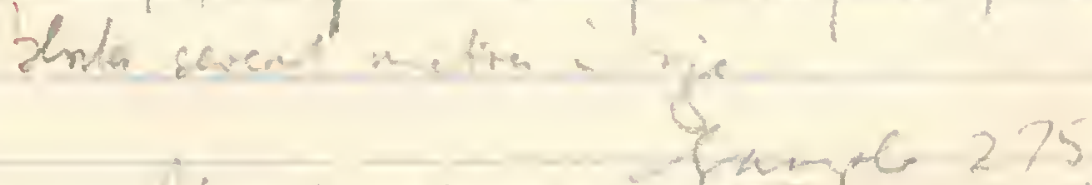

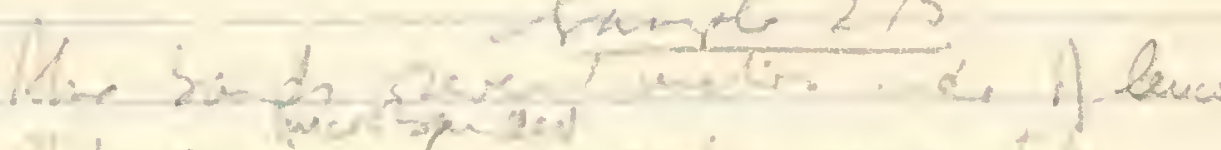

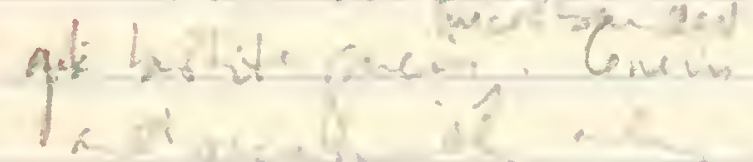

Contrinis ploph

siagres

t. 10 romed

fout. 
ens $x-c_{n} k^{\prime}$, pron ven: $x$ and

th ofwhegrem

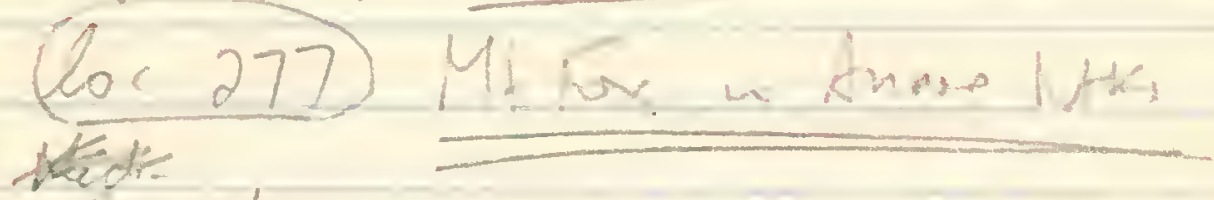

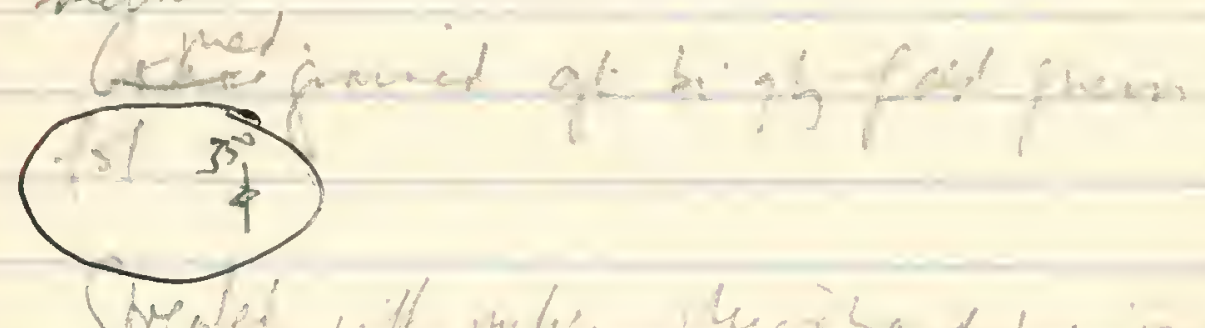

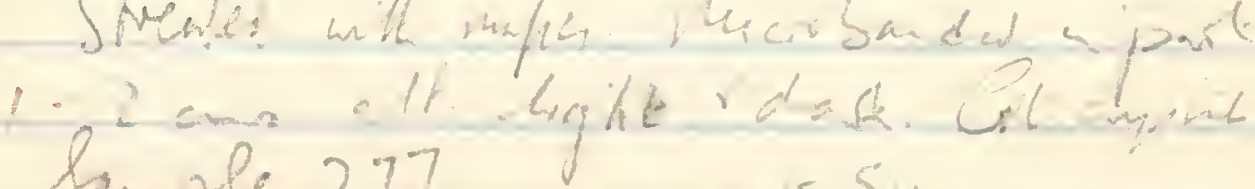

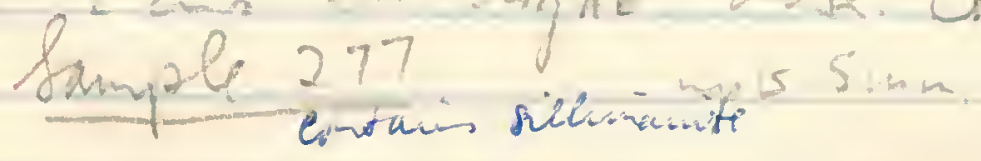

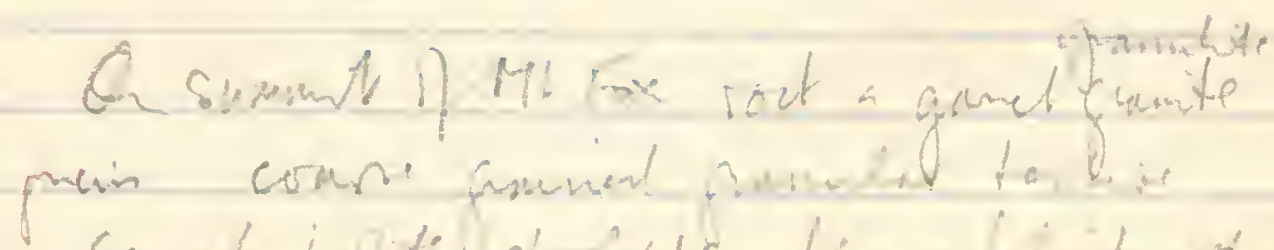

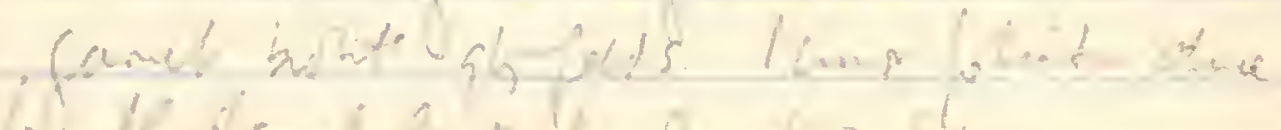

Sample $278 \quad 51 \cdot 10^{\circ}$ vel $\left(80^{\circ}-1\right)$

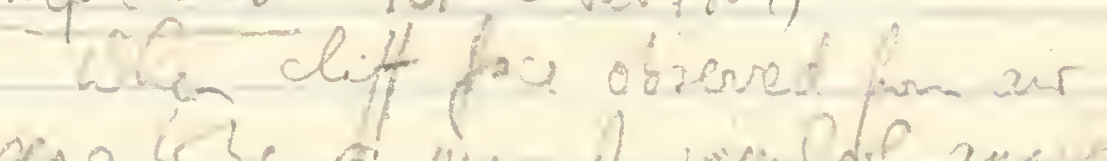

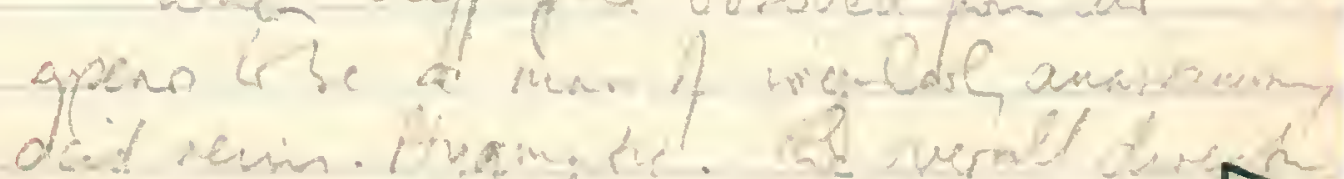

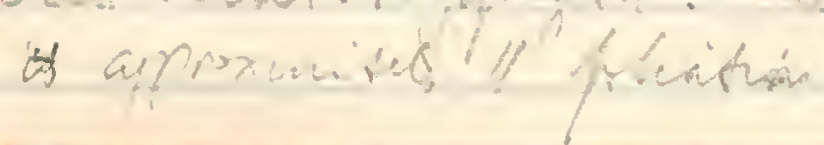


ThUPSDMA $5 \overline{5 \sqrt{\text { FEB }} 1970}$

Hee ploso Antay

Himeng Peaks. Mt Scabor or $1100,0005 R+2+43 / 9 C$ RENEY LARE

Camp sute a cerque MW If Sentor

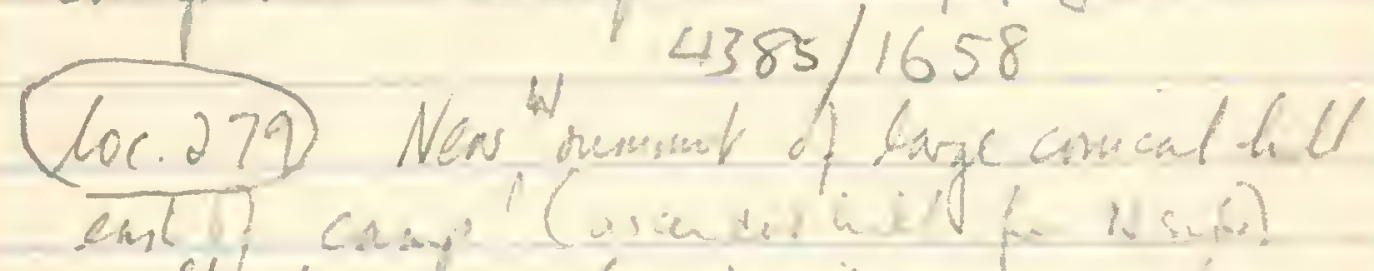

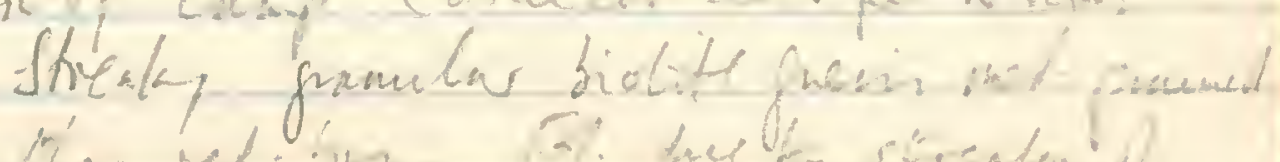
we then net óno

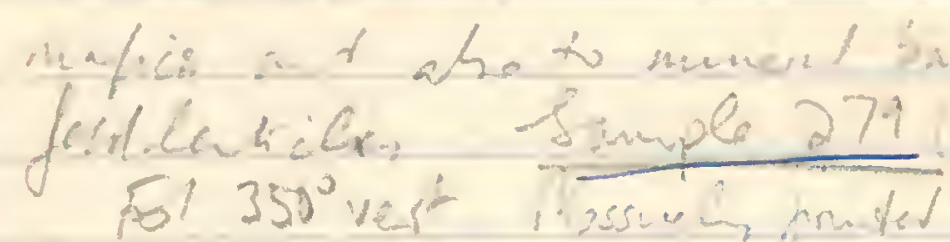

$\angle 718\}$ 


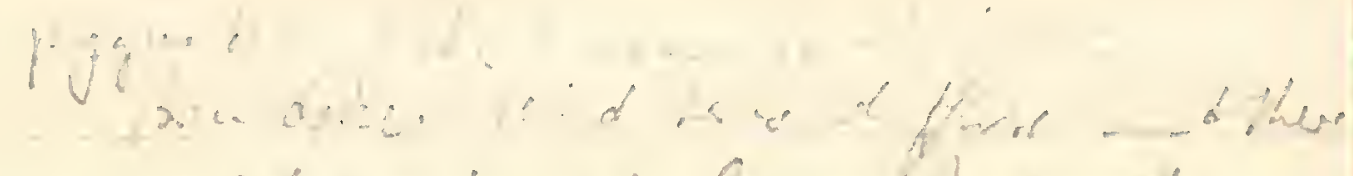

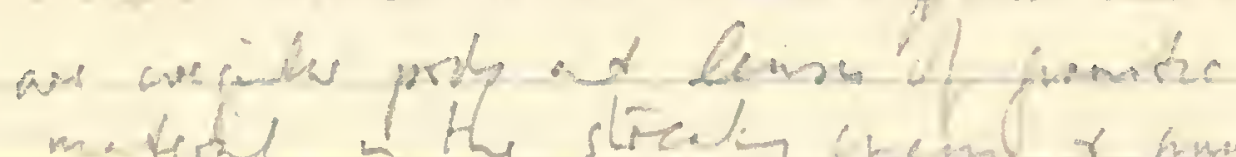

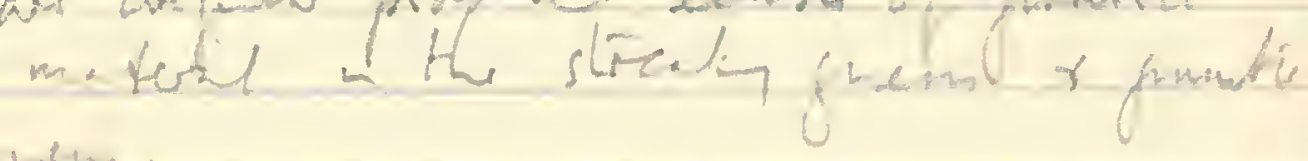
Q.nethe

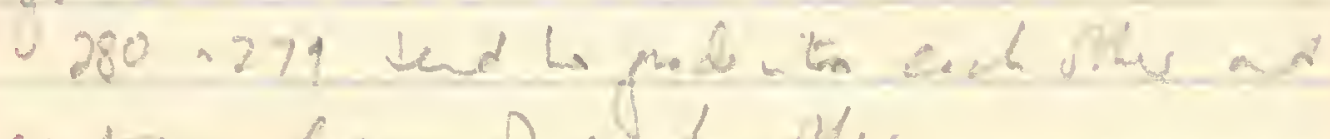
andrua leaven of

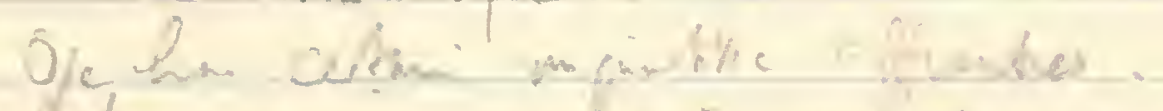

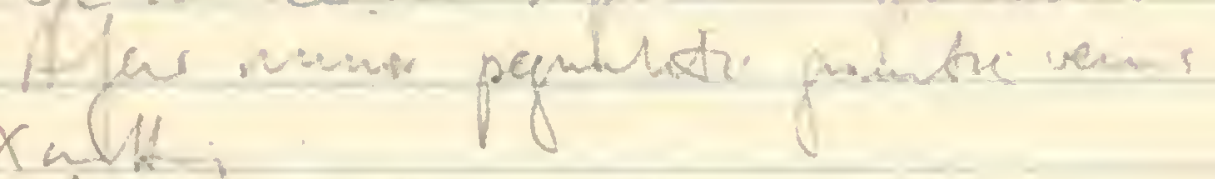

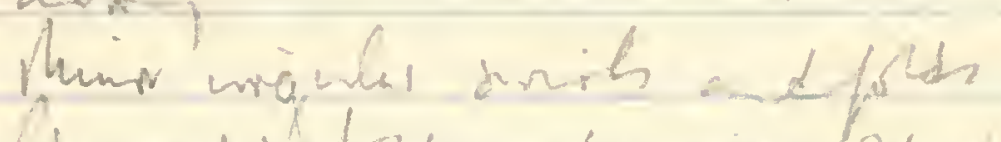

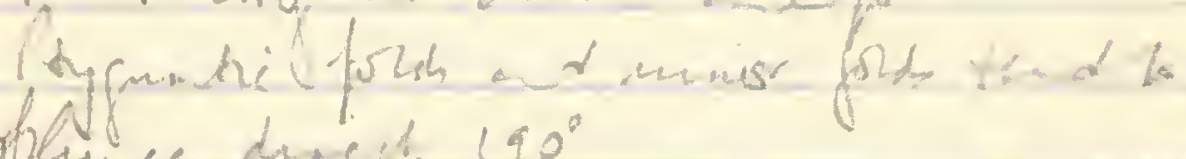

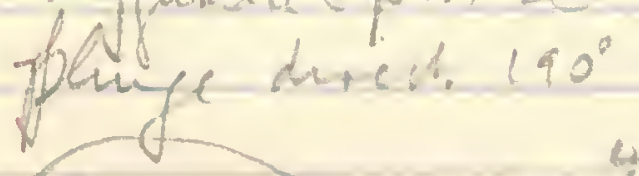

(lac 282 $4387 / 1659$

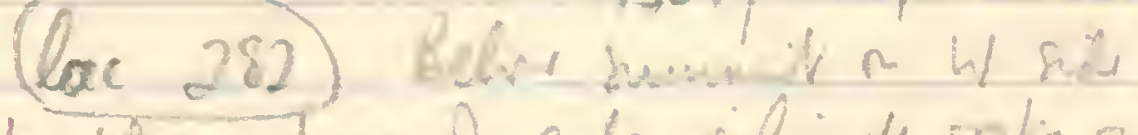

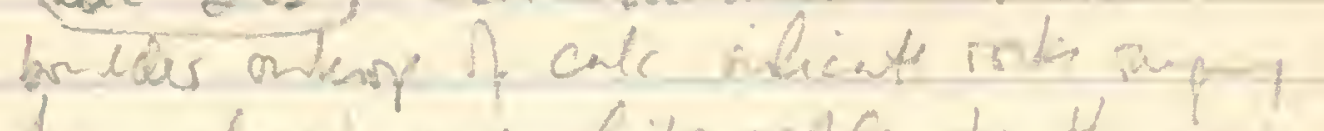

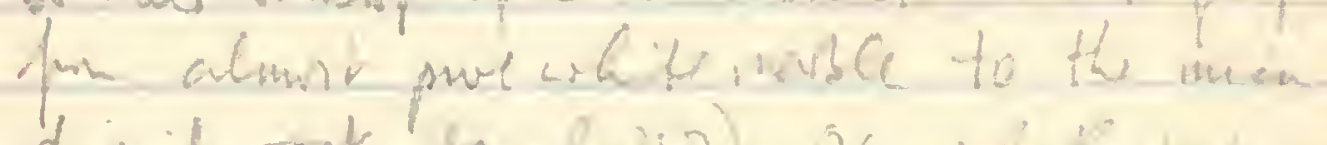

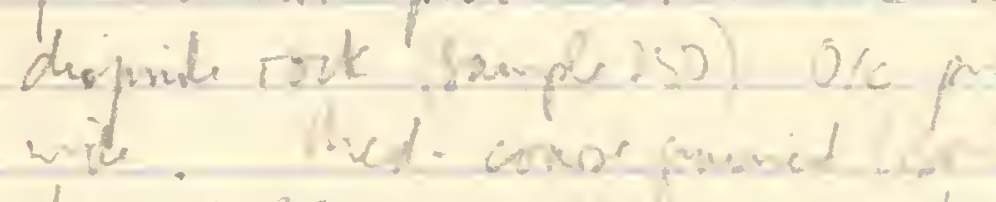

templ 282 mabe

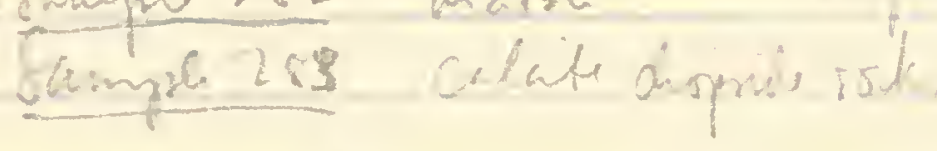

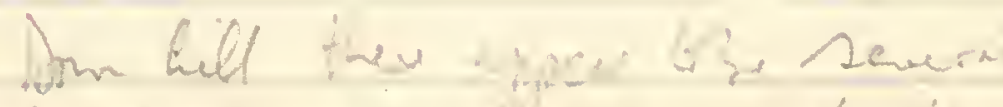

i) dien rovk a th 


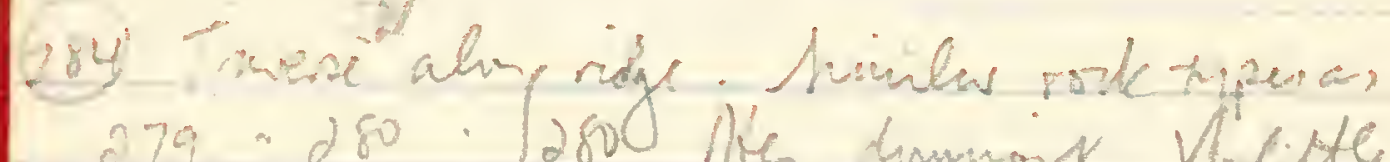
279 -280; $2800 \mathrm{gh}$ dummin Vlecte $\operatorname{lin} 1: \mathrm{i}$ ank

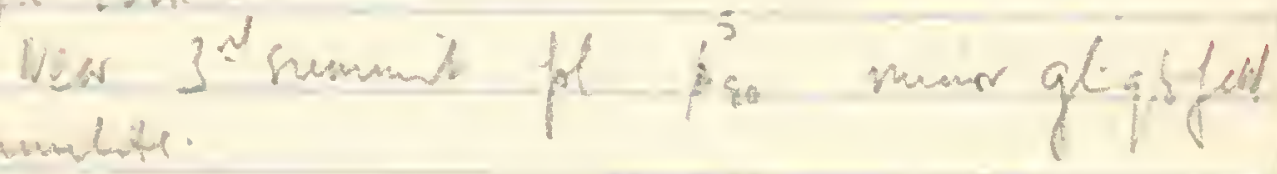

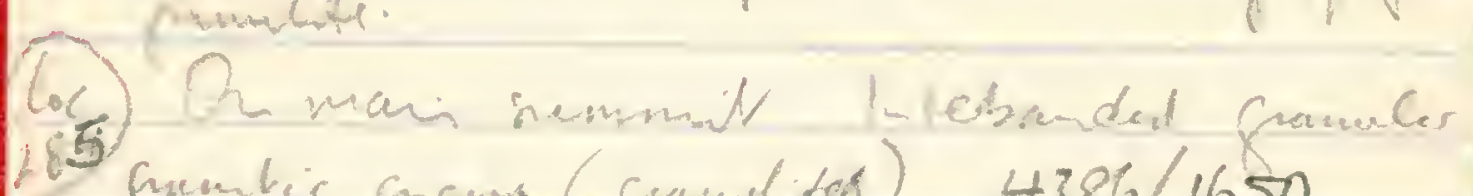

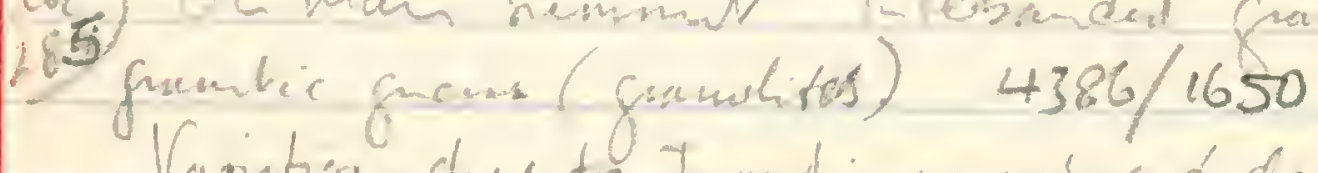

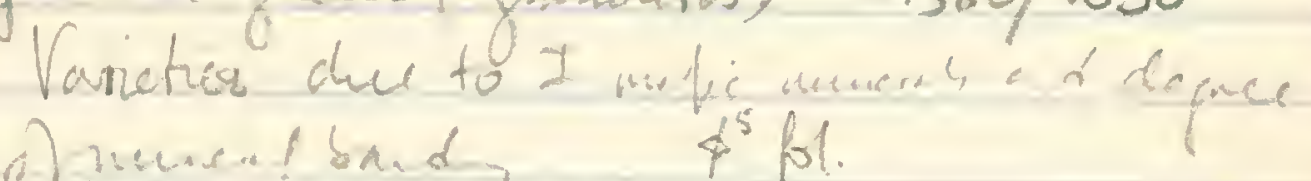

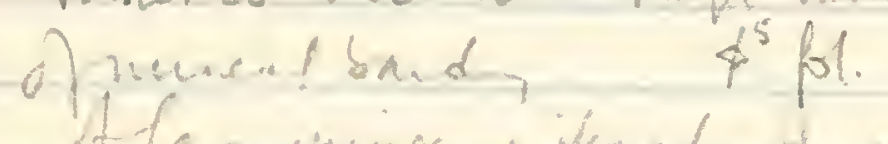

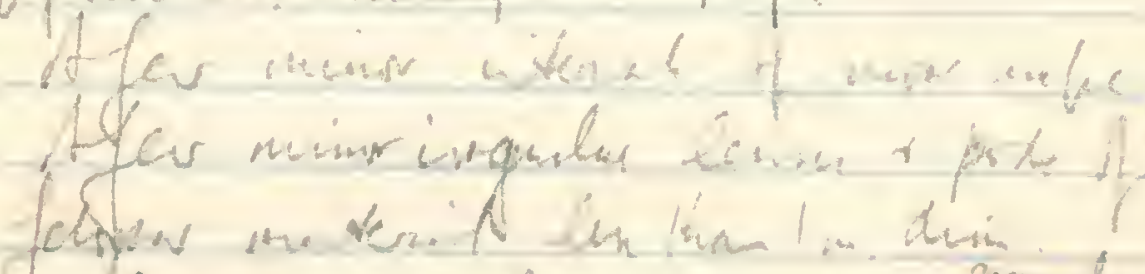

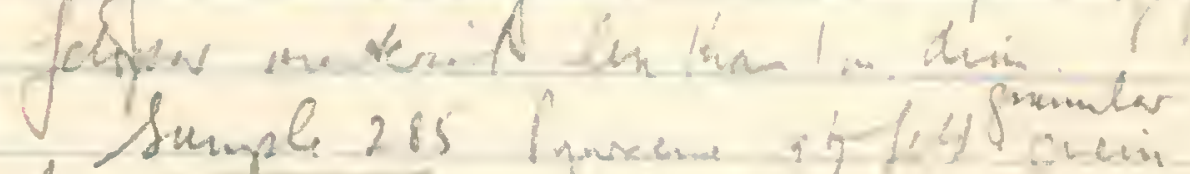

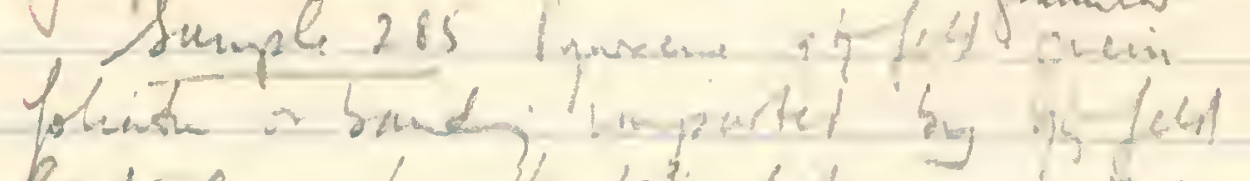

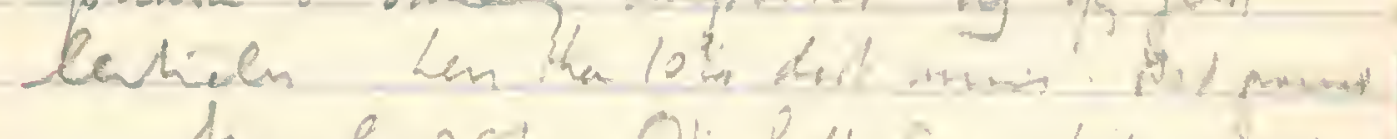

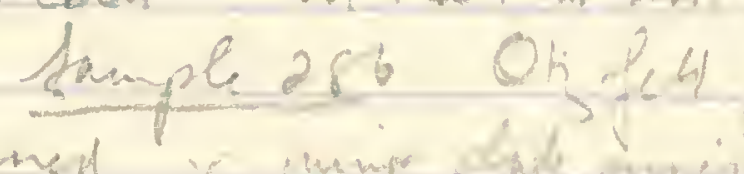
gramed mis bet ix

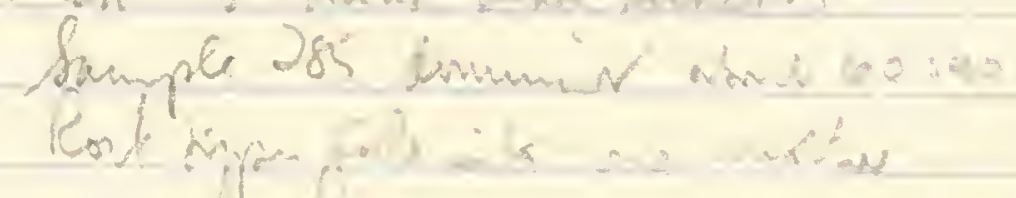

(Romat it plisurs 


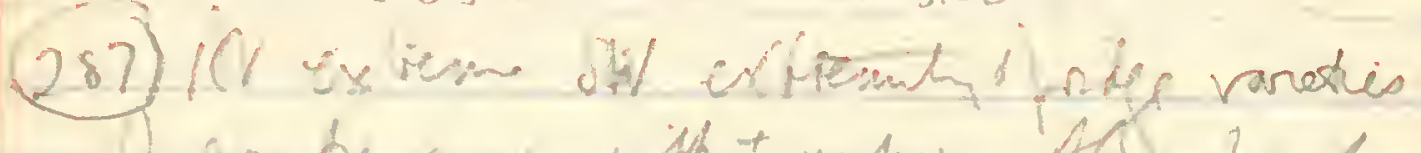

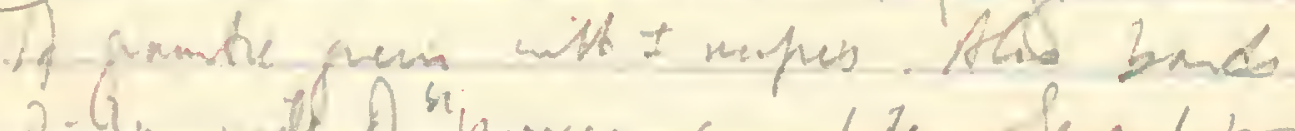

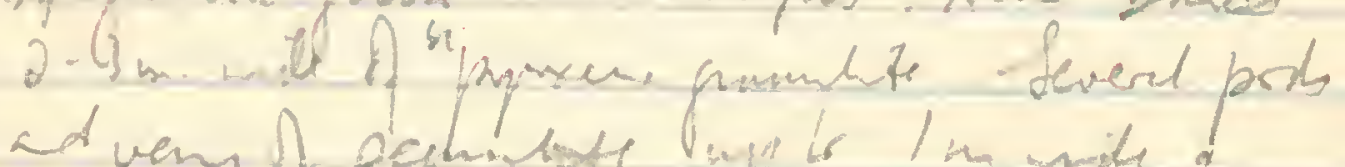

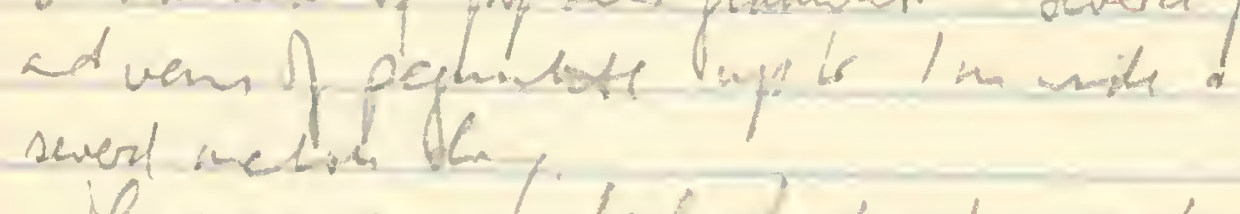

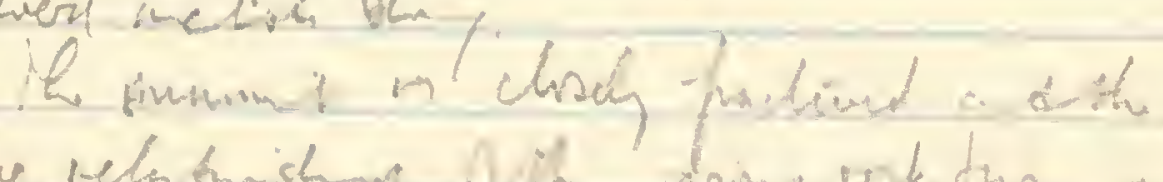

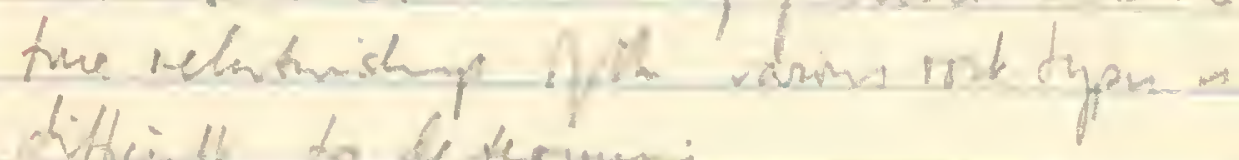

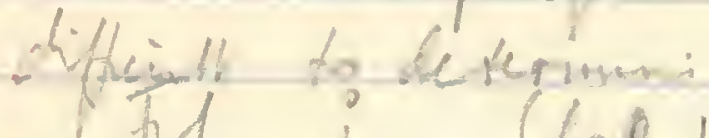

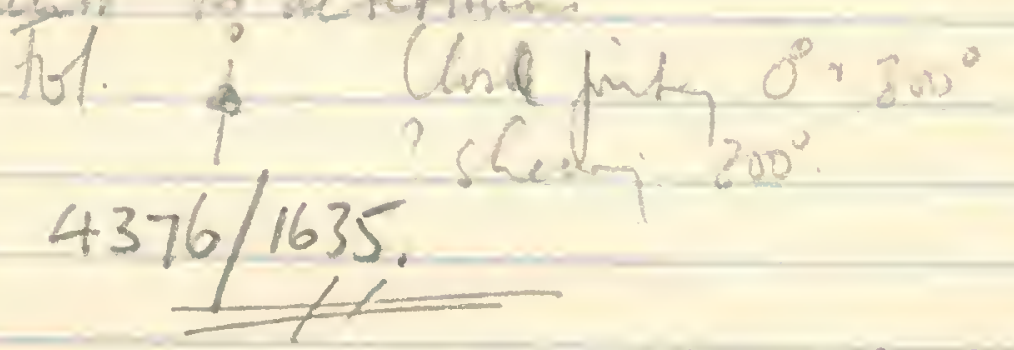

Or rige top reweral aren of tors suth

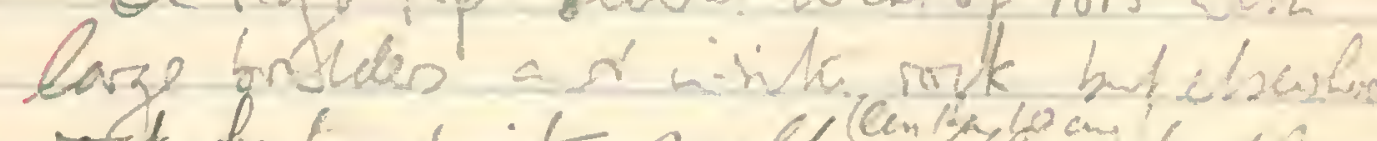

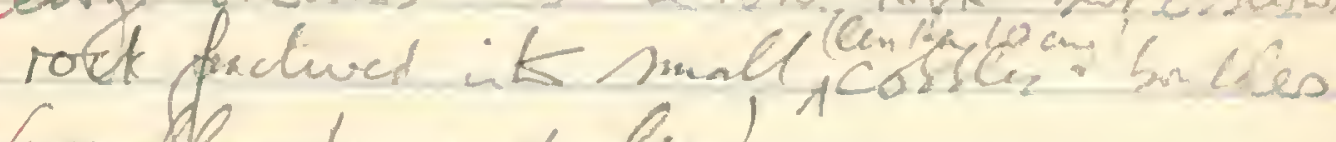
crmally, Im ad len!

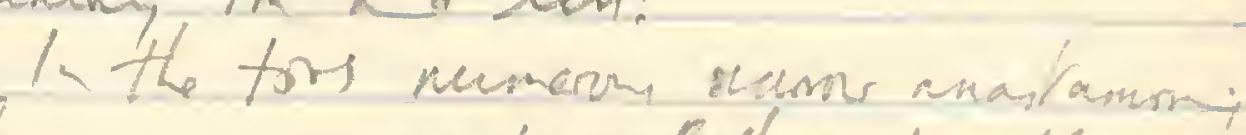

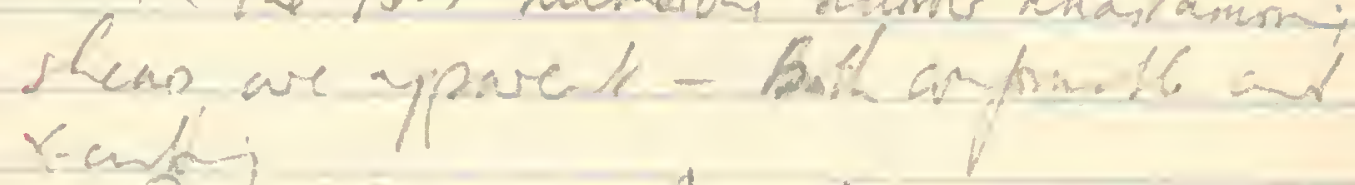
Occarmat zon of schition mafic ook anformatle

$\omega$ 


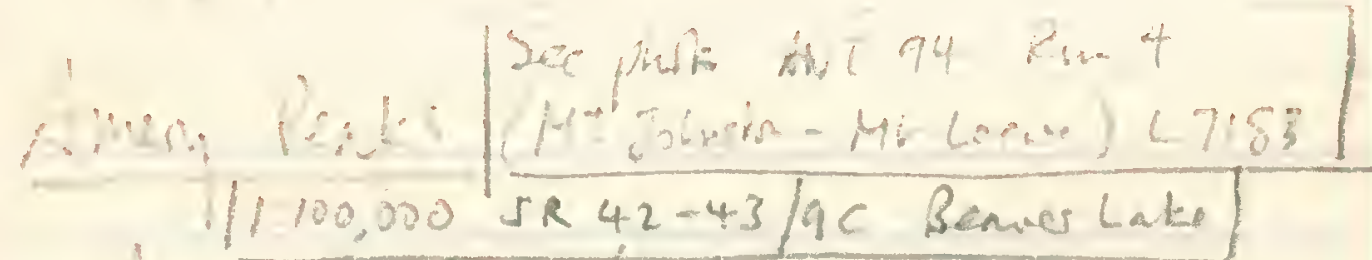

$$
\begin{aligned}
& \text { ixe }-3 \text { ine }
\end{aligned}
$$

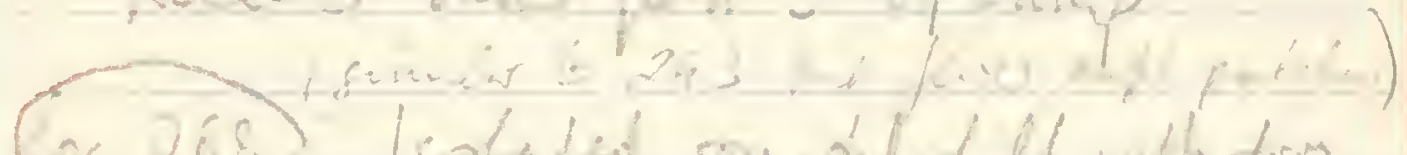

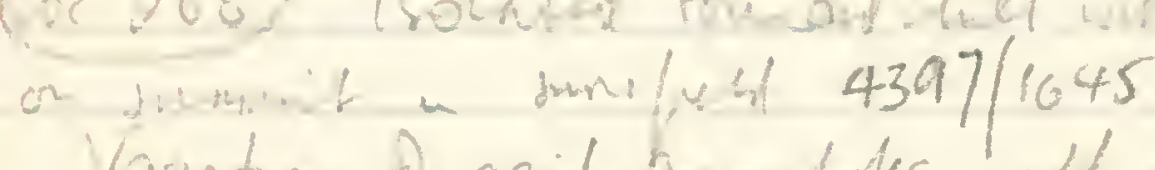

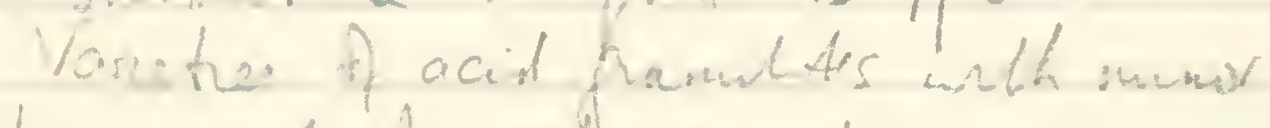

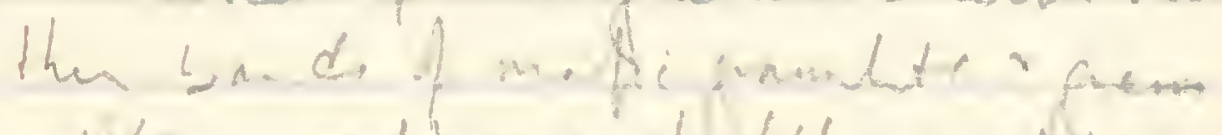

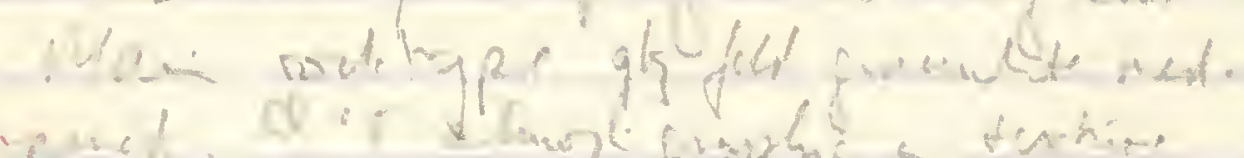

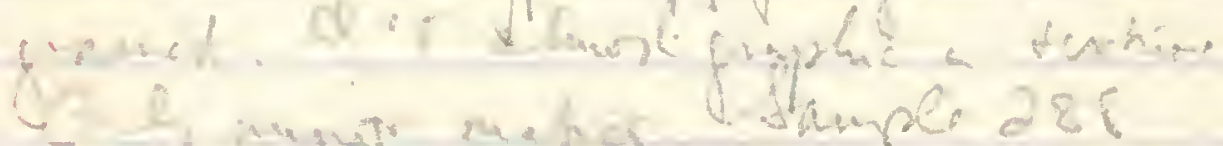

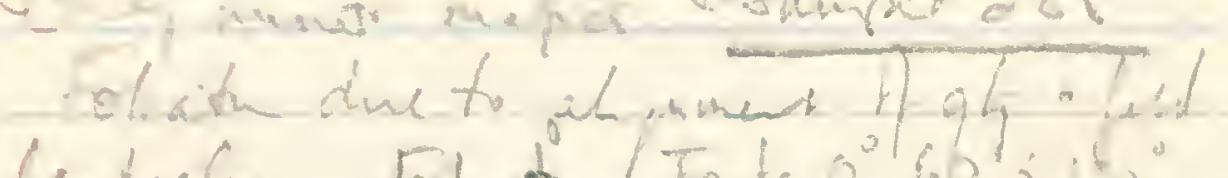

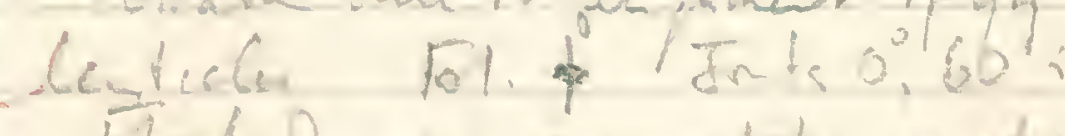

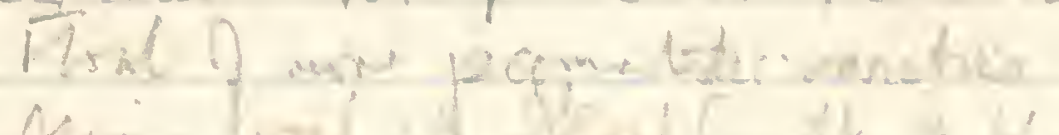
M. $-20.25^{2}$

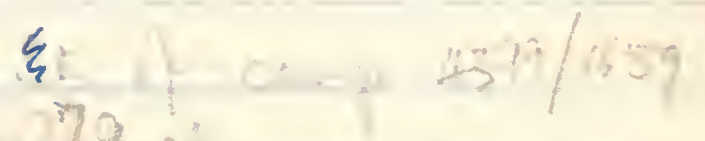
$79=0$ 



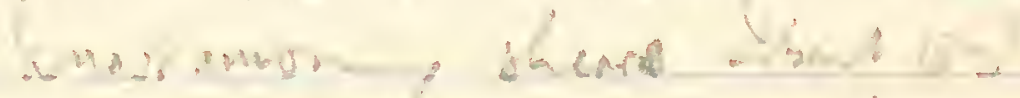

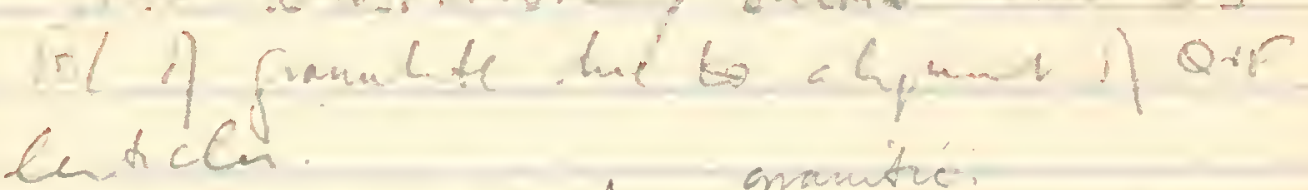

Sample 289 Anid grontic it med armus

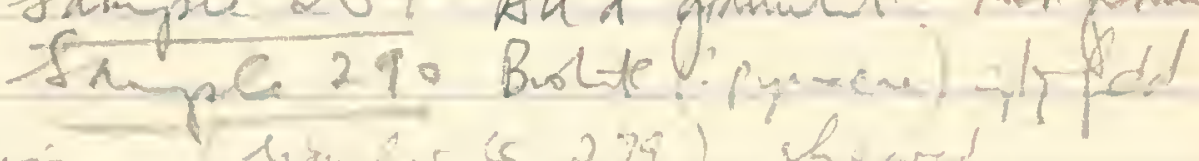

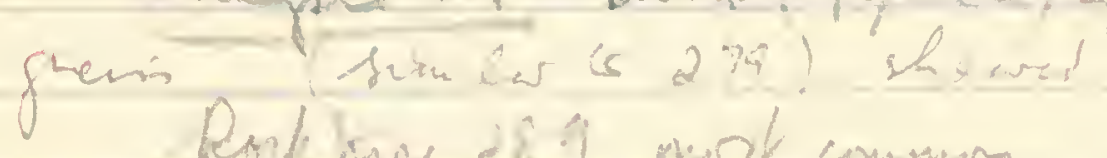

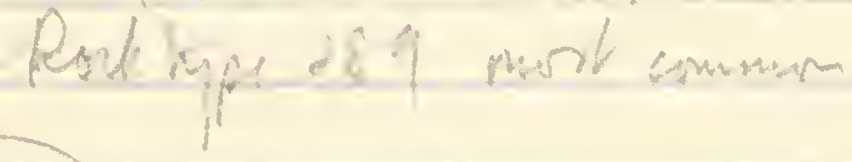

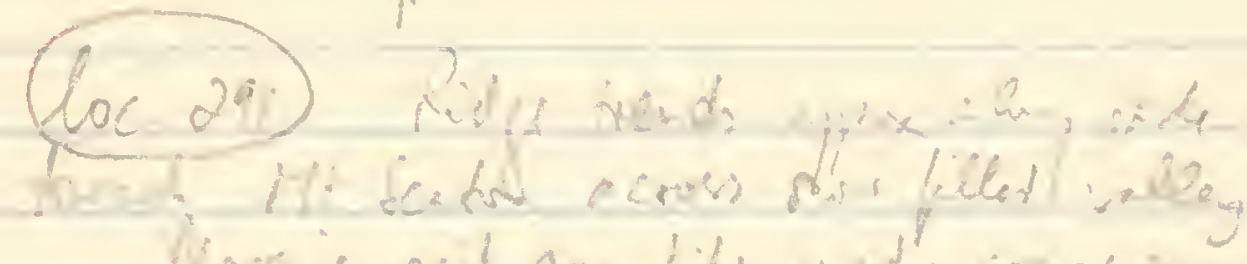

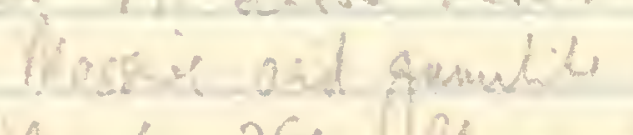

- numilus to 281 Vilu

itho simen

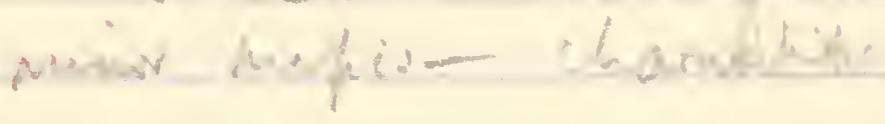

$350+80$

Sinisint $30^{\circ}+70^{\circ}$

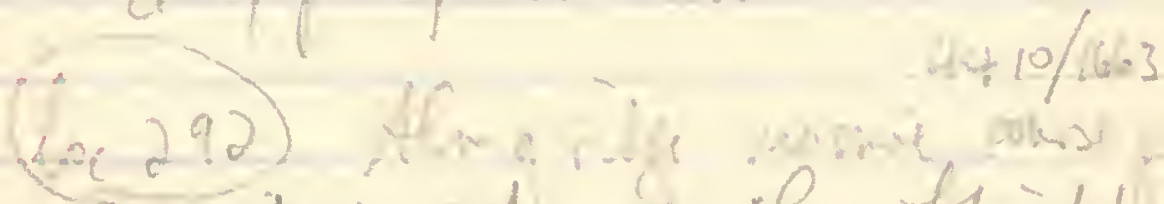

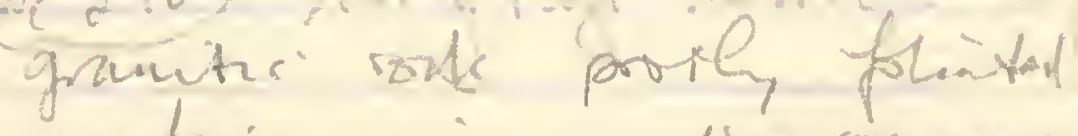

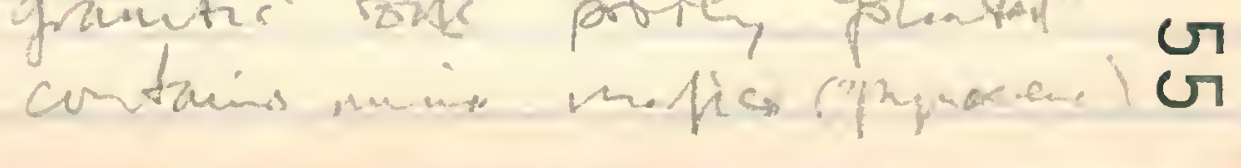




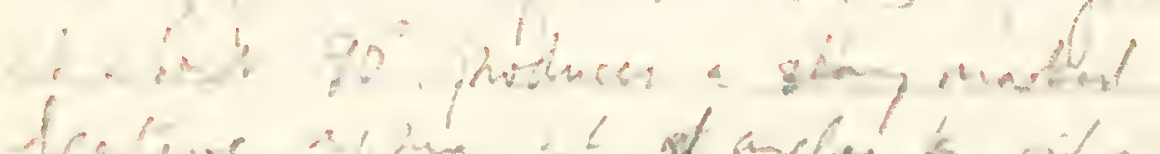

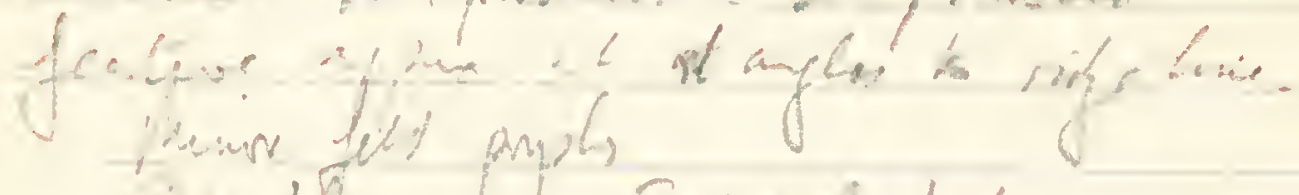
beipl. Fas pal boin

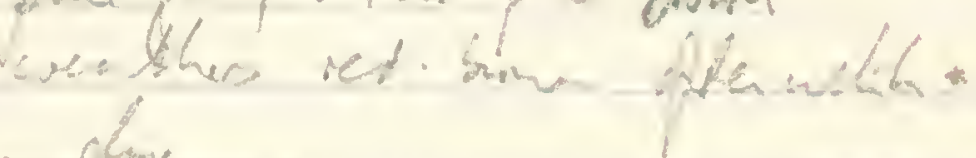

Crantio quara

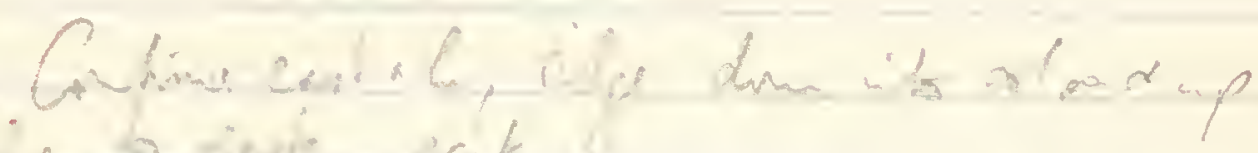
Fith- 6 sintin pak.

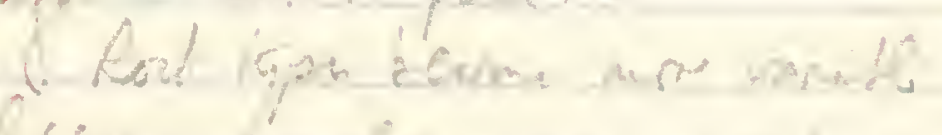

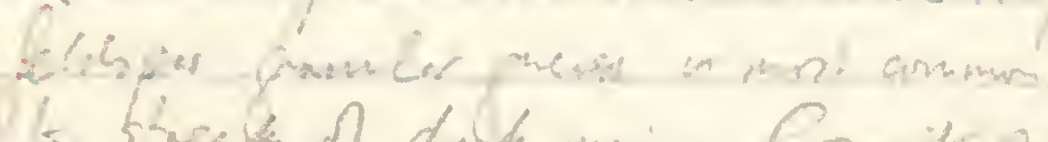

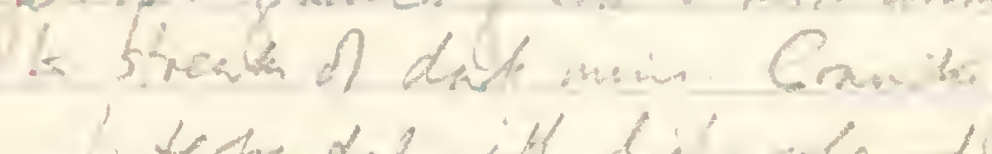

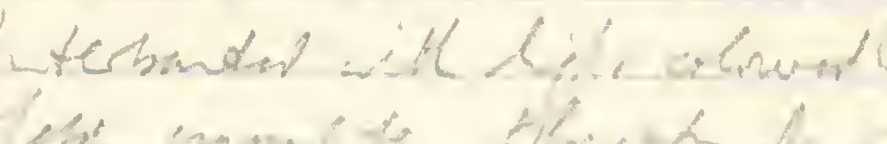

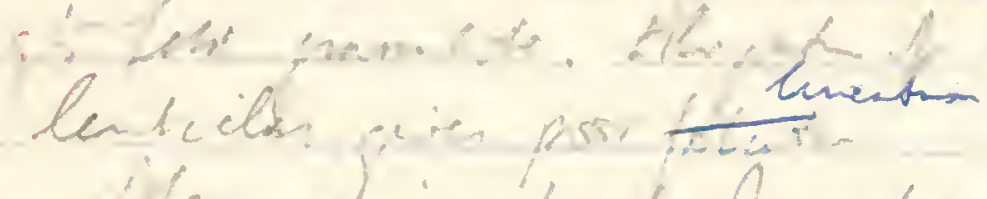

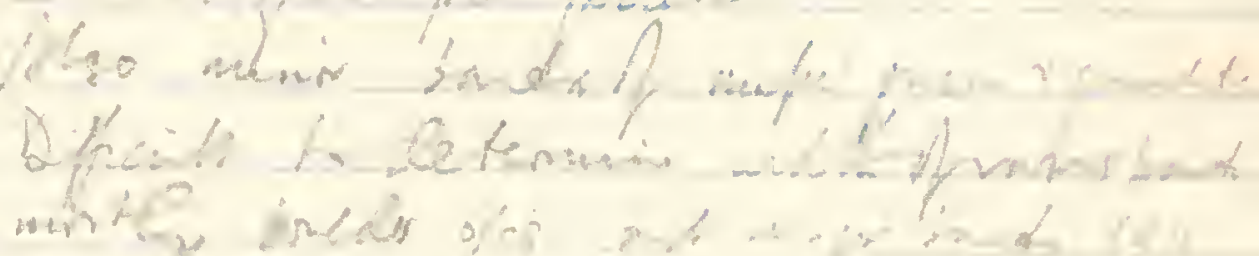

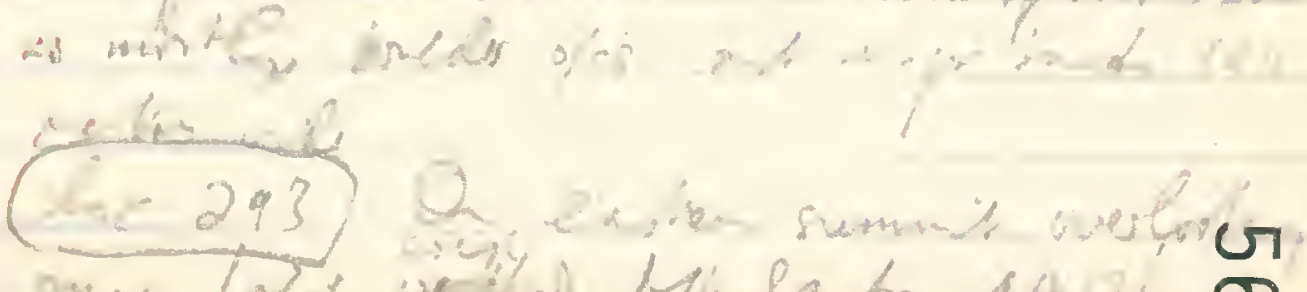

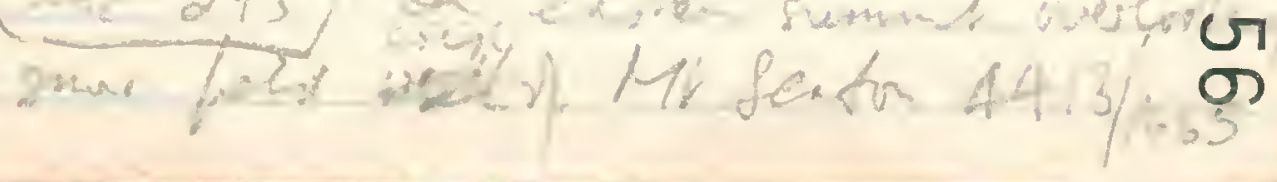




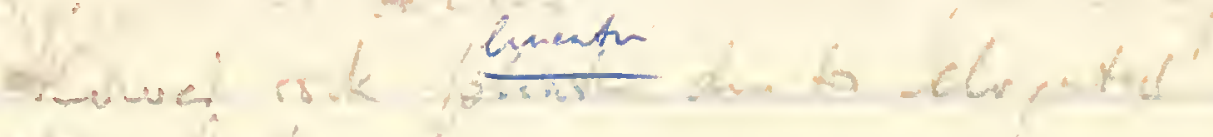

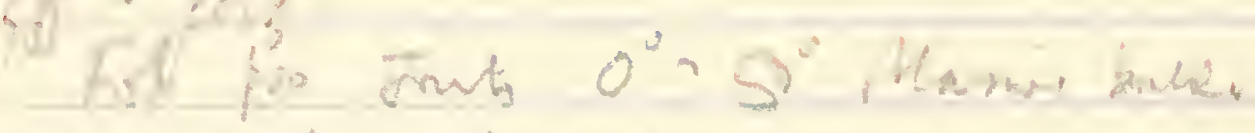
Q.

thar 293 Gt. of fert pimbte

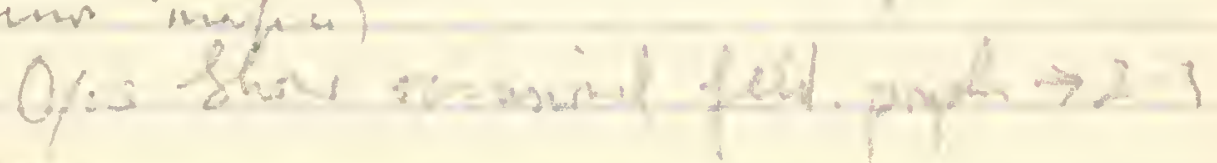
onn

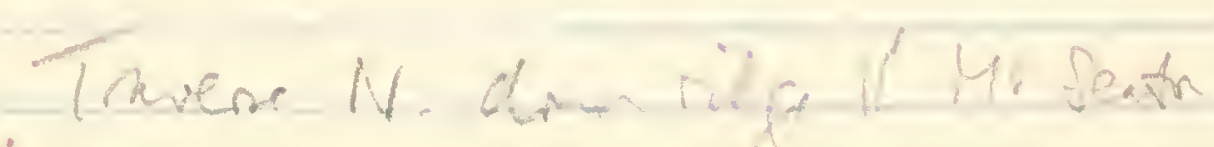

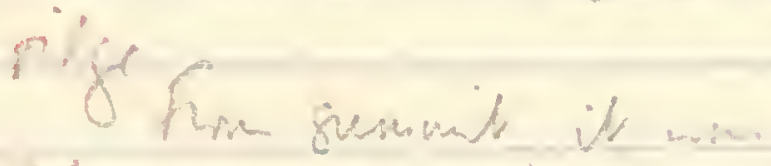

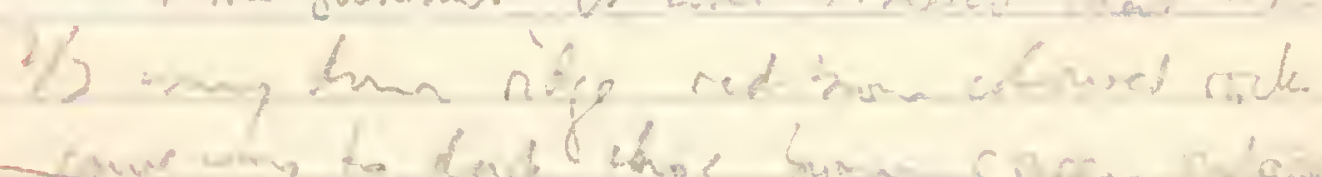

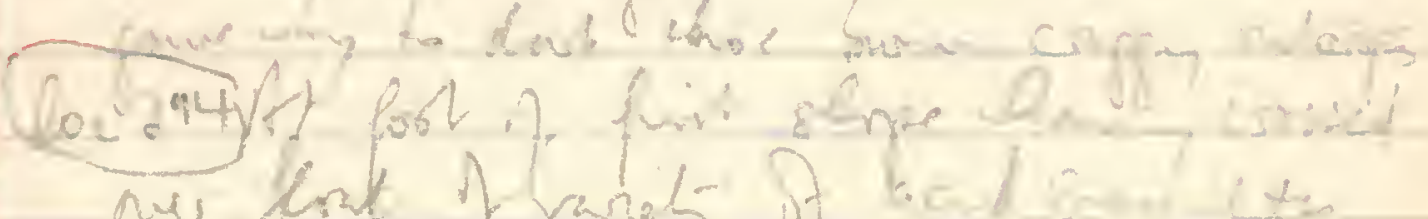
ver lont of vanets of on min the the

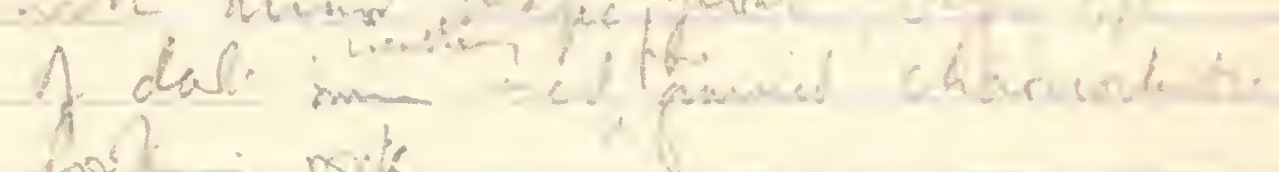

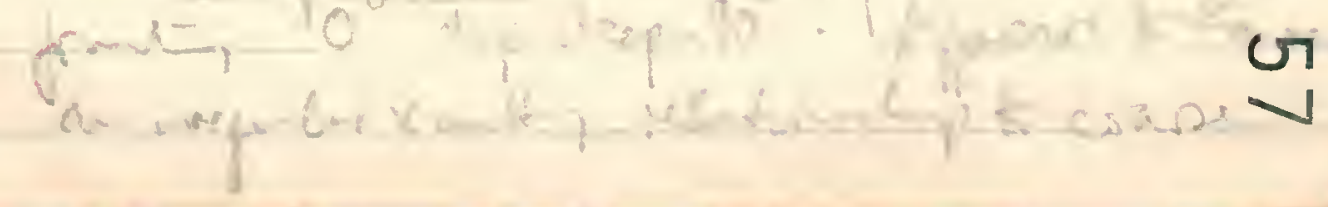


295

rus

$4418 / 1673$

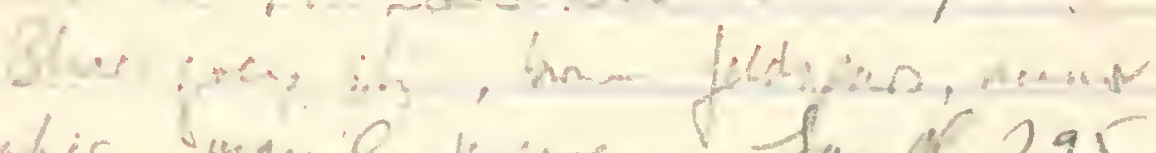

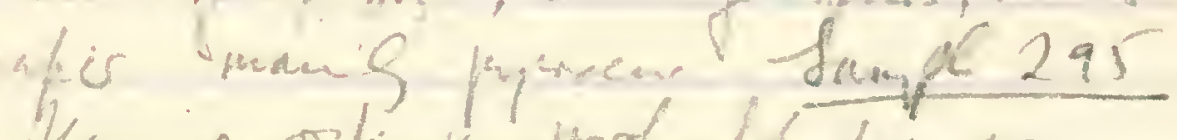

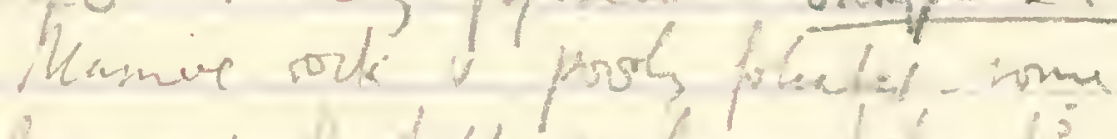

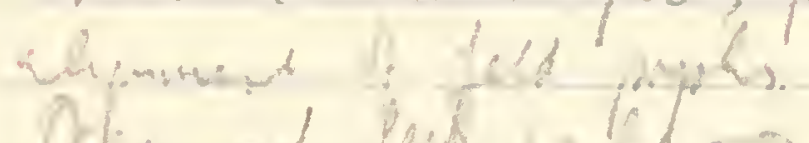

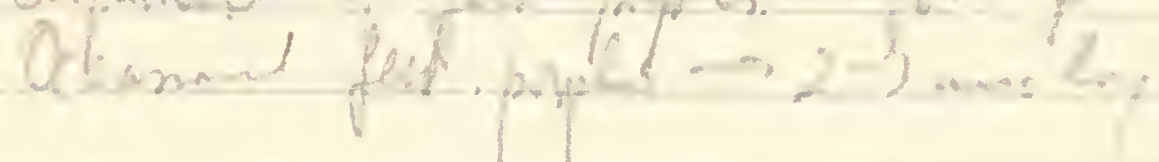

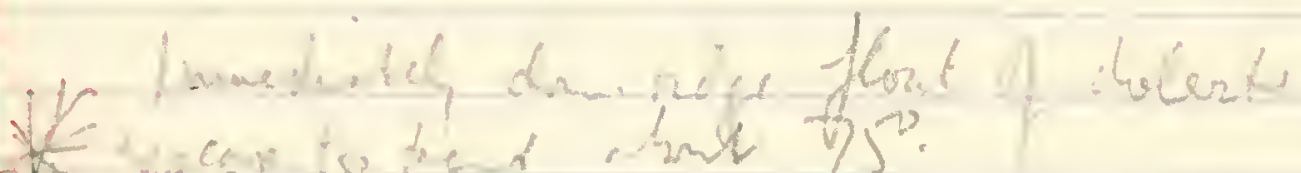

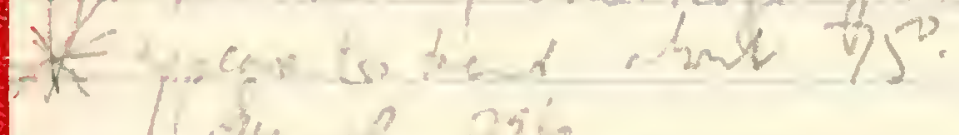

alumel 250

Filde lus an wardth of In

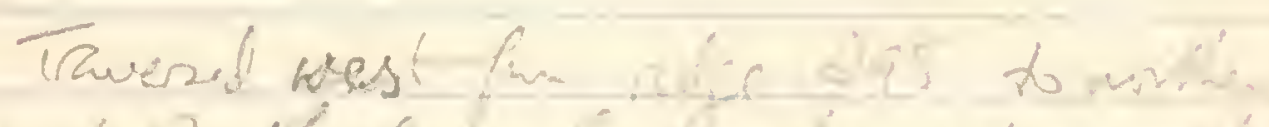

$\therefore 271$ ing 50

G 



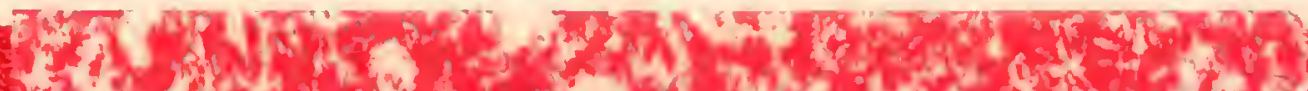

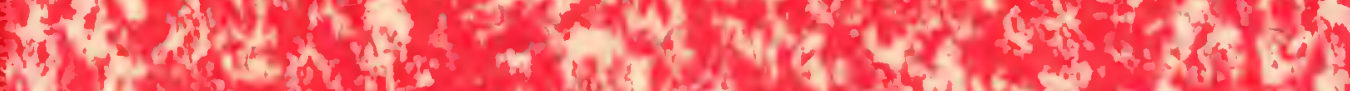

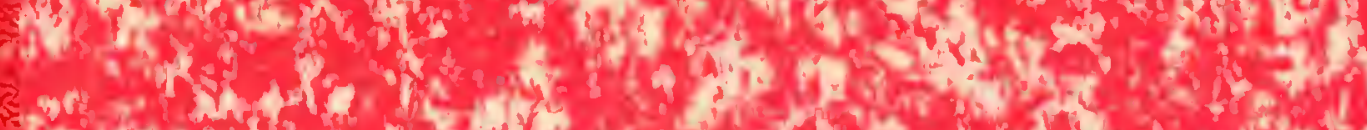

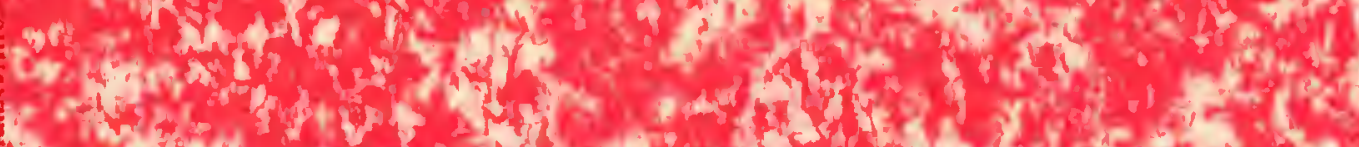

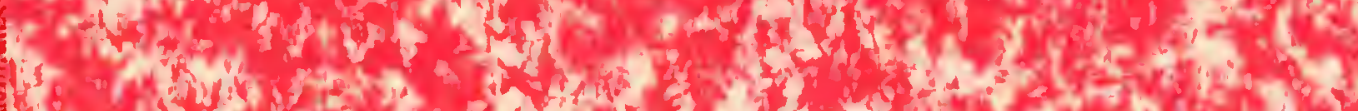

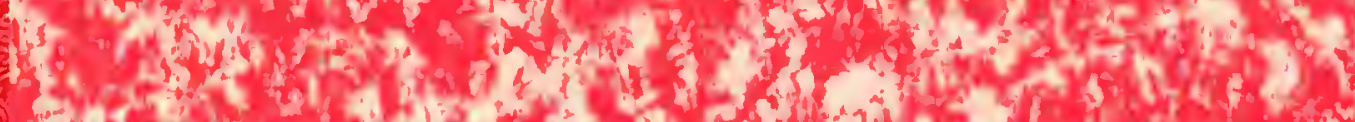

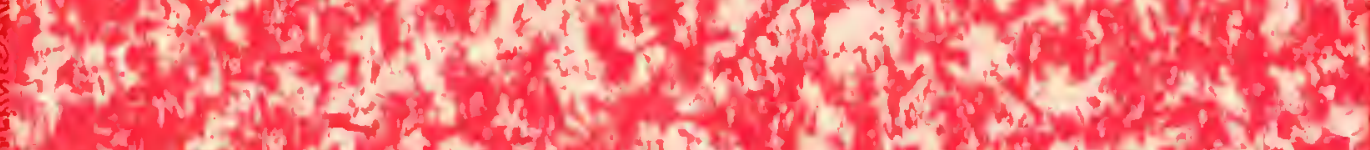

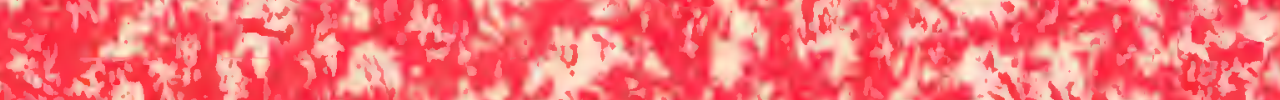

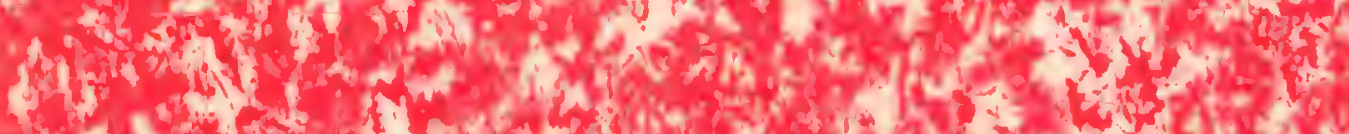

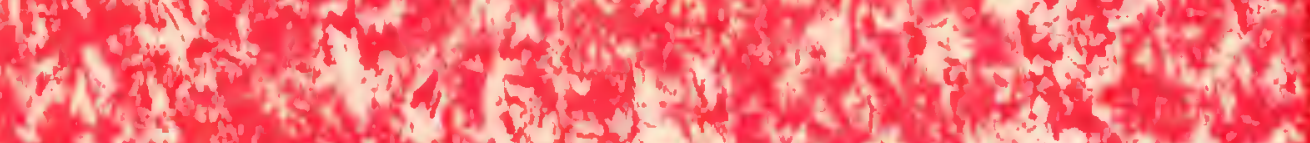

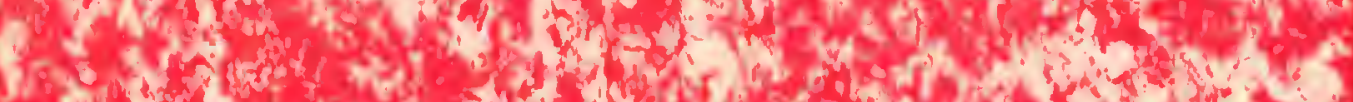
(6)

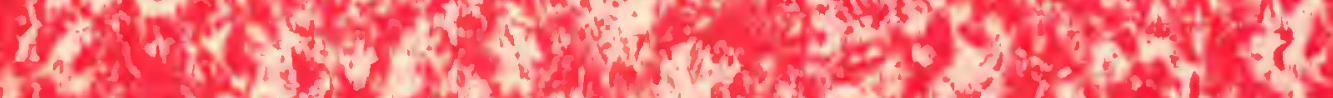

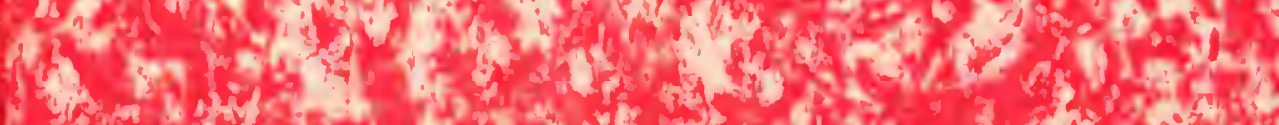

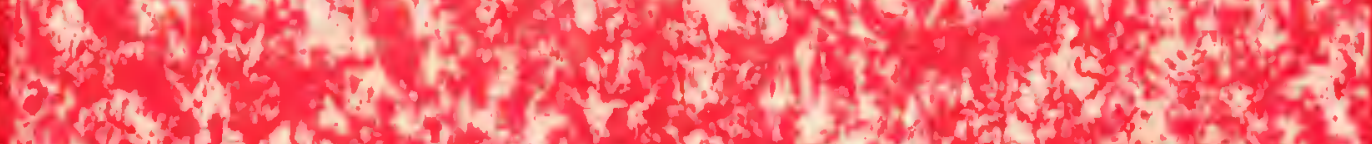

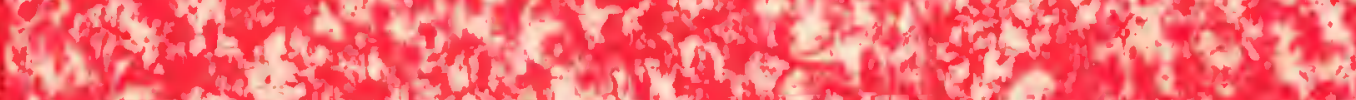

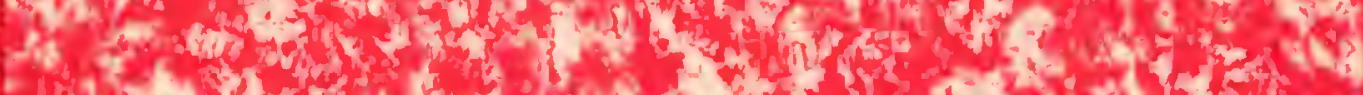

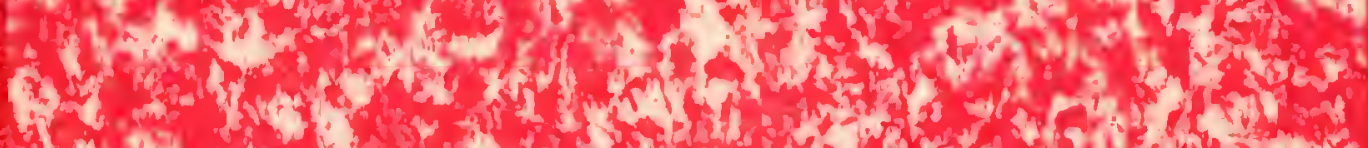

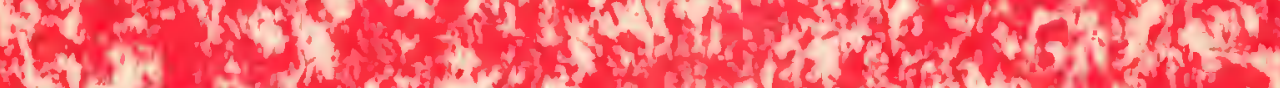

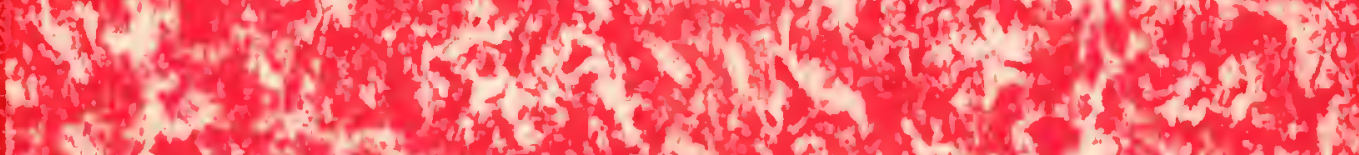

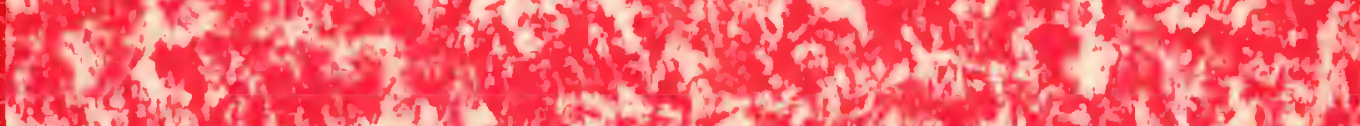

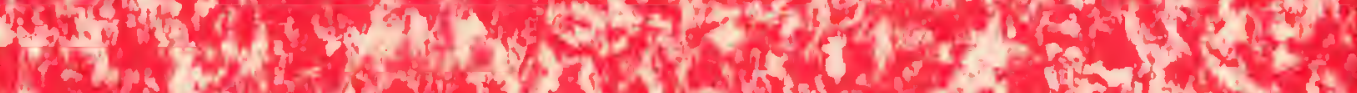

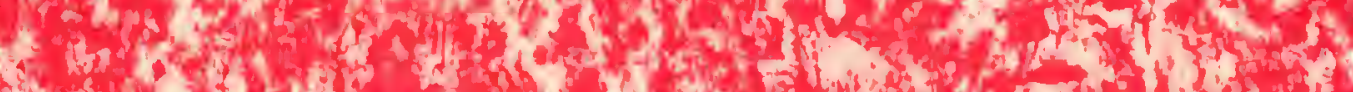

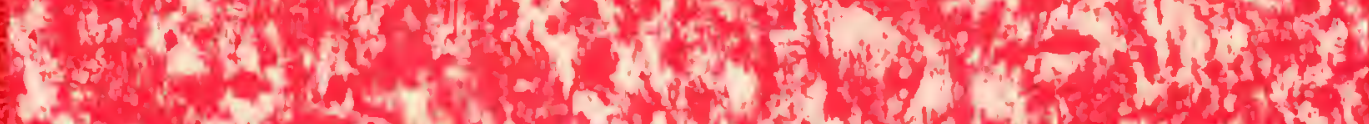

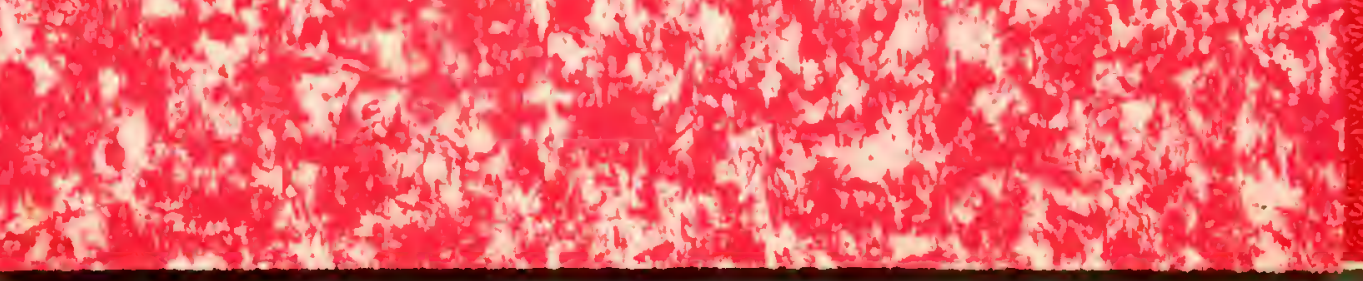



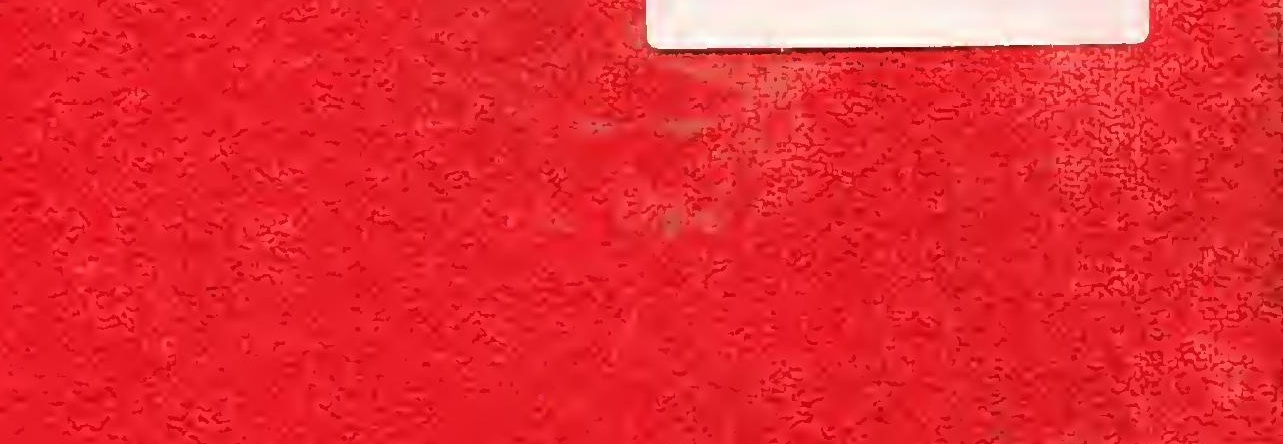

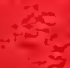

$-1 x^{2} x^{2}$
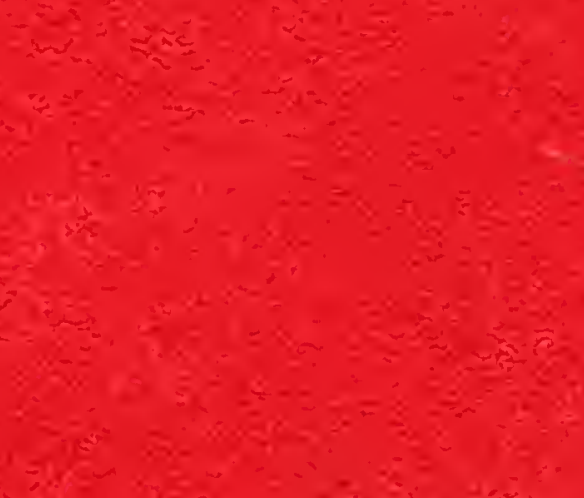

t.
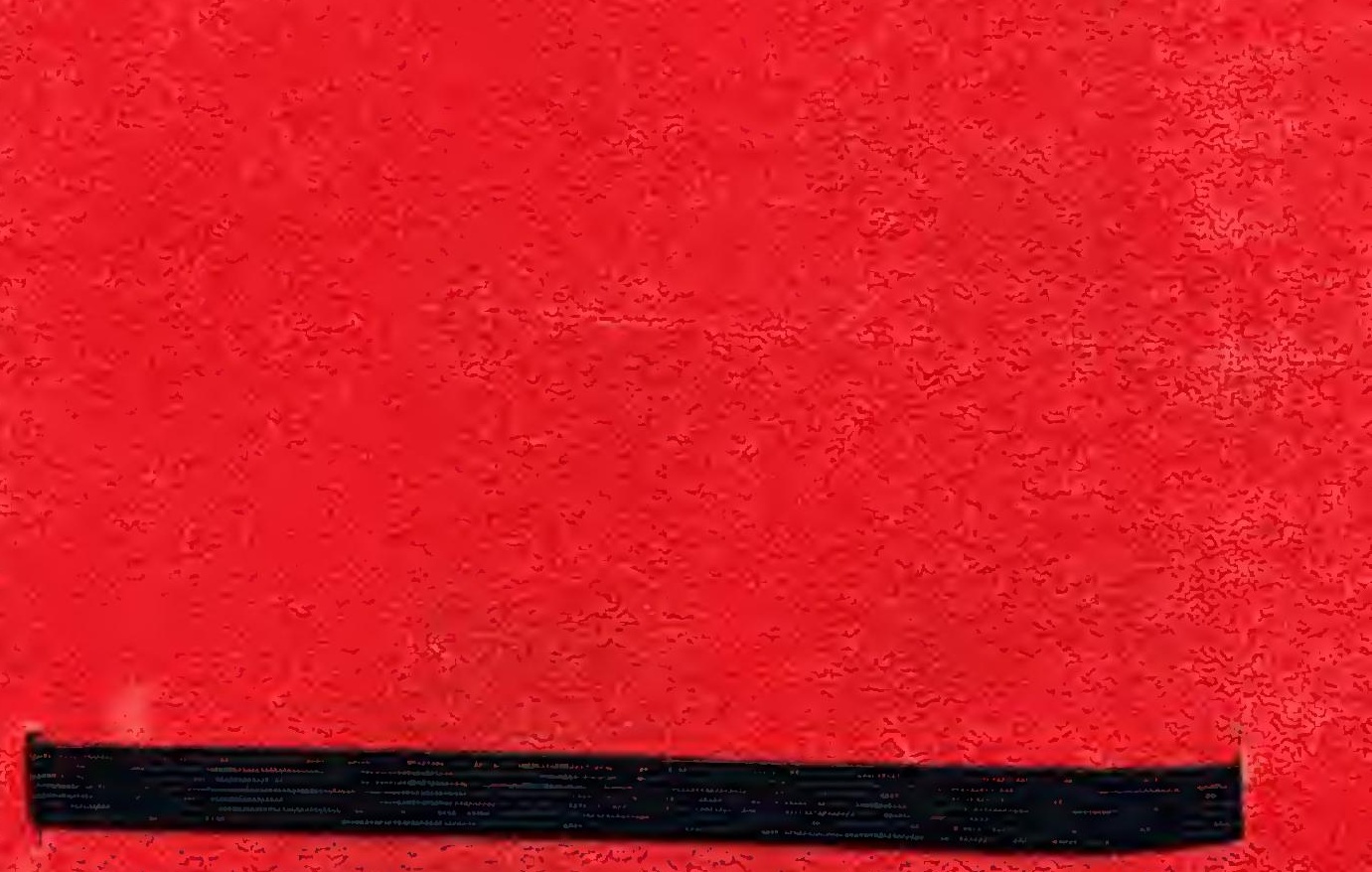

$\lim _{1}=$

$5 x^{2}-2=1-20$

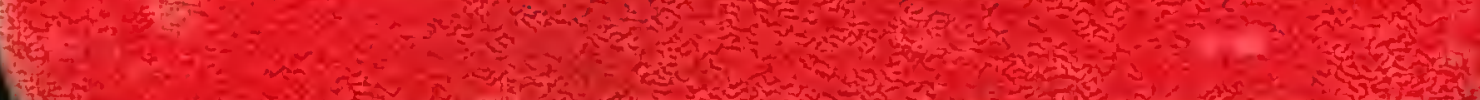
से 